\title{
An Inventory and Assessment of Archaeological Sites in the High Country of Guadalupe Mountains National Park, Texas
}

Paul R. Katz

Follow this and additional works at: https://scholarworks.sfasu.edu/ita

Part of the American Material Culture Commons, Archaeological Anthropology Commons, Environmental Studies Commons, Other American Studies Commons, Other Arts and Humanities Commons, Other History of Art, Architecture, and Archaeology Commons, and the United States History Commons

Tell us how this article helped you.

This Article is brought to you for free and open access by the Center for Regional Heritage Research at SFA ScholarWorks. It has been accepted for inclusion in Index of Texas Archaeology: Open Access Gray Literature from the Lone Star State by an authorized editor of SFA ScholarWorks. For more information, please contact cdsscholarworks@sfasu.edu. 


\section{An Inventory and Assessment of Archaeological Sites in the High Country of Guadalupe Mountains National Park, Texas}

\section{Creative Commons License}

\section{(c) (1) \&}

This work is licensed under a Creative Commons Attribution-NonCommercial 4.0 International License 


\section{AN INVENTORY AND ASSESSMENT OF ARCHAEOLOGICAL SITES IN THE HIGH COUNTRY OF GUADALUPE MOUNTAINS NATIONAL PARK, TEXAS by \\ Paul R. Katz}

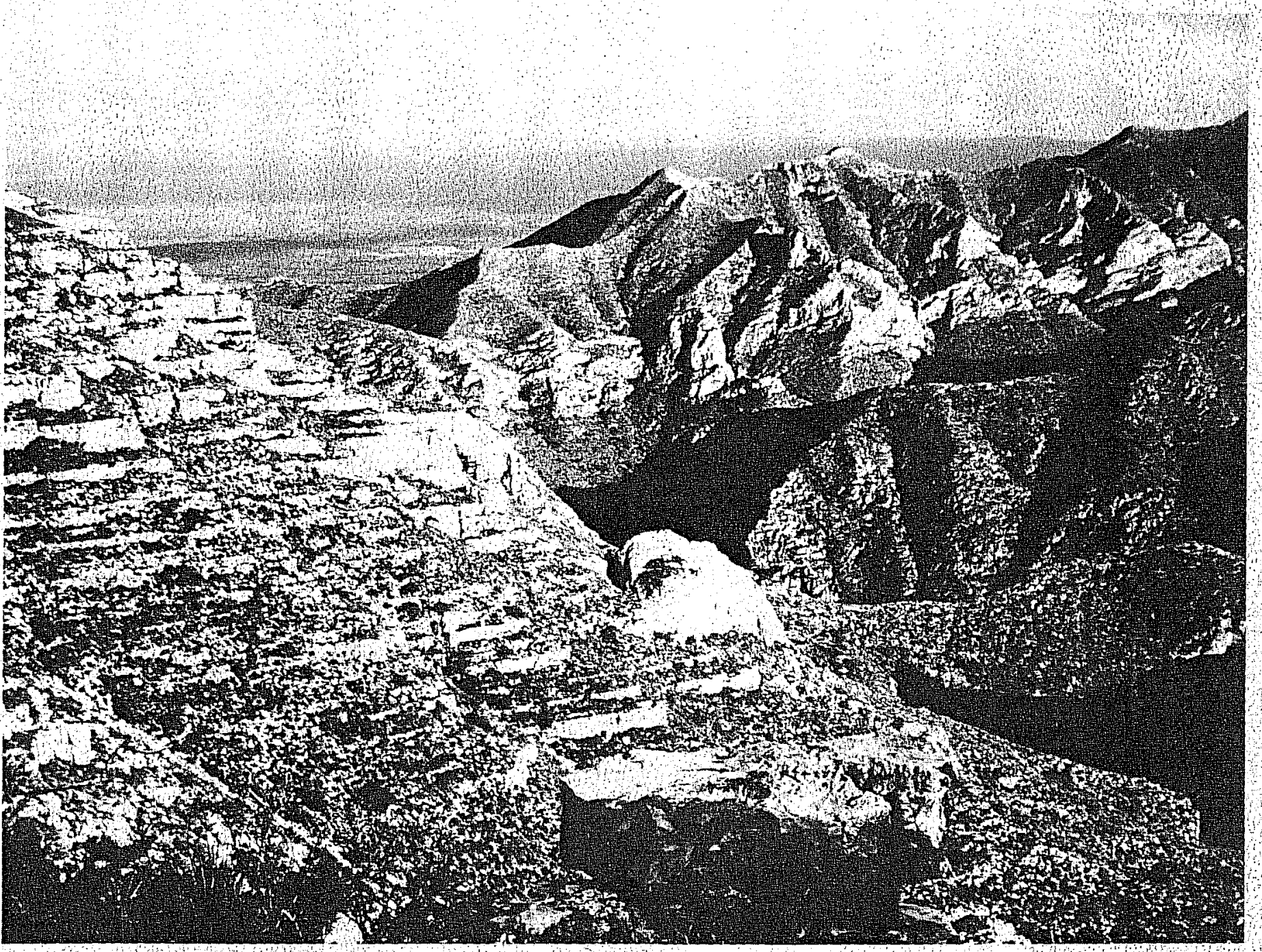

with an Appendix by Susanna R Katz

Center for Archaeological Research The University of Texas at San Antonio Archaeological Survey Report, No. 36 

, 
AN INVENTORY AND ASSESSMENT OF ARCHAEOLOGICAL SITES IN THE HIGH COUNTRY OF GUADALUPE MOUNTAINS NATIONAL PARK, TEXAS

\author{
by \\ Paul R. Katz
}

With an Appendix by Susanna R. Katz

Center for Archaeological Research The University of Texas at San Antonio

Archaeological Survey Report, No. 36

1978 
Submitted in partial fulfillment of Contract CX 702960154 between the National Park Service and the Center for Archaeological Research, The University of Texas at San Antonio. 
TABLE OF CONTENTS

Page

List of figures. . . . . . . . . . . . . . . . . . . . . i i

List of Tables... . . . . . . . . . . . . . . . . . . iv

Acknowledgments. . . . . . . . . . . . . . . . . . . . . . . . . vi

Introduction ........................... . . . 1

Aspects of the High Country. . . . . . . . . . . . . . . . . . 9

Conduct of the Survey. . . . . . . . . . . . . . . . . . . 11

Description of Archaeological Data . . . . . . . . . . . . . . 12

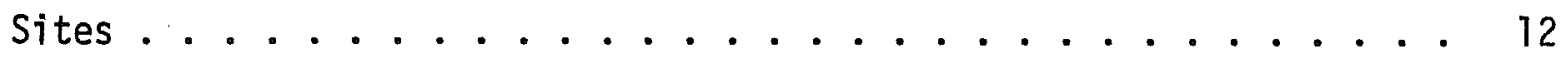

Physical Aspects.......................... 14

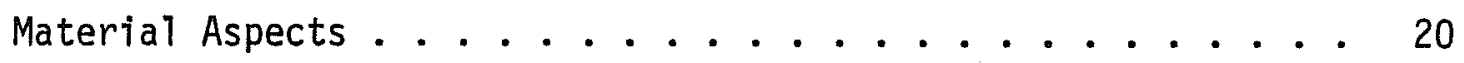

Artifacts . . . . . . . . . . . . . . . . . . 33

Collection Procedures. . . . . . . . . . . . . . 33

Descriptive Categories ................ 34

Chronologically Diagnostic Artifacts . . . . . . . . . 49

Discussion . . . . . . . . . . . . . . . . . . . . . . 54

Temporal Aspects. . . . . . . . . . . . . . . . . 54

Spatial Aspects . . . . . . . . . . . . . . . . . 57

Aspects of Subsistence and Settlement . . . . . . . . . . . . . 59

High Country Sites Considered Eligible for Listing in the National

Register of Historic Places. . . . . . . . . . . . . . . . . 62

References Cited . . . . . . . . . . . . . . . . . . . 69

Appendix I (4T CU 12). . . . . . . . . . . . . . . . . . . . . . . . . 73

Appendix II $(47$ CU 197) . . . . . . . . . . . . . . . . . . . . . . 77

Appendix III (Test Excavations at 41 CU 97) . . . . . . . . . . . . . 81 



\section{LIST OF FIGURES}

Figure

Page

1. 1976 Survey Area. . . . . . . . . . . . . . . 2

2. Location of Archaeological Sites Recorded in 1976 ....... 3

3. Location of Collecting Localities and Isolated Finds Documented

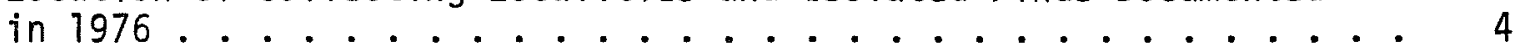

4. Topographic Situations Associated with Archaeological Sites and Material. ................. 15

5. Non-Feature Site Types. . . . . . . . . . . . . 21

6. Midden Features .................. 22

7. Locations of Recorded Archaeological Sites Over 6500 Feet

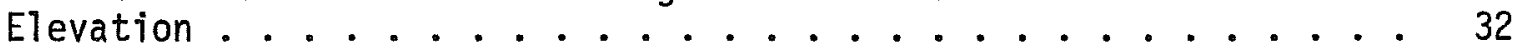

8. Chipped Stone Artifacts .............. 35

9. Chipped Stone Artifacts ............... 36

10. Chipped Stone Artifacts . . . . . . . . . . 37

11. Chipped Stone Artifacts ............... 39

12. Projectile Points Diagnostic of the Late Paleo-Indian and Early Archaic Periods................ . . 40

13. Projectile Points Diagnostic of the Middle and Late Archaic Periods.................... 41

14. Projectile Points Diagnostic of the Transitional and Ceramic Periods .................. . . 42

15. Handstones. . . . . . . . . . . . . . . . 43

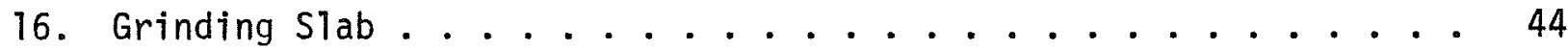

17. Representative Pottery Types Documented in the High County in $1976 \ldots . . \ldots 45$

18. Chronological Framework for the High Country. . . . . . . . . 56

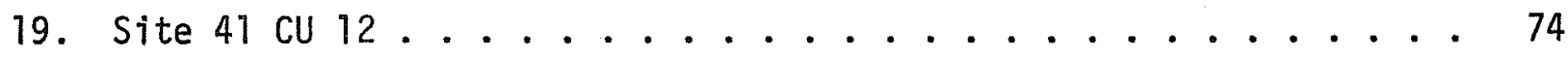

20. Selected Pictographs at $41 \mathrm{CU} 197 \ldots \ldots$. . . . . . . 78

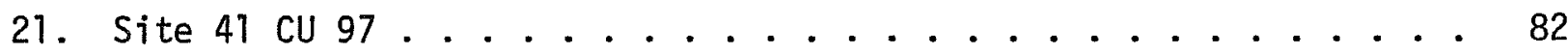



Figure

22. Selected Artifacts from 41 CU $97 \ldots \ldots 83$

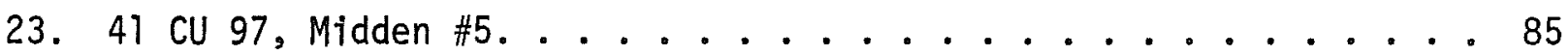

24. Selected Excavation Units at 41 CU 97. . . . . . . . . . . 86

25. Selected Excavation Profiles at 41 CU 97, Midden \#5 . . . . . 88 



\section{LIST OF TABLES}

Table

Page

1. Summary of Prehistoric Activity Loci Documented in Guadalupe Mountains National Park. . . . . . . . . . . . 5

2. Selected Data from Previously Recorded High Country (above $6500 \mathrm{ft}$ ) Sites.................... 6

3. Correlation of Newly Recorded High Country Sites and Topographic Situations ................... 16

4. Site Positions with Respect to Topographic Orientation, for Selected Topographic Situations. . . . . . . . . . 17

5. Correlation of Newly Recorded High Country Sites with Grouped

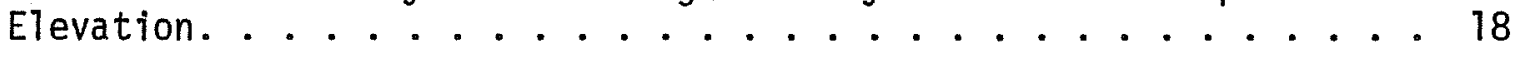

6. Correlation of Newly Recorded High Country Sites with Grouped Area........................ 19

7. Classification of Newly Recorded High Country Sites According to Feature P/A and Type............. 23

8. Classification of Newly Recorded High Country Monotypic Midden Sites.................... 24

9. Classification of Newly Recorded High Country Polytypic Midden

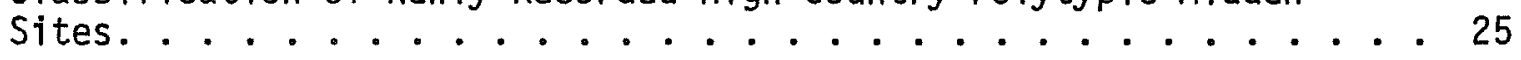

10. Newly Recorded High Country Midden Feature Dimensional Data. . . . 26

11. Summary of Midden Feature Popularity at High Country Sites . . . . 30

12. Correlation of Sites with Recovered Artifact Categories--New Sites.................... 46

13. Correlation of Sites with Recovered Artifact Categories--Previously Recorded Sites Revisited in 1976 . . . . . . . . . 47

14. Correlation of Collecting Localities and Isolated Finds with Recovered Artifact Categories. . . . . . . . . . . . . 48

15. Correlation of Projectile Point Types with Newly Recorded High Country Sites................ 50

16. Correlation of Projectile Point Types with Previously Recorded Sites Revisited in 1976. ............... 51

17. Correlation of Projectile Point Types with Collecting Localities

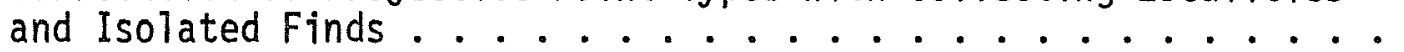



18. Sherd Classification by Site.............. 53

19. High Country Sites Considered Eligible for Listing in the National Register of Historic Places. . . . . . . . . . 64

20. Specific Site Importance Factors for Determining National Register Eligibility. ................ 65

21. Site Disturbance Variables Affecting A11 Recorded High Country

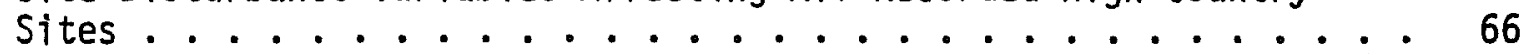

22. 41 CU 12 Feature Data ............... . 75

23. Correlation of SWRO Working Numbers and Officially Recorded Numbers for Sites and Features in Upper Dog Canyon. . . . . . . . 91 



\section{ACKNOWLEDGMENTS}

The exceptional cooperation and interest which we experienced in 1973 and 1974 in no way diminished during the 1976 season in Guadalupe Mountains National Park. We found this to be the case at all levels, from the Regional Archaeologist's office in Santa Fe to the horse patrol in the High Country. While we are certain that there are many individuals who aided in the project and of whom we are unaware, we can only acknowledge those with whom we had personal contact. These include: Ron Ice and Cal Cummings at the Southwest Regional Office in Santa Fe; Gary Ah7strand, Bob Crissman, Ron Kerbo and Phil Van Cleave at the Park Headquarters in Carlsbad; Bruce Fladmark, Roger Reisch and Sharon Cox at Guadalupe Mountains National Park; and Allan Hines, Lewis Huddy and the helitac team members, and helicopter pilot Pat Emory of the Forest Service.

We would like to belatedly extend our congratulations to Craig Dunn for escaping from his downed helicopter and thank Dick Calens of Roswell for filling in at the last minute to remove our supplies and collections from the High Country.

We are especially grateful to the Park Service personnel and Texas Tech researchers (the Elk Study team in particular) who provided information during the survey, maintained communication links with the rest of the world and generally watched over the whole operation. Harry and Anne Steed of the Upper Dog Canyon Ranger Station deserve special thanks.

We are grateful to Barbara Johns on for her purchasing efforts and to Lynn Highley, who washed and cataloged all the archaeological material. Graphic aids and photographs were produced by the UTSA Office of Instructional Services, and David Barrera deserves great credit for the long hours spent in completing the maps. The manuscript was edited, typed and proofread by staff members of the UTSA Center for Archaeological Research, Dr. Thomas R. Hester, Director.

Finally, the successful implementation of the High Country survey was due in large part to the extensive logistical preparations of Susanna R. Katz and to the impressive efforts of those latter-day "Jeremiah Johnsons," Bryan Aivazian and Paul Lukowski. 



\section{INTRODUCTION}

This report will present the results of an archaeological survey conducted in Guadalupe Mountains National Park, Texas, during the summer of 1976. The specific purpose of this survey was to compile an inventory of prehistoric resources in that portion of the Park known as the High Country, the only portion of the Park which had not yet been subjected to a comprehensive, onthe-ground archaeological reconnaissance. Data gathered during the survey were analyzed and interpreted in the light of existing cultural frameworks for the Park and surrounding region. Criteria of significance and criteria of effect were applied to all documented archaeological sites and other evidence of prehistoric activity, to assist the National Park Service in complying with the National Historic Preservation Act of 1966, the National Environmental Policy Act of 1969 and Executive Order 11593 of May 13, 1971.

Funding for the survey and subsequent analys is was provided through the Southwest Regional Office of the National Park Service, under the supervision of Regional Archaeologist Ron Ice and Contracting Officer J. I. Carpenter. The field"work was conducted by staff members of the Center for Archaeological Research at The University of Texas at San Antonio, Dr. Thomas R. Hester, Director; Paul R. and Susanna R. Katz served as Co-Field Supervisors, with Bryan Aivazian and Paul Lukowski carrying out the on-the-ground reconnaissance in the High Country.

During the period of seven weeks from June 24 through August 13, 1976, an area estimated to be $25 \%$ of the Park was surveyed for evidence of prehistoric occupation. Unlike previous surveys which sampled different portions of the Park (cf. S. Katz and P. Katz 1974:Figs.2,3), this latest reconnaissance was concentrated within a we11-defined area bounded by the eastern and western escarpments, the Texas-New Mexico Tine to the north, and the 6500-foot contour line (Fig. 1). Ninety-seven localities of prehistoric activity were recorded for the first time, comprising 65 sites, 11 collecting localities and 21 isolated finds (Figs. 2,3 ).

At the request of Regional Archaeologist Ron Ice, an additional week was spent working at lower altitudes and in different portions of the Park. The tasks included the recording of a multiple midden site (41 CU 12) adjacent to the proposed parking area at the mouth of McKittrick Canyon (Appendix I); the documentation of a pictograph site (41 CU 197) in Pine Springs Canyon (Appendix II); and the testing of several midden features at 41 CU 97, in Upper Dog Canyon, which were to be adversely affected by a proposed improvement in the access road right-of-way (Appendix III). The addition of the McKittrick and Pine Springs Canyon sites and a collecting locality recorded in Upper Dog Canyon bring the total number of activity loci recorded in 1976 to 100 (Table 1).

Other survey parties had documented sites in the High Country (Table 2), although the UTSA reconnaissance was the first to 7 imit its investigations exclusively to this portion. Previously recorded sites were thus encountered by the 1976 personnel, resulting in modest supplemental artifact collections and on occaston a slight revision of the original site survey form.

Prior to 1970, that area in west Texas now designated Guadalupe Mountains National Park saw very little archaeological investigation; and what there was 
This page has been

redacted because it

contains restricted

information. 
This page has been

redacted because it

contains restricted

information. 
This page has been

redacted because it

contains restricted

information. 


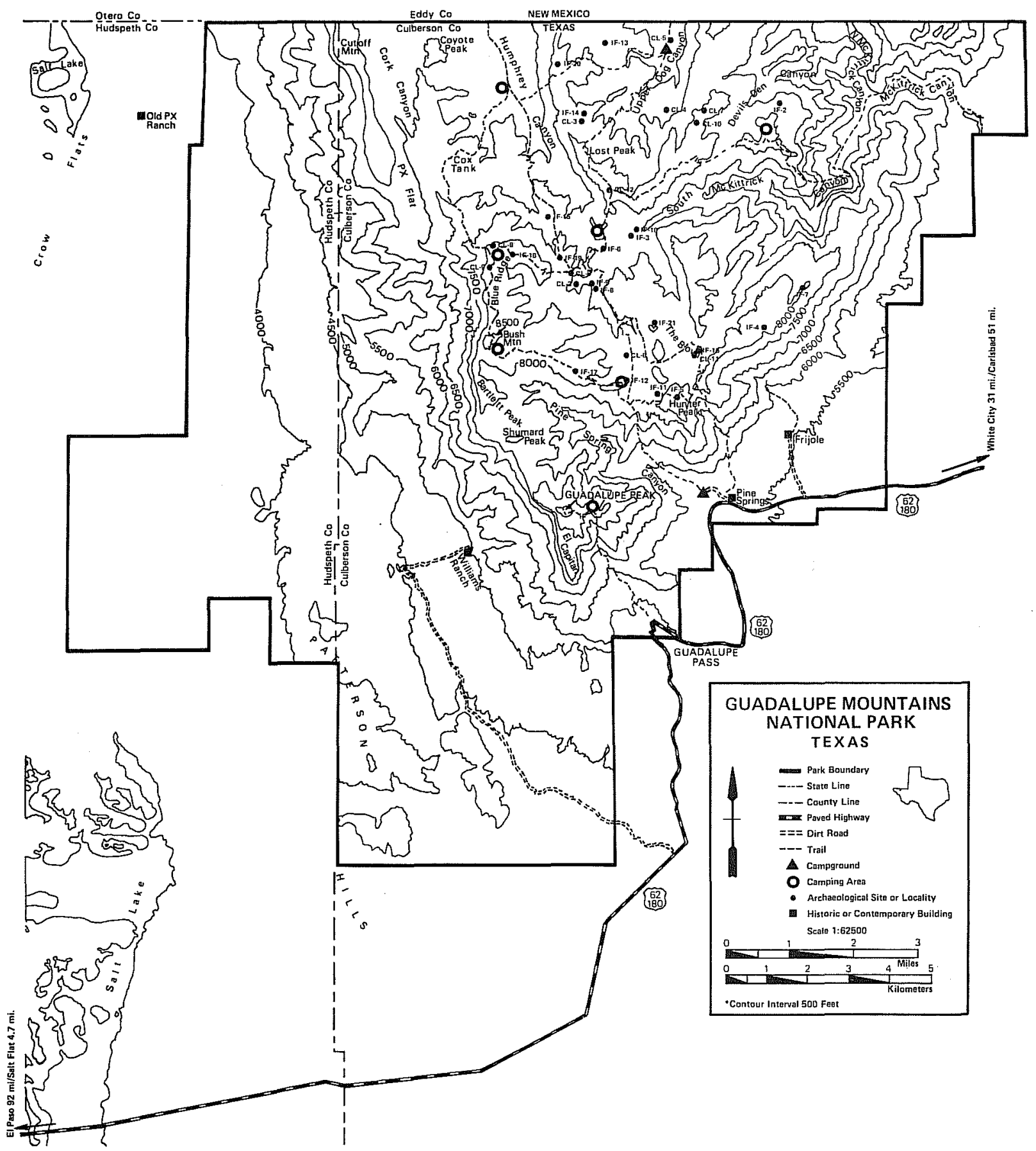

Figure 3. Location of Collecting Localities and Isolated Finds Documented in 1976. 
TABLE 1. SUMMARY OF PREHISTORIC ACTIVITY LOCI DOCUMENTED IN GUADALUPE MOUNTAINS NATIONAL PARK

\begin{tabular}{|c|c|c|c|c|c|}
\hline $\begin{array}{l}\text { Activity } \\
\text { Locus } \\
\text { Classification }\end{array}$ & Sites & $\begin{array}{l}\text { Collecting } \\
\text { Localities }\end{array}$ & $\begin{array}{l}\text { Isolated } \\
\text { Finds }\end{array}$ & TOTALS & $\begin{array}{l}\text { (\% of } \\
\text { Park } \\
\text { Surveyed })\end{array}$ \\
\hline Project & & & & & \\
\hline $\begin{array}{l}\text { Isolated } \\
\text { Recordings }\end{array}$ & 11 & & & 11 & -- \\
\hline $\begin{array}{l}\text { Texas } \\
\text { Archeological } \\
\text { Society (1970) }\end{array}$ & 139 & 19 & & 158 & (35) \\
\hline $\begin{array}{l}\text { Texas Tech } \\
\text { University } \\
(1973)\end{array}$ & 68 & 15 & & 83 & (35) \\
\hline $\begin{array}{l}\text { Texas Tech } \\
\text { University } \\
\text { Field School } \\
(1974)\end{array}$ & 14 & & & 14 & ( 5$)$ \\
\hline $\begin{array}{l}\text { University of } \\
\text { Texas at } \\
\text { San Antonio } \\
(1976)\end{array}$ & 67 & 12 & 21 & 100 & (25) \\
\hline TOTALS & 299 & 46 & 21 & 366 & $(100)$ \\
\hline
\end{tabular}


TABLE 2. SELECTED DATA FROM PREVIOUSLY RECORDED HIGH COUNTRY (ABOVE 6500 FT) SITES

\begin{tabular}{|c|c|c|c|c|c|c|c|c|c|c|}
\hline $\begin{array}{l}\text { Data } \\
\text { Categories } \\
\end{array}$ & 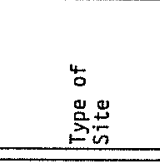 & 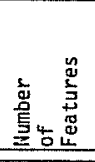 & 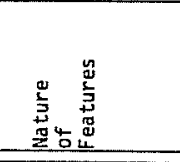 & 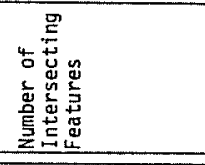 & 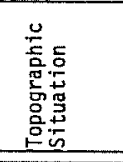 & 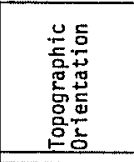 & 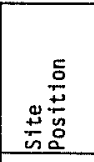 & 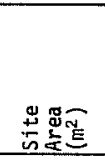 & 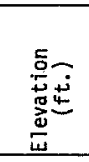 & 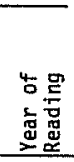 \\
\hline \multicolumn{11}{|l|}{ Site No. } \\
\hline $41 \mathrm{CU} \quad 23$ & Rockshel ter & 0 & $N / A$ & $\mathrm{H} / \mathrm{A}$ & $\begin{array}{l}\text { Vertical } \\
\text { Face }\end{array}$ & $\begin{array}{l}N-S \\
E-W\end{array}$ & $\underset{\mathrm{SE}}{\text { Facing }}$ & 1524 & 7000 & 1970 \\
\hline $41 \mathrm{CU} \quad 24$ & Rockshelter & 0 & $N / A$ & IN/A & $\begin{array}{l}\text { Vertical } \\
\text { Face }\end{array}$ & $\begin{array}{l}\text { NE- } \\
\text { SW }\end{array}$ & NE & 892 & 7000 & 1970 \\
\hline $41 \mathrm{CU} \quad 80$ & Midden & 3 & $\begin{array}{l}2 \text { Rings; } \\
1 \text { Mound }\end{array}$ & $\begin{array}{l}\text { 2; } 1 \text { Ring } \\
\text { \& I Mound }\end{array}$ & Saddle & $\begin{array}{l}\text { NW- } \\
S E\end{array}$ & NE & 18,288 & 7850 & 1970 \\
\hline 41 CU 94 & Midden & 1 & Ring & $N / A$ & $\begin{array}{l}\text { Stream } \\
\text { Terrace }\end{array}$ & $\mathrm{N}-\mathrm{S}$ & $w$ & 54 & 6750 & 1970 \\
\hline $41 \mathrm{CU} 139$ & Midden & 1 & Mound & $N / A$ & Saddle & $E-W$ & N & 625 & 7150 & 1973 \\
\hline 41 CU 140 & Midden & 5 & $\begin{array}{l}4 \text { Rings; } \\
\text { I Mound }\end{array}$ & $\begin{array}{l}4 ; 2 \text { sets } \\
\text { of paired rings }\end{array}$ & Saddle & $\mathbb{H}-\mathrm{S}$ & -- & 8382 & 7800 & 1973 \\
\hline $41 \mathrm{CU} 147$ & Midden & 1 & Ring & $N / A$ & $\begin{array}{l}\text { Stream } \\
\text { Terrace }\end{array}$ & $E-W$ & N & 724 & 6600 & 1973 \\
\hline $41 \mathrm{CU} 161$ & $\begin{array}{l}\text { Lithic } \\
\text { Scatter }\end{array}$ & 0 & $\mathrm{~N} / \mathrm{A}$ & $N / A$ & $\begin{array}{l}\text { Stream } \\
\text { Terrace }\end{array}$ & $N-5$ & $W$ & 2394 & 7850 & 1973 \\
\hline 41 CU 169 & Midden & 4 & $\begin{array}{l}2 \text { Rings; } 1 \\
\text { Mound; } 1 \\
\text { Indeterminate }\end{array}$ & 0 & Saddle & N-S & -- & 3741 & 6950 & 1973 \\
\hline $41 \mathrm{CU} 175$ & Midden & 3 & 3 Rings & 3 & $\begin{array}{l}\text { Kidge } \\
\text { Crest }\end{array}$ & $\mathrm{H}-\mathrm{S}$ & -- & 13,716 & 7550 & 1973 \\
\hline 47 CU 176 & Midden & 1 & Ring & $N / A$ & Saddle & $\mathrm{H}-\mathrm{S}$ & -- & 383 & 7825 & 1973 \\
\hline $41 \mathrm{CU} 177$ & Midden & 1 & Ring & $N / A$ & Saddle & $E-W$ & --- & 599 & 8000 & 1973 \\
\hline $41 \mathrm{CU} 180$ & Midden & 1 & Indeterminate & $N / A$ & $\begin{array}{l}\text { Summit } \\
\text { Terrace }\end{array}$ & $\begin{array}{c}\text { South side } \\
\text { of } \\
\text { Sumit }\end{array}$ & & 5386 & 6550 & 1973 \\
\hline $47 \mathrm{CU} 181$ & $\begin{array}{l}\text { Lithic } \\
\text { Scatter }\end{array}$ & 0 & $N / A$ & $N / A$ & $\begin{array}{l}\text { Stream } \\
\text { Terrace }\end{array}$ & N-S & -- & 114 & 6750 & 1973 \\
\hline 41 CU 182 & Midden & 1 & Ring & $N / A$ & Saddle & $E-W$ & - & 96 & 8200 & 1973 \\
\hline $47 \mathrm{CU} 183$ & Midden & 1 & Ring & $N / A$ & $\begin{array}{l}\text { Ridge } \\
\text { Crest }\end{array}$ & $E-W$ & -- & 54 & 6600 & 1973 \\
\hline $41 \mathrm{CU} 184$ & Midden & 1 & Indeterminate & $N / A$ & Sadd?e & $E-W$ & -- & 1350 & 7950 & 1974 \\
\hline $41 \mathrm{CU} 185$ & Midden & 3 & $\begin{array}{l}2 \text { Rings; } \\
1 \text { Mound }\end{array}$ & $\begin{array}{l}\text { 2: } \\
2 \text { Rings }\end{array}$ & $\begin{array}{l}\text { Stream } \\
\text { Terrace }\end{array}$ & $\mathrm{N}-\mathrm{S}$ & W & 3750 & 7580 & 1974 \\
\hline $41 \mathrm{CU} 186$ & $\begin{array}{l}\text { Lithic } \\
\text { Scatter }\end{array}$ & 0 & N/A & $N / A$ & $\begin{array}{l}\text { Streamı } \\
\text { Terrace }\end{array}$ & $N-5$ & W & 491 & 7500 & 1974 \\
\hline $\begin{array}{l}\text { High Cave } \\
\text { (Upper Sloth } \\
\text { Cave; GUMO } \\
\text { Cave-08) }\end{array}$ & Cave & 1 & Ring & $N / A$ & $\begin{array}{l}\text { Vertical } \\
\text { Face }\end{array}$ & $E-W$ & $\begin{array}{l}\text { Facing } \\
\mathrm{S}\end{array}$ & 1577 & 6750 & $\begin{array}{l}1931 ; \\
1974\end{array}$ \\
\hline
\end{tabular}


focused on the excavation of cave sites. Henry P. Mera (1938) excavated a midden ring inside a cave on the western escarpment in 1931, known then as High Cave and now as Upper Sloth Cave or GUMO Cave-08 (Table 2). The focus of Mera's investigation, however, was on the eastern slopes of the Guadalupe Mountains, in southeastern New Mexico. Also as part of a larger, more regionally oriented project, Edgar B. Howard directed stratigraphic excavations in Williams Cave during the summers of 1934 and 1935. Williams Cave (41 CU 16) is located in the southern portion of the Park. The report of investigations was published by Mary Y. Ayer (1936); most of the recovered materials are on display in the Carlsbad City Museum. Pratt Cave, in the northeastern portion of the Park, was initially tested in 1961 and then more thoroughly excavated in 1965 by National Park Service personnel (Schroeder 1965). First designated GUMO A-1, Pratt Cave is now officially recorded as GUMO Cave-02 and archaeological site 41 CU 196.

The first organized archaeological survey in the Park was sponsored by the Texas Archeological Society. About 200 Society members spent 10 days in June 1970 learning survey methods and techniques while providing the first coherent body of data concerning the prehistoric occupation of the Park. The TAS survey recorded 139 new sites, visited 11 previously recorded sites and documented 19 collecting localities. A brief report on their activities and data was published by Harry J. Shafer (1970), with more detailed analyses of ceramics (Phelps 1974) and rock art (Clark 1974) appearing several years later.

of the 11 sites recorded prior to the TAS survey, a number of them were located and documented by Eugene Anderson during many years of independent surveying. Of particular note are several pictograph caves, including Smith (41 CU 15) and Apache Mask (41 CU 24), which are discussed in detail by Clark (1974).

Although the TAS survey was conducted one year before the signing of Executive Order 11593, it is considered as the first of a series of inventory projects which concluded with the present study. Mention should also be made of the historical component of the overall cultural resources inventory, i.e., the survey conducted by William Griggs (1973) of Texas Tech University.

The prehistoric inventory was taken up again in 1973 by Texas Tech University. First, Dr. William J. Mayer-Oakes spent several days in June in the northern portion of the Park. This was followed by a 3 1/2-month survey carried out by Paul R. and Susanna R. Katz during the late fall and early winter (S. Katz and P. Katz 1974). The Katzes focused on those portions of the Park below 6500 feet elevation which had not been surveyed by the TAS, although 12 sites were recorded in what is here being defined as the High Country portion (Table 2).

The Katzes returned to the Park the following summer to conduct an archaeological field school through the Department of Anthropology at Texas Tech University. Excavations were concentrated at four sites in the mouth of Pine Springs Canyon (P. Katz and S. Katz 1974, 1975), but some new sites were recorded, including three in the High Country (Table 2).

Prior to the 1976 UTSA survey, 232 sites had been recorded in the Park, reflecting an estimated $75 \%$ coverage of the Park (Table 1). Twenty of these sites were situated in the High Country, less than $9 \%$ of the documented total (Table 2). The remaining $25 \%$ of the Park, all High Country, yielded 65 new 
sites; High Country sites (85) now make up over $28 \%$ of the Park's total number of sites (299). It can be seen that the High Country's share of recorded sites and its estimated percentage of total Park acreage (28\%) are equivalent, suggesting that there are neither more nor fewer sites in this portion of the Park than might be located in other portions. This is a point that will be made in other contexts throughout the report--that despite its seeming isolation and inaccessibility for reasons of topography and elevation, the High Country cultural manifestations seem no different than those documented in every other portion of the Park.

There is one final point which seems appropriate to mention in this introductory section. It concerns the fact that the field directors of the current project also participated in one of the earlier inventory phases and authored the resulting report ( $S$. Katz and $\mathrm{P}$. Katz 1974). In one sense, therefore, we look upon the present report as a sequel to, or continuation of, the former. In this regard we were understandably hesitant to institute any radical departures from the classificatory schemes and interpretive framework employed in our first report. On the other hand, several factors have been introduced which make certain changes both necessary and desirable.

First, there have been at least half a dozen monographs written in the last two years which bear directly or indirectly on the prehistory of the Guadalupe Mountains. While none of these researchers has had the Park as a focus of study, taken together the locales represented by these reports completely encircle the Guadalupe Mountains locality of Texas: the lower Salt Basin southwest of the Park (Banks 1975); Hueco Bolson to the west (Whalen 1977); the eastern slopes of the Guadalupe Mountains immediately northeast (Applegarth 1976); the locality slightly farther northeast between Carlsbad and Artesia (Henderson 1976); a locality just south of White's City (Beckett 1977); and the adjacent region of central Texas (Weir 1976a, 1976b).

In addition, several of these authors have read our previous report and have referred to it in their discussions (e.g. Henderson 1976 and Weir 1976a refer to our midden classificatory scheme). Banks (1975) spends several pages summarizing and commenting on many of our interpretations and procedures. The net effect of this recent archaeological work in the region and the use to which the 1973 data has been put by several of these researchers has been to retain the site classificatory scheme and refine the chronological framework.

Finally, the writer applauds Henderson's (1976:47) stated goal:

Recent work in the Middle Pecos Region has recognized several kinds of sites in the area (Jelinek 1967; S. Katz and P. Katz 1974; Shafer 1970); however, in these cases 1ittle information has been available to explain the differences recognized. The classification here is an attempt to place descriptive classification on an explanatory level.

It is the writer's firm belief that data currently available at prehistoric sites in Guadalupe Mountains National Park has the potential of explaining a great many aspects of aboriginal life and times. But as yet that potential has not been realized. The inventory surveys conducted in 1970, 1973 and 1976 were commensurate with the level of general Park development; they provide a "feeling" 
for this particular resource and a data base on which to make sound management decisions. As the Park moves into focused developmental phases, so too will the archaeology be oriented toward specific site excavations, which will in turn lead to the solution of certain problems or the "explanation" of certain phenomena.

No one is more disappointed than the writer that more interpretations could not have been made, interpretations relating to different types of sites, site functions, specific subsistence activities or environmental change, for instance. It is the writer's opinion that the appropriate data for these interpretations can only be generated by controlled excavation. To attempt to make them in any detail based on data gathered during an inventory survey would be an academic exercise at best, as well as running counter to the phased approach to archaeological investigation and interpretation currently in effect at all levels of contract and conservation archaeology.

\section{ASPECTS OF THE HIGH COUNTRY}

The High Country portion of Guadalupe Mountains National Park is much easier to outline than it is to describe. It can be encompassed by a scalene triangle, whose base extends 10.5 miles from El Capitan to the northeast corner of the Park along the 6500-foot contour. Another side, $8.75 \mathrm{miles}$ long, connects El Capitan and Coyote Peak along the 6500-foot contour; the third side is formed by the 8.5 miles of Texas-New Mexico line from Coyote Peak to the northeast corner of the Park (Fig. 1). Calculation of the area subsumed by the above triangle results in a figure of 34 square miles, or 21,760 acres; this represents $28 \%$ of the Park's 77,500 total acreage.

The estimate of 25\% of the Park area that was surveyed in 1976 was based on the fact that small portions of the High Country had been examined previously. These include Guadalupe Peak and the northwestern portion of the High Country between Cox Tank, Coyote Peak and Cutoff Mountain. The dashed line in Figure 1 indicates the approximate boundary of the survey area.

Setting the vertical boundary of the High Country along the 6500-foot contour was based on both cultural and natural factors. This particular contour lies approximately midway on the eastern and western escarpments. As these remnants of a Permian limestone uplift are quite precipitous, the escarpments are characterized by the lowest site density of any topographic situation in the Park. This is not to say that sites were not recorded on the escarpments; all but one, however, are shelter or cave sites. The near vertical faces did seem to provide the only impediment to open site activities which characterize every other portion of the Park.

Employing natural criteria, there seems to be general agreement that 6500 feet elevation is the boundary between the Upper Sonoran and Transition life zones (Bailey 1913; Shelford 1963). As the Transition/Canadian Tife zone boundary is generally considered to be at 8000 feet elevation, the survey area cannot be limited to the former but must be associated with both the Transition and Canadian zones according to this classification scheme. The interested reader is referred to Bailey (1913) for extensive lists of the flora and fauna associated with each of these zones. 
Employing the life zone classification system to the High Country based on elevation alone becomes somewhat arbitrary when the topography is considered. With bare ridges overlooking steep-sided, narrow valleys, there are a variety of sunlight, soil and shelter conditions which result in an impressive inventory of flora and fauna. Although related to elevation, the classification system based on vegetative types seems an appropriate means of distinguishing the area in question, as the woodland and forest types are limited primarily to the High Country (National Park Service 1975, 1976; Warnock 1974). The woodland type of vegetation, "which consists primarily of oaks, junipers, pinon pines and similar small trees" (Warnock 1974:xviii), is concentrated primarily in the PX Flat-Cox Tank area and the Upper Dog Canyon-Devils Den Canyon area (Fig. 1).

The highest type of plant community in the Guadalupe Mountains is known as the forest formation of late Pleistocene. It is well represented in the moist canyon bottoms of north and south McKittrick and the tops of the mountains where it sometimes extends down the slopes to the north side of the Guadalupe Mountains National Park. The most important plants of the forest formation are: Bigtooth maple; Ponderosa pine; Limber pine; Quaking aspen; Douglas fir (Warnock 1974:xix).

The forest vegetation is concentrated in an area extending from the Blue Ridge eastward to the eastern escarpment and from Guadalupe Park north to Lost Peak (Fig. 1).

The grassland vegetative type effectively bounds the High Country by forming a "V" along the eastern and western escarpments, meeting below Guadalupe Peak. A wedge of grassland type does occur in the High Country, having its base along the Texas-New Mexico line between Humphrey and Upper Dog Canyons and narrow fingers penetrating as far south as the heads of both Devils Den and South McKittrick Canyons (Fig. 1). The most important grasses include Andropogon sp., Boutelous sp., Muhlenbergia sp., Panicum sp. and Stipa sp., with small trees and shrubs scattered throughout the grassland (Warnock 1974:xviii). The interdigitation of grassland with both woodland and forest in the High Country is particularly significant for cultural interpretations because of the inclusion of the fiber plants among the grassland vegetative types: Agave sp., Dasylirion leiophyllum, Nolina sp. and Yucca sp. (Warnock 1974:xviii). The association of these fiber plants and burned rock features such as midden rings has been well documented ethnographically and generally assumed archaeologically, representing a subsistence pattern basic to the Late Prehistoric and Historic Southwest. The identification of midden rings at $39(46 \%)$ of the High Country sites seems to establish the presence of this subsistence pattern in the highest altitudes of the Park. In fact, the highest site recorded to date in the Park--41 CU 182 at 8200 feet--is a midden ring below Guadalupe Peak.

There does seem to be a relationship between elevation and climate in the Park, in that the High Country will often vary climatically from the lower elevations in fairly predictable ways. Temperatures, for example, tend to average $10^{\circ} \mathrm{F}$ lower at higher elevations; when the winds are high in Guadalupe Pass (60 to 80 miles per hour are not unusual), they will be even higher in the High Country. Moisture tends to be significantly greater higher up in the Park, partly due to winter snow and spring snowmelt and partly because clouds often pile up around the highest peaks and limit rainfall only to this area. 
The summer months of June, July and August are the wettest, July accounting for an average of four inches of rainfall out of a yearly average of 22 inches. On the other hand, the snow has melted, and the high winds of spring have abated. It is the best time of year to conduct prolonged work in the High Country. While it is true that 10 days were lost due to inclement weather during the summer of 1976, the fact remains that the survey was completed as originally planned.

\section{CONDUCT OF THE SURVEY}

Access to that portion of the High Country in which the survey was conducted can only be gained by foot or horse, and horse traffic is severely restricted even for Park personnel. At one time it was possible to drive a jeep from Humphrey Canyon up along the Guadalupe Ridge and into the Bowl, but this "road" was allowed to fall into disrepair with the cessation of ranching operations. A very primitive road still exists from Upper Dog Canyon to Coyote Peak, passable in dry weather with a four-wheel drive vehicle.

Given the amount of territory to be covered within the allotted seven weeks (about 30 sections or 20,000 acres), it was deemed impractical to expect the field party to constantly climb up and down the escarpment every few days for supplies of food and water. Permission was obtained from the National Park Service to establish a base camp at some centrally located point in the High Country, to be supplied only once by helicopter at the initiation of the survey period. For this we are immensely gratefur, and this one fact accounts in large part for the successful completion of the project.

The helicopter phase of the operation was not without its difficulties, however. Weight restrictions per load and the cost per hour of flying time had to be balanced against the desire to ferry up seven weeks of food, water and equipment. The only mishap during this phase of the project was the loss of all the water, when two 55-gallon drums fel1 into Upper Dog Canyon after the cargo hook malfunctioned. The Forest Service, which was providing the helicopter, immediately replaced the water, but the remains of the drums ended up in Upper Dog Canyon and never saw service in the High Country.

The helicopter was also limited in its landing possibilities, and the base campsite had to be selected from the air on the first flight. The site was neither level nor as centrally located as originally planned, but both conditions were considered no more than inconveniences by the field party. The campsite was located at the junction of the Lost Peak, Mescalero and McKittrick Trails (between sites 41 CU 205, 217 and 225 on Fig. 2).

The survey design was predicated on a $100 \%$ coverage of that area whose horizontal and vertical boundaries were defined above, with the exception of any place which the survey party determined could not be reached safely on foot. No technical climbing gear was provided, nor would the National Park Service have permitted any, due to the soft and crumbly nature of the rock. Given this assumption that all territory which could be covered would be covered, no formal subdivision of the survey area was instituted.

Beginning with the three-way trail junction near camp as a focal point, the field party first surveyed the entire trail system in the High Country. This 
initial phase served the dual purposes of acclimating the party to the terrain and $i$ ts various hazards, as well as toughening them in preparation for the more rigorous phases to follow. Each major ridge system was then surveyed (e.g., Blue, Guadalupe), beginning with the primary ridge and following up with ail the spur ridges. Then the canyons were investigated (e.g., Trail, South Mckittrick), including the ridge arms and their spurs which bounded each canyon. Specific trips were made to unique areas, such as the Bowl in the southern portion of the High Country, and to the more remote portions, such as Wilderness Ridge in the northeast corner of the survey area, and to the top of the eastern escarpment (Fig. 1). The more distant portions from the base camp necessitated bivouacs of several days duration; access to Wilderness Ridge could only be accomplished via the Lincoln National Forest in New Mexico. The members of the survey party left the High Country infrequently and at their own discretion, usually only after a particularly rigorous section was completed or if an extended period of inclement weather was expected.

Another element of the survey design was concerned with the consistency of data recording. In order to maximize the comparability of data gathered, the same site survey forms and site classificatory scheme were employed in 1976 as were used in 1973 and 1974. Both members of the survey party were familiar with them, having been students at the 1974 TTU Field School. Moreover, only one member of the field party kept the field notes and completed the recording forms; the other member was responsible for determining site dimensions and selecting material for collection.

The field supervisors, Paul and Susanna Katz, were responsible for all the logistical preparations, and they participated at the beginning and the end of the field survey. The members of the field party, Bryan Aivazian and Paul Lukowski, conducted the majority of the field survey by themselves, contacting the supervisors by telephone whenever they left the High Country. The inventory of the High Country was completed one day before another helicopter was scheduled to remove the equipment and collections. The helicopter removal phase was conducted without any mishap whatsoever, although the helicopter originally contracted for crashed and burned the day before it was scheduled to arrive. No one was injured in the crash; we are grateful to the National Park Service for finding a replacement helicopter at short notice so that our timetable was in no way upset.

\section{DESCRIPTION OF ARCHAEOLOGICAL DATA}

Sites

In our previous report (S. Katz and P. Katz 1974:12-13) a site was defined:

... by the presence of artifacts in quantity and concentration, whether or not features were associated. Features without associated artifacts were not elevated to site status.

During this past survey, and for the purposes of the present report, much greater emphasis was placed on the feature component in defining a site than was previously the case. Very few researchers would fail to assign site status to a midden ring, in that it is assumed to represent repeated and patterned 
subsistence activities over a period of time in a restricted spatial context. Strict adherence to the above definition would, however, disqualify as a site any midden ring which was not associated with artifactual material, as would have indeed been the case with site 41 CU 202. A distinction will be made below between a major feature (e.g.s midden ring or mound) and a minor feature (e.g., hearth), but this is a detail which should not complicate a working definition. Strict adherence to the above definition would likewise disqualify as a site a small rockshelter with a smoke-blackened ceiling, one pictograph on the wall and no artifacts. These examples suggest that what archaeologists call a "site" is less a matter of associated material and more that it represents a set of spatial, temporal and behavioral characteristics. The following definition applies to the current project, but in no way abrogates any previous data collected in a comparable manner:

A site is characterized by the physical remains of patterned human behavior occurring in a localized, finite area, and from these physical remains postulations can be made as to the time-span involved and activities performed.

At a level of classification below the "site" is the collecting locality, employed in 1976 as it had been defined for the previous survey ( $S$. Katz and $P$. Katz 1974:13):

Extensive light scatterings of artifacts without associated features...; these included linear collecting localities (e.g., fence lines, trails) and areal collecting localities (e.g., Tegal sections).

The lack of spatial definition, the ephemeral nature of the physical remains, and the usually indeterminate aspects of time and function all characterize a collecting locality; by definition they do not constitute a site. It should be emphasized that a collecting locality has no definable boundary from a cultural point of view; that is, one cannot say, "Here is where the artifactual material ceases and the locality ends." This category is a device for recording cultural items in space, where the space is artificially determined and no internal pattern of aboriginal behavior can be discerned. The amount of material present within a collecting locality will usually be directly proportional to the amount of territory involved; a corollary characteristic is the absence of any concentration of material within the locality.

The third, and lowest, level of spatial classification employed during the survey was that of isolated find. This designation was applied to the recovery location of a single artifact, or at most a small number of artifacts, simply to provide a means of recording the existence of the specimen(s). An isolated find is assumed to represent an unintentional loss on the part of an aboriginal, or at best a minimal tool maintenance operation while in transit.

During the following discussion of site characteristics, a distinction will be made between newly recorded sites and previously recorded sites. Sixty-seven sites were recorded for the first time in 1976, although two of them were outside that portion of the Park defined as the High Country (Fig. 2). These latter two--41 CU 12 and 41 CU 197-mare discussed in separate appendices, and their characteristics are not included among the data tabulated for the 65 
High Country sites. Twenty sites had been recorded above 6500 feet elevation prior to 1976, and selected data has been provided (Table 2) for use in the summary discussions to follow. Of these 20, nine were revisited during 1976 and additional data on artifacts recovered is presented in separate tabulations (Tables 13, 16, 18). For convenience only, the word "site" should be taken from this point forward to mean "newly recorded High Country sites"; modifiers will be employed when referring to previously recorded or other groups of sites.

The collecting localities and isolated finds will not be discussed to nearly the same degree as will the sites. Neither the specificity of locational data nor their interpretive potential is comparable. Figure 3 depicts their position within the survey area, and tabulations are provided of the associated artifactual material recovered from them (Tables 14,17 ).

\section{Physical Aspects}

Before describing and classifying the cultural aspects of the sites, some of their physical characteristics and variations should be presented. These include: topographic situation, topographic orientation, elevation, and the areal extent of observed activities and/or cultural material.

The locations of the 65 High Country sites could be grouped into five major topographic situations, three of them having subcategories. Figure 4 provides a visual depiction of their relationship to each other; Table 3 lists the sites associated with each situation.

Topographic orientation is a locational attribute which combines the linear axis of the topographic situation with the placement of the site in relation to that axis. For instance, if a ridge line is oriented north-south, the site may be situated on either the east or west flank; a stream channel running east-west may have a site located on either its north- or south-side terrace. Solution cavities may form in either wall of a canyon which runs north-south, but sites may indicate a preference for only east-facing rockshelters. Table 4 provides this data for certain topographic situations; some are difficult to determine for lack of a linear orientation, like saddle centers or summit crests.

The elevation above mean sea level of newly recorded High Country sites is provided in Table 5, grouped into ranges of 250-foot increments. The site areas (in square meters) are also presented as grouped data (Table 6), al though the large overal1 range necessitated the group ranges increasing logarithmically rather than arithmetically. In all but three cases, site area was determined by the extent of chipped stone debris. Two of the exceptions were rockshelters, one of which (41 CU 236) could not be measured at a11 due to the hazards involved. The third site was $41 \mathrm{CU} 202$, a midden ring with no observable artifactual material on the surface; its area reflects only the feature itself.

Summarizing the data presented in Tables 3-6, prior to a consideration of the types of sites involved, it can be seen that the saddle location is by far the most popular (over 43\%), followed by ridge and stream terrace situations, respectively. Little preference was shown for summits; vertical faces are actually rare in the High Country, relative to the bordering escarpments. Where orientations could be determined, there was an overwhelming preference 


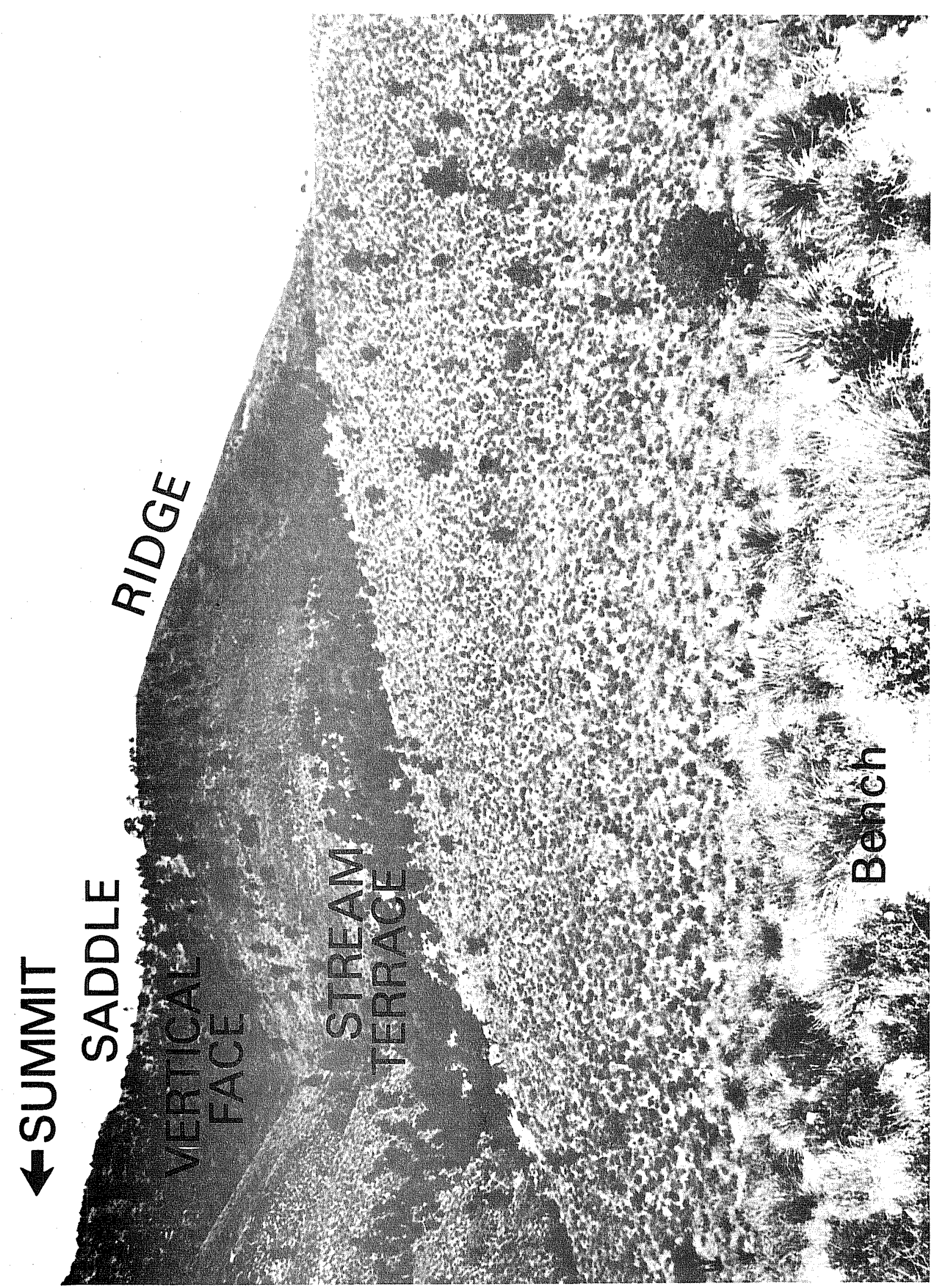


TABLE 3. CORRELATIUN OF NEWLY RECORDED HIGH COUNTRY SITES AND TOPOGRAPHIC SITUATIONS (SEE FIG.4)

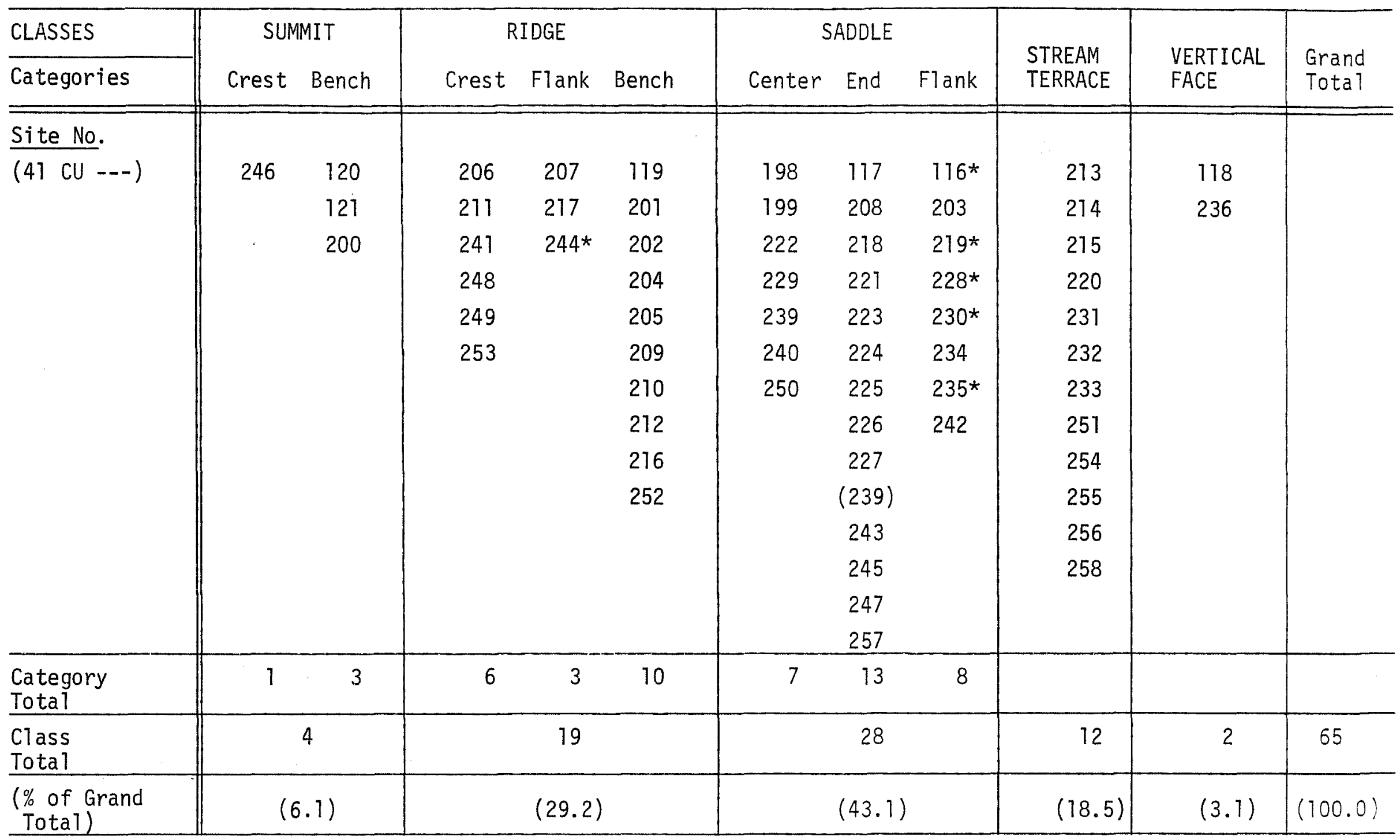

() = Site classified in another category

* = Associated with head of drainage 
TABLE 4. SITE POSITIONS WITH RESPECT TO TOPOGRAPHIC ORIENTATION, FOR SELECTED TOPOGRAPHIC SITUATIONS

\begin{tabular}{|c|c|c|c|c|c|}
\hline Site No. & $\begin{array}{l}\text { Bench, Ridge, } \\
\text { or Saddle } \\
\text { Orientation }\end{array}$ & $\begin{array}{l}\text { Site } \\
\text { Position }\end{array}$ & Site No. & $\begin{array}{l}\text { Stream } \\
\text { Orientation }\end{array}$ & $\begin{array}{l}\text { Site } \\
\text { Position }\end{array}$ \\
\hline
\end{tabular}

\section{Summit Bench}

$\begin{array}{lll}41 & \text { CU } 120 & \text { South-facing } \\ 41 \text { CU } 121 & \text { South-facing } \\ 41 \text { CU } 200 & \text { South-facing }\end{array}$

\section{Ridge Flank or Bench}

$\begin{array}{llcl}41 & \text { CU } 119 & \text { E-W } & \text { S } \\ 41 & \text { CU } 207 & \text { NE-SW } & \text { NW } \\ 41 & \text { CU } 217 & \text { E-W } & \text { N } \\ 41 & \text { CU } 244 & \text { E-W } & \text { N }\end{array}$

\section{Saddle Flank}

\begin{tabular}{|c|c|c|}
\hline 41 CU 116 & $E-W$ & N \\
\hline 41 CU 203 & $N E-S W$ & SE \\
\hline 41 CU 219 & $E-W$ & $E$ \\
\hline 41 CU 228 & NW-SE & NE \\
\hline 41 CU 230 & NW-SE & SW \\
\hline 41 CU 234 & $\mathrm{~N}-\mathrm{S}$ & $E$ \\
\hline 41 CU 235 & NW-SE & NE \\
\hline 41 CU 242 & $\mathrm{~N}-\mathrm{S}$ & $E$ \\
\hline
\end{tabular}

$E$

$\mathrm{NE}$

SW

NE

\section{Stream Terrace}

41 CU 213

$\mathrm{N}-\mathrm{S} \quad \mathrm{W}$

41 CU 214

41 CU 215

41 CU 220

41 CU 231

41 CU 232

41 CU 233

41 CU 251

41 CU 254

41 CU 255

41 CU 256

41 CU 258

$\mathrm{N}-\mathrm{S}$

$\mathrm{N}-\mathrm{S}$

N-S

E-W

$\mathrm{N}-\mathrm{S}$

E-W

N-S

NE-SW

$\mathrm{N}-\mathrm{S}$

E-W

N-S 
TABLE 5. CORRELATION OF NEWLY RECORDED HIGH COUNTRY SITES WITH GROUPED ELEVATION

\begin{tabular}{|c|c|c|c|c|c|c|c|c|}
\hline $\begin{array}{l}\text { Elevation } \\
\text { Groups } \\
\text { (feet above ms l) } \\
\end{array}$ & $\begin{array}{l}6750- \\
7000 \\
\end{array}$ & $\begin{array}{l}7000- \\
7250 \\
\end{array}$ & $\begin{array}{l}7250- \\
7500 \\
\end{array}$ & $\begin{array}{l}7500- \\
7750 \\
\end{array}$ & $\begin{array}{l}7750- \\
8000 \\
\end{array}$ & $\begin{array}{l}8000- \\
8250\end{array}$ & $\begin{array}{l}8250- \\
8500 \\
\end{array}$ & $\begin{array}{l}\text { Grand } \\
\text { Total } \\
\end{array}$ \\
\hline $\begin{array}{l}\text { Site No. } \\
(41 \mathrm{CU}--- \text { ) }\end{array}$ & $\begin{array}{l}235 \\
245 \\
251\end{array}$ & $\begin{array}{l}199 \\
200 \\
212 \\
213 \\
214 \\
215 \\
220 \\
231 \\
250 \\
252 \\
253 \\
254 \\
255\end{array}$ & $\begin{array}{l}201 \\
203 \\
219 \\
221 \\
223 \\
224 \\
226 \\
232 \\
233 \\
243 \\
244 \\
248 \\
256\end{array}$ & $\begin{array}{l}119 \\
202 \\
204 \\
205 \\
206 \\
217 \\
218 \\
222 \\
225 \\
240 \\
241 \\
242 \\
246 \\
247 \\
249 \\
258 \\
\end{array}$ & $\begin{array}{l}120 \\
121 \\
207 \\
211 \\
216 \\
234 \\
257\end{array}$ & $\begin{array}{l}116 \\
117 \\
118 \\
198 \\
208 \\
209 \\
210 \\
227 \\
236 \\
239\end{array}$ & $\begin{array}{l}228 \\
229 \\
230\end{array}$ & \\
\hline $\begin{array}{l}\text { Group } \\
\text { Total } \\
\end{array}$ & 3 & 13 & 13 & 16 & 7 & 10 & 3 & 65 \\
\hline $\begin{array}{l}\text { (\% of } \\
\text { Grand } \\
\text { Tota } 1 \text { ) }\end{array}$ & $(4.6)$ & $(20.0)$ & $(20.0)$ & $(24.6)$ & $(10.8)$ & $(15.4)$ & $(4.6)$ & $(100.0)$ \\
\hline
\end{tabular}


TABLE 6. CORRELATION OF NEWLY RECORDED HIGH COUNTRY SITES WITH GROUPED AREA

\begin{tabular}{|c|c|c|c|c|c|c|c|c|c|c|}
\hline $\begin{array}{l}\text { Area Groups } \\
\left(\mathrm{m}^{2}\right)\end{array}$ & $\begin{array}{l}\text { Indeter- } \\
\text { minate }\end{array}$ & $\begin{array}{l}0- \\
499 \\
\end{array}$ & $\begin{array}{l}500- \\
999 \\
\end{array}$ & $\begin{array}{l}1000- \\
1999 \\
\end{array}$ & $\begin{array}{l}2000- \\
2999 \\
\end{array}$ & $\begin{array}{l}3000- \\
4999 \\
\end{array}$ & $\begin{array}{l}5000- \\
9999 \\
\end{array}$ & $\begin{array}{l}10,000- \\
19,999 \\
\end{array}$ & $\begin{array}{l}\text { Exceeds } \\
20,000 \\
\end{array}$ & $\begin{array}{l}\text { Grand } \\
\text { Total }\end{array}$ \\
\hline $\begin{array}{l}\text { Site No. } \\
(41 \mathrm{CU}---)\end{array}$ & 236 & $\begin{array}{l}116 \\
118 \\
202 \\
203 \\
205 \\
209 \\
210 \\
214 \\
223 \\
224 \\
226 \\
227 \\
229 \\
233 \\
242 \\
\end{array}$ & $\begin{array}{l}120 \\
204 \\
206 \\
208 \\
216 \\
219 \\
230 \\
243 \\
245 \\
248 \\
250 \\
254 \\
255 \\
256\end{array}$ & $\begin{array}{l}117 \\
119 \\
121 \\
207 \\
220 \\
222 \\
232 \\
239 \\
240 \\
241 \\
244 \\
249\end{array}$ & $\begin{array}{l}225 \\
247 \\
251 \\
252 \\
253 \\
257 \\
258\end{array}$ & $\begin{array}{l}200 \\
201 \\
213 \\
217 \\
231 \\
234 \\
235\end{array}$ & $\begin{array}{l}198 \\
199 \\
211 \\
212 \\
221 \\
228\end{array}$ & $\begin{array}{l}215 \\
246\end{array}$ & 218 & \\
\hline $\begin{array}{l}\text { Group } \\
\text { Total }\end{array}$ & 1 & 15 & 14 & 12 & 7 & 7 & 6 & 2 & 1 & 65 \\
\hline $\begin{array}{l}\text { (\% of } \\
\text { Grand } \\
\text { Total) }\end{array}$ & (1.5) & $(23.1)$ & $(21.5)$ & (18.5) & $(10.8)$ & (10.8) & $(9.2)$ & $(3.1)$ & $(1.5)$ & $(100.0)$ \\
\hline
\end{tabular}


for locations which took advantage of the early morning (east-facing) or noonday (south-facing) sun. Almost two-thirds of the recorded sites fell within the range of elevation between 7000 and 7750 feet; the same percentage of sites (over 64\%) measured less than 1000 square meters in area. The resultant picture is one of numerous small sites, situated between the peaks and canyon bottoms and taking advantage of sheltered situations which provided the maximum amount of sunlight. The proximity of water did not seem to be a dominant factor in site selection.

\section{Material Aspects}

Turning now to the material characteristics of the sites, one factor appears as practically constant: all but one of the 65 sites had chipped stone debris in association. An initial classification of the sites can thus be made on the basis of whether or not features are present. The class of sites with associated features was subdivided according to the type and number of features recorded: multiple midden, single midden, hearth or knapping area. The other general class of sites, those without features present, includes both open and closed site situations. The former are-designated lithic scatters (Fig. 5,a) to provide continuity with the previous report; the latter are rockshelters (Fig. 5, b). Table 7 provides a listing of recorded sites, correlated with the above categories.

It should be noted that the lithic scatter type of site has some similarities with the collecting locality; both are characterized by predominantly chipped stone artifactual material, and both lack midden features. A collecting locality, however, lacks any kind of feature and has its boundaries defined by artificial parameters. A lithic scatter site, on the other hand, has a discernible termination of material, most likely has internal activity areas or concentrations of material, and can have one or more interior features (e.g., the hearth at 41 CU 226 or the knapping area at 41 CU 239).

Middens are considered major features; their size, complexity and postulated method of construction all argue for repetitive activity over a period of time. The general class of midden feature has been subdivided into three categories: midden rings (Fig. 6,a) have their maximum height above the present ground surface toward their periphery; midden mounds (Fig. 6,b) have their maximum height above the present ground surface in their center; and indeterminate middens are accumulations of burned rock which cannot be assigned to either of the above two categories, but which do have some height above the present ground surface. No burned rock scatters, as defined in the previous report (S. Katz and P. Katz 1974:22), were documented in the High Country. The distinction between an indeterminate midden and a burned rock scatter is one of concentration of the burned rock and a measurable height above the present ground surface. Table 7 makes no distinction between the types of midden, but rather addresses itself to whether only one, or more than one, midden feature was recorded at a particular site. Subsequent tabulations (Tables $8,9,10$ ) are concerned with middens and their variations.

It must be emphasized that, without some testing, surficial midden rings can rarely be subdivided into varieties which either have a subsurface central pit (Greer 1965:Type 1; Weir 1976a:Type 3) or do not (Weir 1976a:Type 2). 


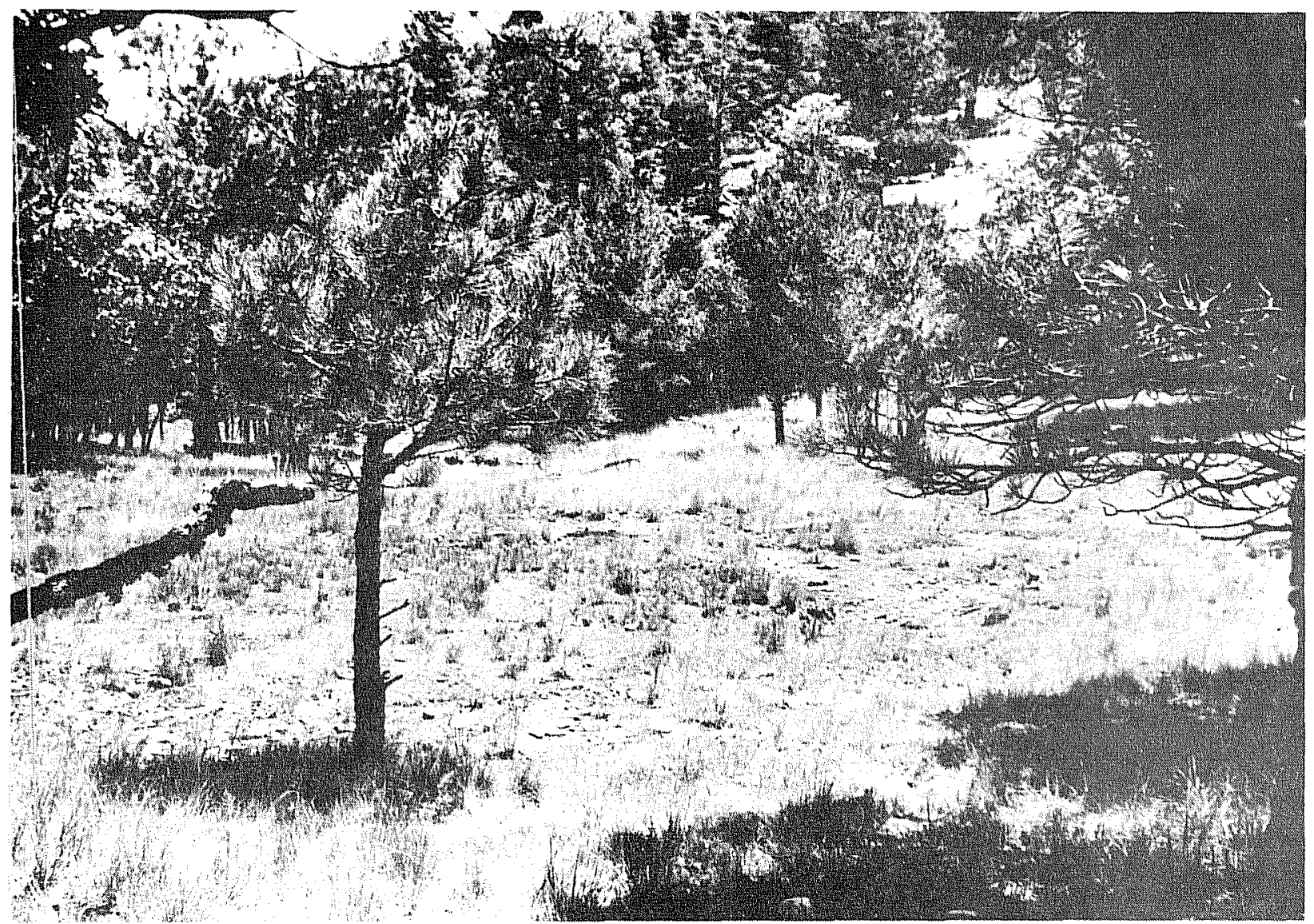

a

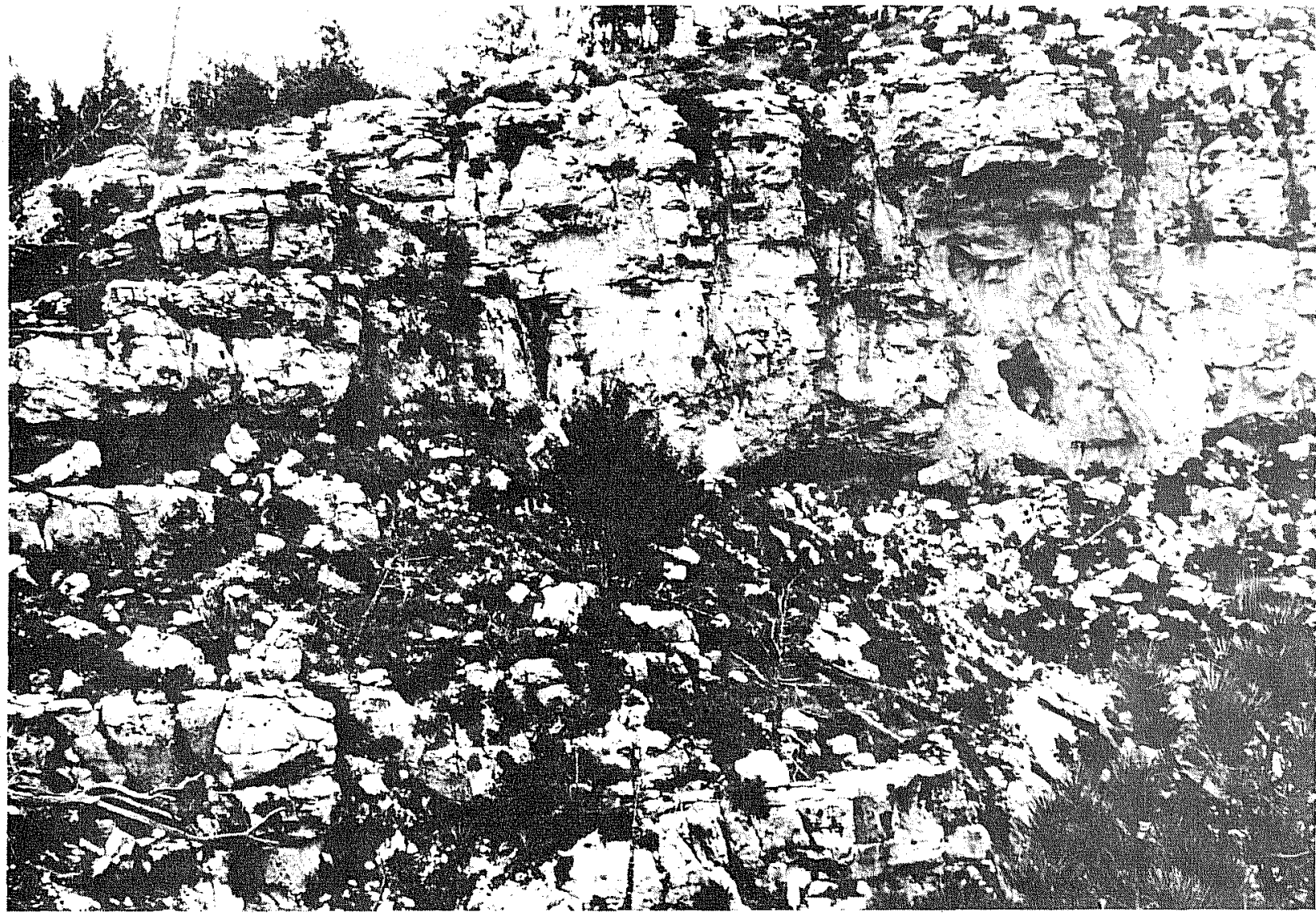

Figure 5. Non-feature Site Types. a, open lithic scatter (41 CU 235); b, rockshelter (41 CU 236). 
1.7.1. 13.

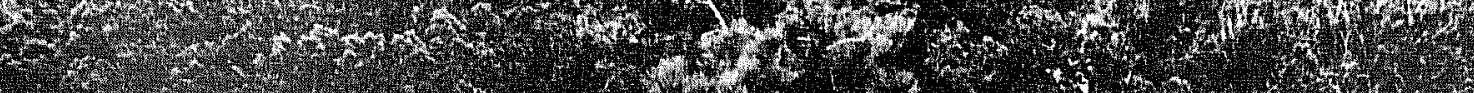

(18)

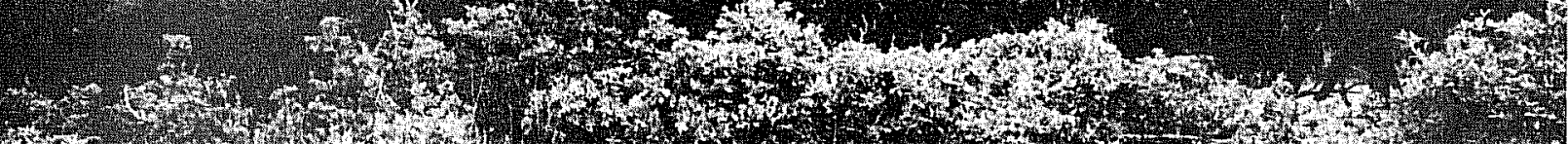
7.

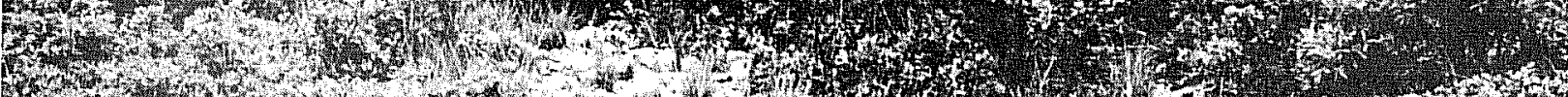
17. F. -

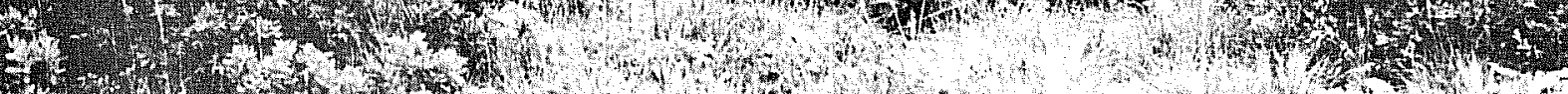
F.

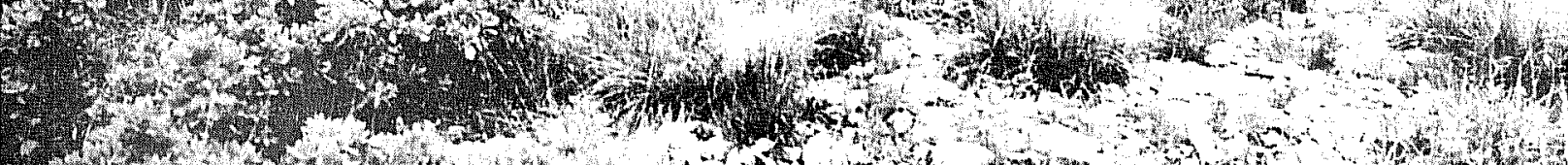

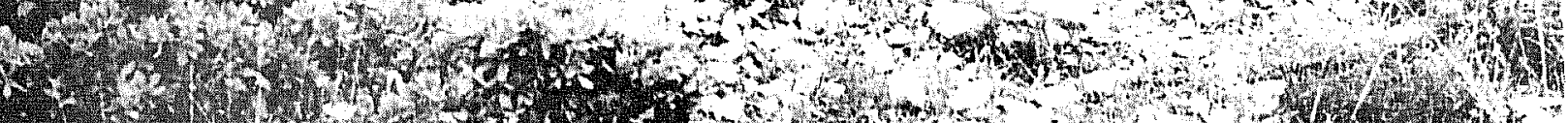
1.7.

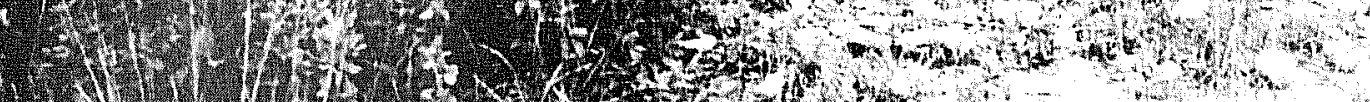

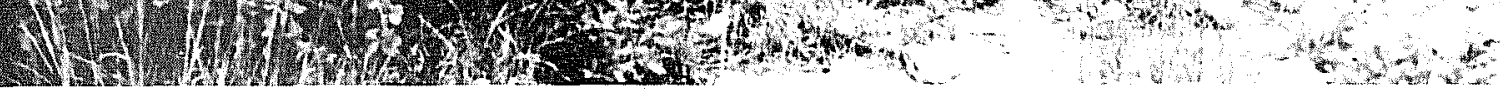

a

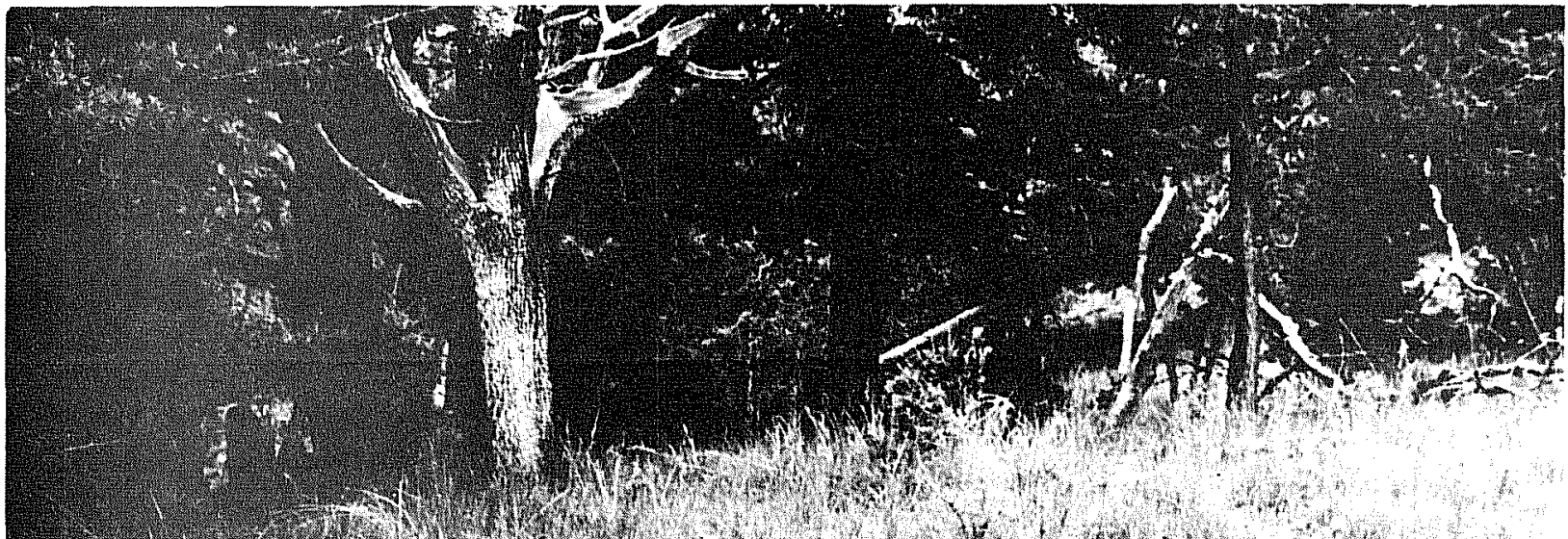

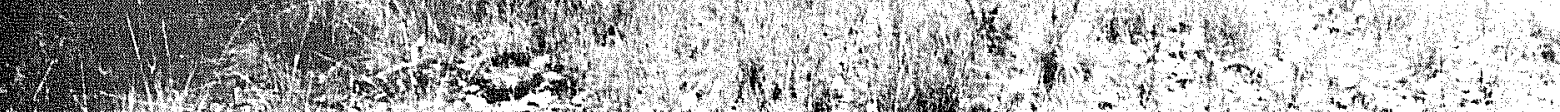

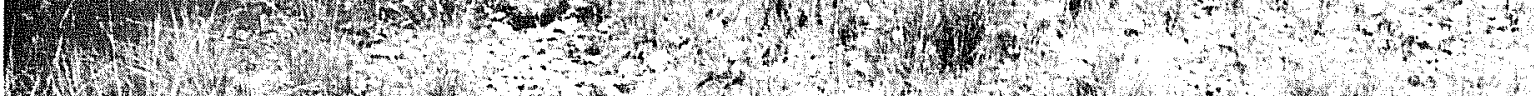

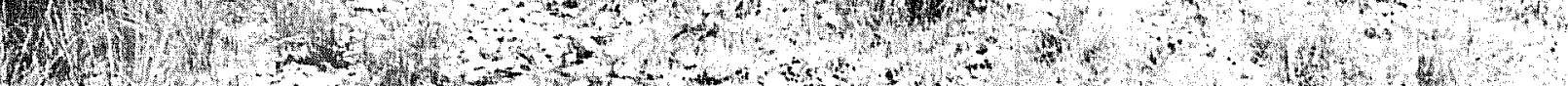
3
3

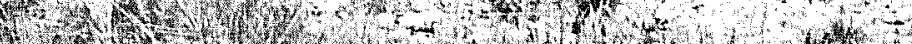

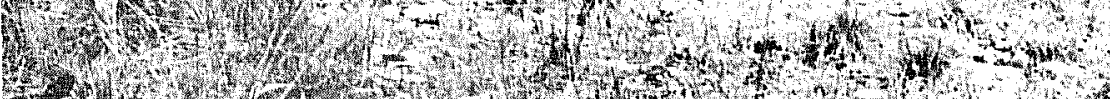

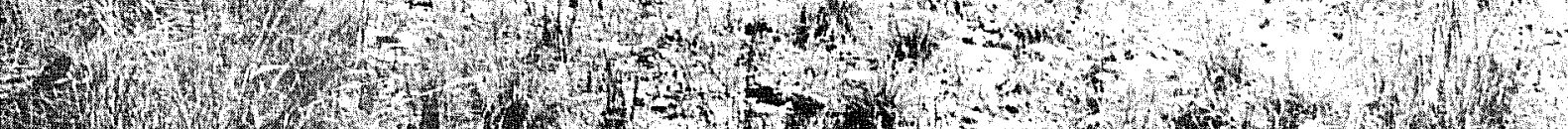

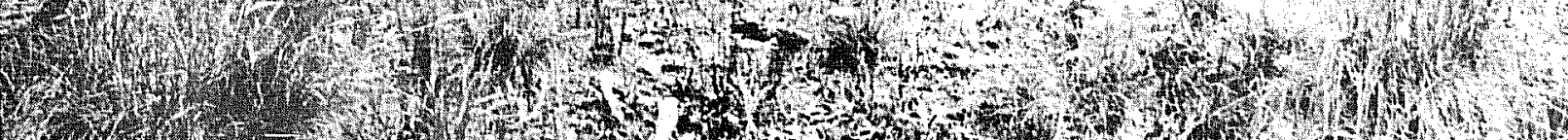

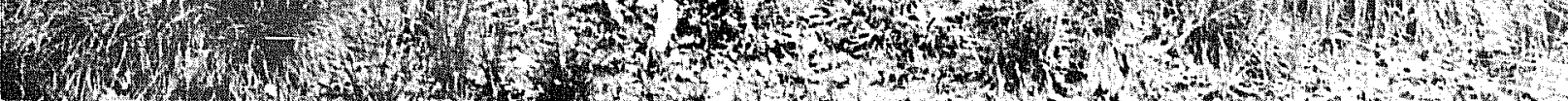

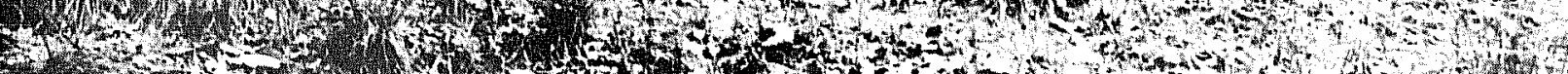


TABLE 7. CLASSIFICATION OF NEWLY RECORDED HIGH COUNTRY SITES ACCORDING TO FEATURE P/A AND TYPE

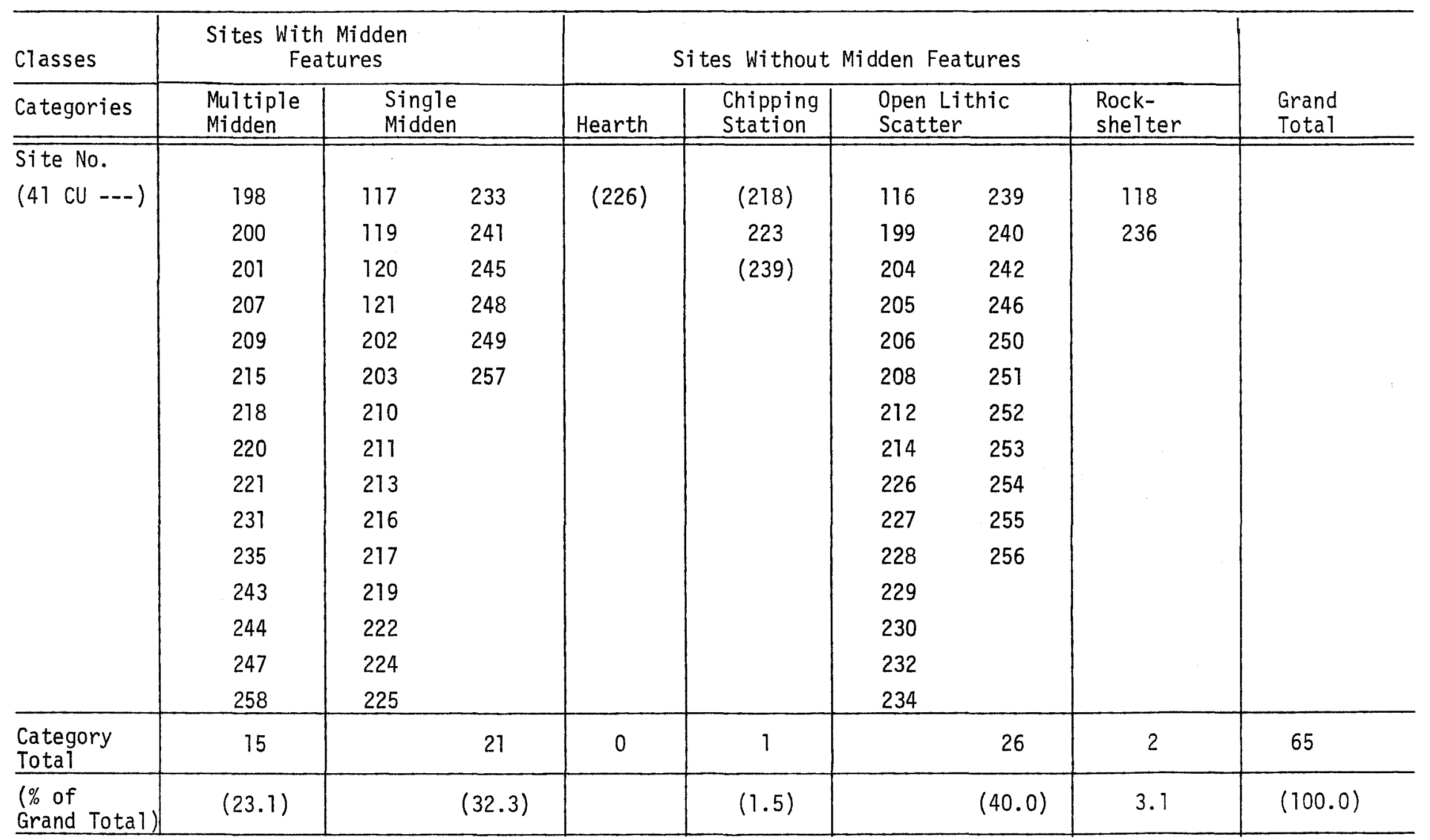

()$=$ Site classified in another category. 
TABLE 8. CLASSIFICATION OF NEWLY RECORDED HIGH COUNTRY MONOTYPIC MIDDEN SITES

\begin{tabular}{|c|c|c|c|c|c|c|c|c|}
\hline $\begin{array}{l}\text { Classes and } \\
\text { Categories }\end{array}$ & $\begin{array}{l}1 \\
\text { Ring }\end{array}$ & ${ }_{\text {Ring }}^{2}$ & $\begin{array}{l}3 \\
\text { Ring }\end{array}$ & $\begin{array}{c}4 \\
\text { Ring }\end{array}$ & $\begin{array}{l}1 \\
\text { Mound }\end{array}$ & $\begin{array}{l}1 \\
\text { Indeter- } \\
\text { minate }\end{array}$ & $\begin{array}{l}2 \\
\text { Indeter- } \\
\text { minate }\end{array}$ & $\begin{array}{l}\text { Grand } \\
\text { Total }\end{array}$ \\
\hline $\begin{array}{l}\text { Site No. } \\
\text { (41 CU ---) }\end{array}$ & $\begin{array}{l}119 \\
120 \\
121 \\
203 \\
211 \\
216 \\
217 \\
224 \\
225 \\
233 \\
245 \\
248 \\
249\end{array}$ & $\begin{array}{l}200 \\
209 * \\
235 \\
243^{*}\end{array}$ & $\begin{array}{l}201^{*} \\
207^{*} \\
220+ \\
244^{*}\end{array}$ & $247 \star \star$ & 210 & $\begin{array}{l}117 \\
202 \\
213 \\
219 \\
222 \\
241 \\
257\end{array}$ & $198^{*}$ & \\
\hline $\begin{array}{l}\text { Category } \\
\text { Total }\end{array}$ & 13 & 4 & 4 & 1 & 1 & 7 & 1 & \\
\hline $\begin{array}{l}\text { Class } \\
\text { Tota } 1 \\
\end{array}$ & \multicolumn{4}{|c|}{22} & 1 & \multicolumn{2}{|l|}{8} & 31 Sites \\
\hline $\begin{array}{l}\text { Feature } \\
\text { Total }\end{array}$ & \multicolumn{4}{|c|}{37} & 1 & \multicolumn{2}{|l|}{$y$} & 47 Features \\
\hline
\end{tabular}

2 intersecting features at site

3 intersecting features at site

4 intersecting features at site 
TABLE 9. CLASSIFICATION OF NEWLY RECORDED HIGH COUNTRY POLYTYPIC MIDDEN SITES

\begin{tabular}{|c|c|c|c|c|c|c|}
\hline & 1 Mound & 3 Mound & $\begin{array}{l}1 \text { Indeter- } \\
\text { minate }\end{array}$ & $\begin{array}{l}3 \text { Indeter- } \\
\text { minate }\end{array}$ & $\begin{array}{l}4 \text { Indeter } \\
\text { minate }\end{array}$ & $\begin{array}{l}\text { Total No. } \\
\text { of Rings }\end{array}$ \\
\hline 1 Ring & 358 & 231 & & & $215^{*}$ & 3 \\
\hline 2 Ring & & & & 221 & & 2 \\
\hline 3 Ring & & & & & & \\
\hline 4 Ring & & & $218 *$ & & & 4 \\
\hline \multirow{3}{*}{$\begin{array}{l}\text { Total No. } \\
\text { of Non- } \\
\text { Rings }\end{array}$} & & & & & & 9 Rings \\
\hline & 1 & 3 & 1 & 3 & 4 & \\
\hline & \multicolumn{2}{|c|}{4 Mounds } & \multicolumn{2}{|c|}{8 Indeterminates } & & $\begin{array}{l}21 \text { Features } \\
5 \text { Sites }\end{array}$ \\
\hline
\end{tabular}

* 2 intersecting features 
TABLE 10. NEWLY RECORDED HIGH COUNTRY MIDDEN FEATURE DIMENSIONAL DATA

\begin{tabular}{|c|c|c|c|c|c|c|c|c|c|c|c|c|}
\hline & \multicolumn{4}{|c|}{ R I N G } & \multicolumn{4}{|c|}{$M O \cup N D$} & \multicolumn{4}{|c|}{ I N D.ETERM INATE } \\
\hline Site No. & $\begin{array}{l}\text { Number } \\
\text { of } \\
\text { Features }\end{array}$ & $\begin{array}{l}\text { External } \\
\text { Diameter } \\
(\mathrm{m})\end{array}$ & $\begin{array}{l}\text { Internal } \\
\text { Diameter } \\
(\mathrm{m})\end{array}$ & $\begin{array}{l}\text { Height } \\
\text { Above } \\
\text { Ground (m) }\end{array}$ & Site No. & $\begin{array}{l}\text { Number } \\
\text { of } \\
\text { Features } \\
\end{array}$ & $\begin{array}{c}\text { External } \\
\text { Diameter } \\
(\mathrm{m})\end{array}$ & $\begin{array}{l}\text { Height } \\
\text { Above } \\
\text { Ground (m) } \\
\end{array}$ & Site No. & $\begin{array}{l}\text { Number } \\
\text { of } \\
\text { Features } \\
\end{array}$ & $\begin{array}{l}\text { External } \\
\text { Diameter } \\
(\mathrm{m})\end{array}$ & $\begin{array}{l}\text { Height } \\
\text { Above } \\
\text { Ground (m }\end{array}$ \\
\hline $41 \mathrm{CU} 119$ & 1 & 6.5 & -- & 0.5 & $41 \mathrm{CU} 210$ & 1 & 11.5 & 1.0 & $41 \mathrm{CU} 117$ & 1 & -- & 0.3 \\
\hline 41 CU 120 & 1 & 7.0 & -- & 0.5 & 41 CU 231* & 3 & 6.0 & -- & $41 \quad C U 198$ & 2 & 9.0 & 0.3 \\
\hline $41 \mathrm{CU} 121$ & 1 & 7.5 & -- & 1.0 & & & 6.0 & -- & & & 8.0 & 0.3 \\
\hline \multirow[t]{2}{*}{41 CU 200} & 2 & 9.5 & -- & 0.7 & & & 3.0 & -- & 41 CU 202 & 1 & 10.5 & 0.7 \\
\hline & & 10.5 & -- & 0.7 & 41 CU 258* & 1 & 12.0 & 0.8 & $41 \mathrm{CU} 213$ & 1 & 11.0 & 0.5 \\
\hline \multirow[t]{3}{*}{$41 \mathrm{CU} 201$} & 3 & 8.0 & -- & -- & ----- & - & -- & & 41 CU 215* & 4 & 6.5 & -- \\
\hline & & 12.0 & -- & -- & $n=3$ & 5 & 5 & 2 & & & 7.5 & -- \\
\hline & & 15.0 & 6.0 & 1.5 & $\bar{x}=$ & & 7.7 & 0.9 & & & 9.0 & -- \\
\hline 41 CU 203 & 1 & 12.0 & 5.0 & 1.5 & $s=$ & & 3.5 & N/A & & & 22.0 & -- \\
\hline \multirow[t]{3}{*}{41 CU 207} & 3 & 7.0 & -- & 0.3 & & & & & $41 \mathrm{CU} 218^{*}$ & 1 & 7.5 & 0.3 \\
\hline & & 8.0 & -- & 0.3 & & & & & 41 CU 219 & 1 & 7.0 & 0.7 \\
\hline & & 12.0 & 5.5 & 0.7 & & & & & $41 \mathrm{CU} 221^{*}$ & 3 & 11.0 & 0.7 \\
\hline \multirow[t]{2}{*}{41 CU 209} & 2 & 8.5 & -- & 0.3 & & & & & & & 12.0 & 0.8 \\
\hline & & 9.5 & -- & 0.7 & & & & & & & 12.0 & 1.0 \\
\hline 41 CU 211 & 1 & 15.5 & -- & 1.0 & & & & & 41 CU 222 & 1 & 3.0 & 0.2 \\
\hline 41 CU $215^{\star}$ & 1 & 13.0 & 6.0 & 0.7 & & & & & $41 \mathrm{CU} 241$ & 1 & 6.0 & 0.2 \\
\hline 41 CU 216 & 1. & 6.5 & -- & 0.2 & & & & & $41 \mathrm{CU} 257$ & 1 & 7.0 & 0.5 \\
\hline \multicolumn{5}{|c|}{$\begin{array}{l}--=\text { Data not recorded } \\
\star=\text { Polytypic midden site }\end{array}$} & & & & & $\begin{array}{l}n=11 \\
\bar{x}= \\
x=\end{array}$ & 17 & $\begin{array}{l}16 \\
9.3 \\
4.0\end{array}$ & $\begin{array}{l}13 \\
0.5 \\
0.25\end{array}$ \\
\hline
\end{tabular}


TABLE 10. (continued)

\begin{tabular}{|c|c|c|c|c|c|c|c|c|c|c|}
\hline \multicolumn{5}{|c|}{$R I N G$} & \multicolumn{5}{|c|}{$R I N G$} & \\
\hline Site No. & $\begin{array}{l}\text { Number } \\
\text { of } \\
\text { Features }\end{array}$ & $\begin{array}{l}\text { External } \\
\text { Diameter } \\
(\mathrm{m})\end{array}$ & $\begin{array}{l}\text { Internal } \\
\text { Diameter } \\
(\mathrm{m})\end{array}$ & $\begin{array}{l}\text { Height } \\
\text { Above } \\
\text { Ground (m) }\end{array}$ & Site No. & $\begin{array}{l}\text { Number } \\
\text { of } \\
\text { Features }\end{array}$ & \multirow{2}{*}{$\begin{array}{c}\begin{array}{c}\text { External } \\
\text { Diameter } \\
(\mathrm{m})\end{array} \\
11.5\end{array}$} & \multirow{2}{*}{$\begin{array}{c}\text { Internal } \\
\text { Diameter } \\
(\mathrm{m})\end{array}$} & $\begin{array}{l}\text { Height } \\
\text { Above } \\
\text { Ground (m) }\end{array}$ & \\
\hline 41 CU 217 & 1 & 8.0 & -- & 0.3 & 41 CU 243 & 2 & & & 1.0 & \\
\hline \multirow{4}{*}{$41 \mathrm{CU} 218^{*}$} & 4 & 9.0 & -- & 0.3 & & & 8.0 & 5.0 & 1.0 & \\
\hline & & 11.0 & -- & 0.7 & 41 CU 244 & 3 & 11.0 & 5.5 & 1.0 & \\
\hline & & 11.0 & -- & 0.7 & & & 10.5 & 5.5 & 1.0 & \\
\hline & & 11.5 & -- & 0.7 & & & 7.5 & -- & 0.3 & \\
\hline \multirow[t]{3}{*}{$41 \mathrm{CU} 220$} & 3 & 6.0 & -- & -- & 41 CU 245 & 1 & 11.0 & 5.5 & 0.8 & \\
\hline & & 11.0 & -- & -- & $41 \mathrm{CU} 247$ & 4 & 15.5 & 5.5 & 3.0 & \\
\hline & & 12.5 & 5.0 & 1.2 & & & 13.5 & 6.5 & 2.2 & \\
\hline \multirow{2}{*}{41 CU $221^{*}$} & 2 & 6.0 & -- & 0.3 & & & 11.5 & 6.0 & 0.7 & \\
\hline & & 13.0 & 6.0 & 1.0 & & & 11.0 & 5.5 & 2.0 & \\
\hline 41 CU 224 & 1 & 13.0 & -- & 0.3 & 41 CU 248 & 1 & 8.0 & -- & 0.2 & \\
\hline $41 \mathrm{CU} 225$ & 1 & 8.0 & 3.5 & 0.5 & 41 CU 249 & 1 & 12.0 & 5.5 & 1.0 & \\
\hline $41 \mathrm{CU} 237^{*}$ & 1 & 10.5 & -- & 0.7 & $41 \mathrm{CU} 258^{*}$ & 1 & 13.0 & 5.5 & 1.2 & \\
\hline 41 CU 233 & 1 & 9.0 & 4.0 & 1.0 & ----- & ----- & $-\cdots--$ & ---- & ---- & \\
\hline \multirow[t]{5}{*}{41 CU 235} & 2 & 11.0 & -- & 1.0 & $n=$ & 46 & 46 & 20 & 42 & \\
\hline & & 9.5 & 4.0 & 1.2 & $\bar{x}=$ & & 10.3 & 5.3 & 0.9 & \\
\hline & & & & & $s=$ & & 2.5 & 0.7 & 0.6 & \\
\hline & $S \cup M$ & $A R Y$ & & & & & & & & \\
\hline & Ring & Mound & Indeterm. & Tota & & & & & & \\
\hline No. Sites & 27 & 3 & 11 & 36 & & & & & & \\
\hline No. Features & 46 & 5 & 17 & 00 & & & & & & \\
\hline
\end{tabular}


While some midden rings are characterized by central depressions which extend all the way to ground surface, this in itself is no guarantee of subsurface excavation and activity. To complete the comparison between the above classification and another one employed in central Texas, the midden mound corresponds to Weir's (1976a) Type 1 midden, while the burned rock scatter is Weir's (1976a) Type 4 midden.

The four minor features recorded in the High Country in 1976 include one hearth and three knapping areas (areas definable as being used exclusively for flintknapping activities). The designation "minor" is applied to indicate a smaller area, less internal complexity and most likely a shorter use time relative to midden features. Minor features were not normally given site status solely on the basis of their presence, although Table 7 appears to rank them equally with midden and non-midden sites. Thus, the hearth at $41 \mathrm{CU} 226$ is one component of a lithic scatter site; the identifiable chipping stations at 41 CU 218 and 41 CU 239 are part of a multiple midden site and a lithic scatter site, respectively. The sole exception is $41 \mathrm{CU} 223$, a site defined onty on the basis of a knapping area. Hindsight would suggest designating this activity area an isolated feature, a category fuily in keeping with the spatial classificatory scheme and one which had been employed successfully in 1973.

It is clear from Table 7 that the High Country sites recorded in 1976 are approximately equally divided between those with major burned rock features and those without. Considering the total number of recorded High Country sites (85), those with middens account for 59\% (50 sites), with 1 ithic scatters representing $34 \%$ (29 sites). Five shelters and caves account for $6 \%$; the remaining $1 \%$ is the chipping station (41 CU 223). We were frankly surprised to find that three out of every five High Country sites were characterized by at least one midden, a feature which is usually "located below 4,000 feet elevation within the optimum growing area for both sotol and mesca 1" (Applegarth 1976:161). For this reason, as we 71 as the fact that sites with middens are the most numerous, a more detailed discussion concerning them follows.

The single midden sites (Table 7) are, by definition, monotypic; only one type of midden feature occurs. Of the 21 single midden sites recorded, 13 are characterized by a midden ring, 7 by indeterminate middens and only one by a midden round (Table 8). A multiple midden site has the potential, however, of being polytypic, whereby more than one type of midden feature would be present. of the 15 multiple midden sites (Table 7), however, only five exhibit this characteristic. Of the 10 monotypic, multiple midden sites (Table 8), nine are midden rings (three doubles, three triples and a quadruple), and one has two indeterminate middens. At least one ring midden occurs at each of the five polytypic multiple midden sites (Table 9), in combination with one or more midden mounds or indeterminate middens; two of these five sites have more than one midden ring.

Another aspect of multiple midden sites is the possibility of feature intersection, and the obvious inferences about time depth that this occurrence provides. Nine of the 15 multiple midden sites exhibit evidence of feature intersection, with eight of these occurrences associated with midden rings. The intersection phenomenon seems to affect those sites with the largest number of features: all six sites with three or four midden rings; the one site with four indeterminate middens; and two of the five sites with two ring middens (Tables 
8,9). An immediate and obvious interpretation of the fact that sites with more features tend to have more intersections might be that the site location was worth repeated visits, but repeated activities could not be contained within the available space. Comparing Tables 8 and 9 (listing sites with intersecting features) with Table 6 (site area) does not support this interpretation: sites with two features have small areas; sites with three features fall in the area mid-range; and the two sites with five features each and each with an intersecting pair of rings (41 CU 215 and $41 \mathrm{CU} 218$ ) are two of the three largest sites in areal extent.

Pursuing this same line of investigation, that High Country topography might result in limitations being placed on site size and type of feature, the dimensions of all midden features were tabulated (Table 10). One might postulate that the overall size of these features would be reduced, so as to "fit" into locations where flat, open working space was at a premium. While there is no data available in the literature for midden mounds or indeterminate middens as defined in this study, the midden ring data does not seem at all diminutive (cf. Mera 1938; Greer 1965; Applegarth 1976).

Using the dimensional data in Table 10, one might speculate as to the original configuration of the presentiy indeterminate middens. Although the midden mound sample is rather small for generalizations, the available figures suggest that mound and ring heights are similar $(\bar{x}=0.9 \mathrm{~m})$, while the mound diameters average about $21 / 2$ meters smaller $\left(\bar{x}_{r}=10.3 \mathrm{~m} ; \bar{x}_{\mathrm{m}}=7.7 \mathrm{~m}\right)$. The indeterminate middens have a smaller mean height than both $(0.5 \mathrm{~m})$, but a mean external diameter closer to the rings $(9.3 \mathrm{~m})$. An eroding mound would be assumed to increase in external diameter while decreasing in height; an eroding ring might be expected to contract inward while decreasing in height, as its central depression filled with its peripheral material. Both conditions would unfortunately reflect the mean dimensional data available for indeterminate middens. On the other hand, the fact that midden mounds and rings have the same mean height suggests that they are indeed distinct features; mounds should not be considered as eroded rings.

The data from the 14 previously recorded High Country midden sites (Table 2) follows closely the patterns seen above in the 1976 data. Multiple midden sites (five) tend to have larger site areas, and four of these five are characterized by intersecting features. Of the nine monotypic midden sites, six are rings, two are indeterminate middens, and one is a mound. There is at least one ring at each of the five polytypic midden sites. It should be mentioned that Mera (1938) excavated a single midden ring inside High Cave, situated on the western escarpment. While middens in front of shelter or cave mouths do occur in low frequencies in the region (e.g., Applegarth 1976:Table 32 records a 32\% occurrence), the High Cave midden is highly unusual. In that the remaining $99 \%$ of the High Country middens occur at open sites, the High Cave feature will not be included in the summary discussions below.

Table 11 presents counts and percentages for midden sites and their associated features. Ninety-five midden features have been recorded in the High Country, at 50 open sites. While these figures indicate an average of two midden features at every midden site, in point of fact there are $20 \%$ more single midden sites than multiple sites. All the single feature sites have only one type of midden by definition, but half of the multiple midden sites also fall into the mono- 
TABLE 11. SUMMARY OF MIDDEN FEATURE POPULARITY AT HIGH COUNTRY SITES

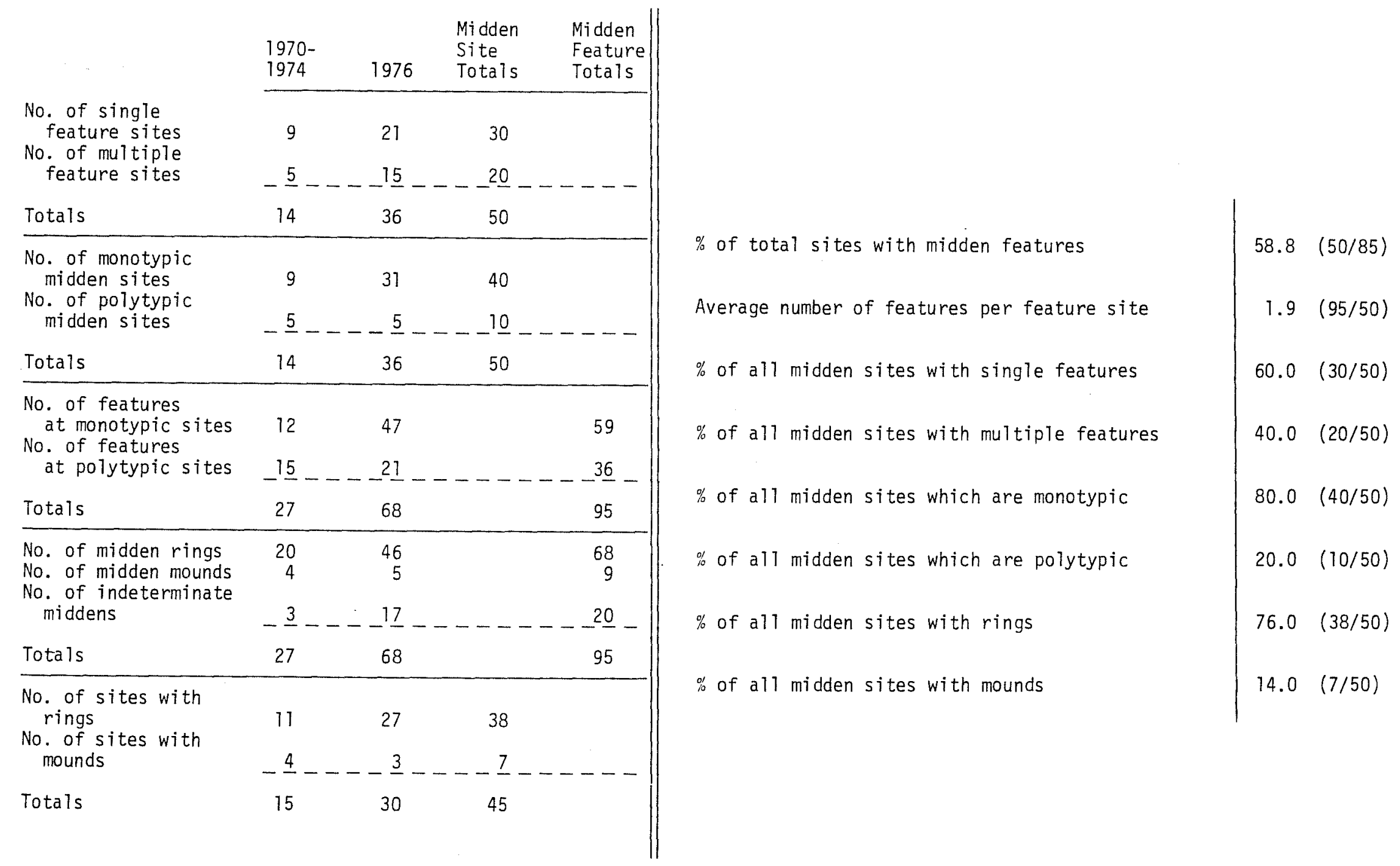


typic category. A full $80 \%$ of the midden sites have only one kind of midden feature present, and it is the midden ring which is far and away the most common variety. Rings are present at a 1110 of the polytypic midden sites and at 28 of the 40 monotypic sites, for a total representation of $76 \%$ at High Country midden sites. The other distinct midden feature, the mound, is documented at only seven (14\%) of the midden sites. The figures for et ther type might be higher if some determination could be made for the 20 indeterminate middens recorded, but we are unwilling to do this without further study and, ideally, some selective test excavations.

Correlation of site types with topographic variables (situation, orientation, elevation) does not modify the summary description of High Country sites presented above. This summary was based on a majority of sites, and this majority is represented by those with midden features in general and rings in particular. Sites with no midden features present (overwhelmingly represented by open lithic scatters) occupy the same range of situations, orientations and elevations as do sites with midden features, just in slightly smaller numbers. While there does seem to be a rough correspondence between sites with larger areal extents and those with multiple middens, large size per se does not necessarily imply the presence of midden features; four of the nine largest sites (Table 6) are lithic scatters.

One final aspect of the High Country sites which has yet to be addressed is their location with respect to the overall geography of the area. Figure 7 depicts the locations of all 85 recorded sites, as well as the major physiographic features of the High Country. Two patterns of site distribution can be ascertained, one linear and the other conglomerate. The linear pattern correlates sites with the major ridge systems: Blue Ridge (the western escarpment), Guadalupe Ridge, McKittrick Ridge and the eastern escarpment. Ridges provide the most logical and convenient (and in some places, the only) circulation routes throughout the High Country; and aboriginal hunters and gatherers could be expected to exploit the most accessible resources to the greatest degree. The aboriginal circulation pattern is probably mirrored quite closely by the current Park trail system (Fig. 7). While this fact was beneficial during the archaeological reconnaissance, it presents a serious management problem which will be discussed in a subsequent section of this report.

What is referred to as a conglomerate pattern of site location has two aspects. Small clusters of sites seem to be located in the immediate vicinity of present trail junctions and designated camping areas (Fig. 7). Employing the analogy above, that current circulation and usage reflects that of the aboriginal visitors, it might be postulated that these served the same functions--logical stopping places. Trail junctions, in that they reflect ridge intersections, are natural landmarks; both the junctions and camping areas are more often broader and flatter than any other place in the High Country with a comparably small areal extent.

There are three large clusters of sites: in the Bowl; at the head of South McKittrick Canyon; and at the head of Devils Den Canyon. Not only are these three areas characterized by a relatively large absolute number of sites, but only one of the 15 sites with three or more midden features is not situated within one of the three clusters. Six are included in the South Mckittrick cluster (41 CU 215, 218, 220,221, 231, 258), five with the Devils Den cluster 


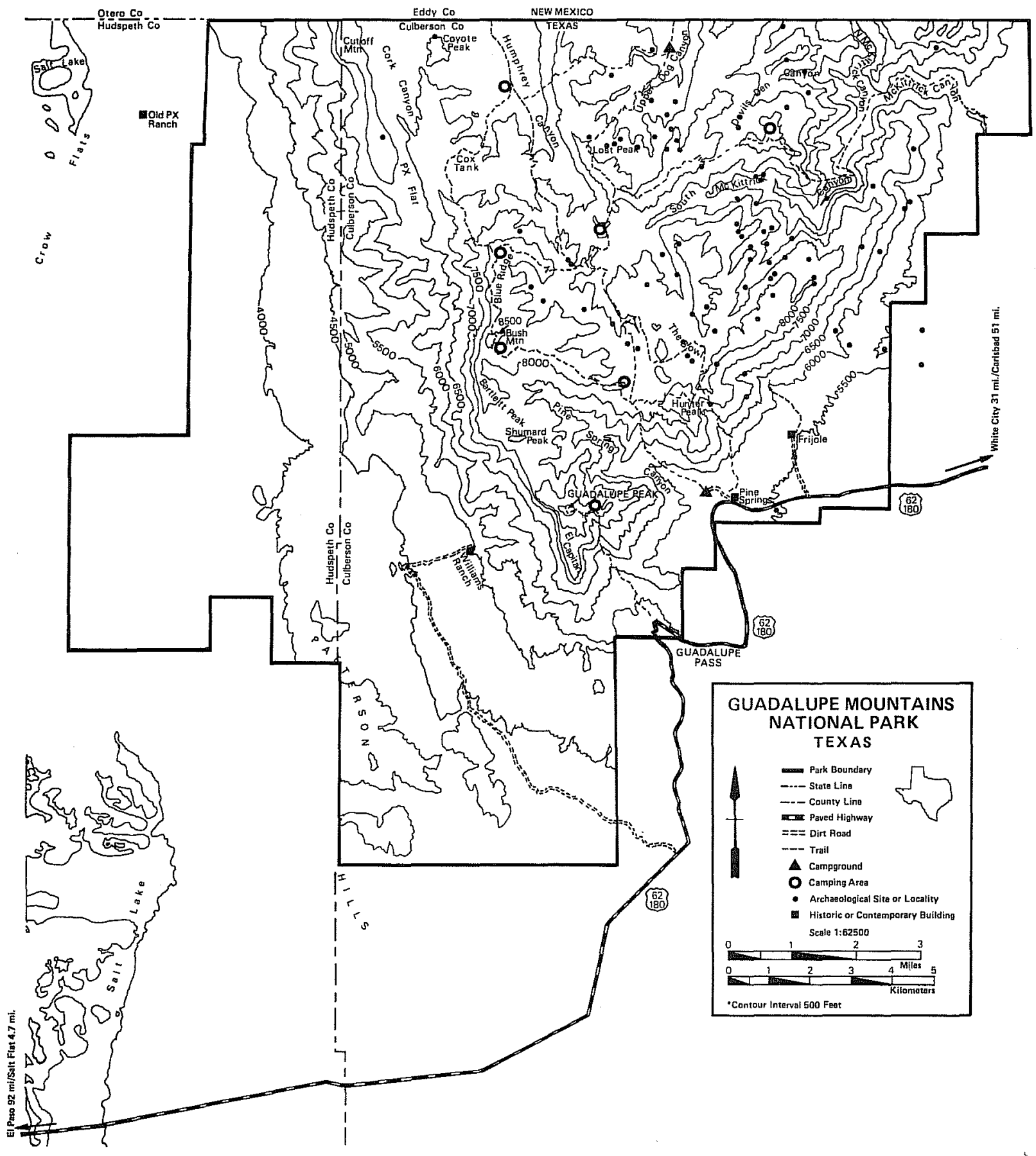

Figure 7. Locations of Recorded Archaeological Sites Over 6500 Feet Elevation. All projects are included. 
( 41 CU 140, 175, 201, 244, 247), and three in the Bow1 cluster (41 CU 80, 185, 207); 41 CU 169, with four features, is located southeast of Cox Tank on the ridge between Cork and Humphrey Canyons. It is not certain exactly why these major clusters occur. In the case of the two canyons, a number of factors are most likely responsible: access routes into the High Country; easily located landmarks; and the probability of a water supply at least visible from the site, if not in fairly close proximity. The Bowl might not reflect the access route factor, but it is undoubtedly sheltered, well watered with respect to other areas in the High Country, and one of the best known landmarks in the Park.

\section{Artifacts}

\section{Collection Procedures}

Artifactual material was collected at 63 of the 65 newly recorded High Country sites. The two exceptions were $47 \mathrm{CU} 202$ and 248, both single midden ring sites in the Devils Den cluster (Fig. 2). No artifacts were observed at the former site; so few were present at the latter that collection was deemed inadvisable.

The survey and collection procedures employed at each site followed the methodology of the 1973 inventory. As in 1973, onty two archaeologists were involved in recording and collecting each site; a similar methodology would be expected to produce more comparable data than would procedural diversity. The 1973 experiences and data recovery were deemed satisfactory enough to justify a continuance of that particular collection methodology.

The procedures consisted of first locating a possible site by the presence of a feature and/or a scatter of artifactual material. Both surveyors would then make a determination of the site's spatial extent by following the artifact scatter until it terminated. A provisional "site center" was established, on the basis of geometry or maximum artifact concentration or the location. of the feature(s); usually more than one of these variables were coincident. One person would then remain at the site center to record information, while the other collected the site area in a spiral pattern away from the center toward the periphery. As a final procedure, both persons would crisscross the site, taking photographs and making a final collection.

It should be noted that the personnel were aware of the variety of more rigorous, or at least more statistically valid, methods of site collection, such as the "dog leash," transects and gridding. For the reasons discussed above, as we 11 as the various adverse factors of limited time, dense ground cover and the sloping nature of most sites which would have presumably accounted for a high proportion of displaced artifacts, none of the more controlled collection methods were employed.

None of the sites recorded can be said to have been $100 \%$ collected, al though all were intensively surveyed. The primary physical characteristic of the sites which precluded complete collection was the dense ground cover, making the location of every flake an impossibility even when the survey was conducted on hands and knees. It was never the intention, however, to recover every cultural 
item present at a site; rather, the collection was oriented toward providing a range of raw materials, sizes, shapes and breakage patterns. Nevertheless, a11 chipped stone specimens which exhibited retouching, as wel1 as a representative sample of pottery types, were collected whenever located.

The assemblages were returned to the laboratory in San Antonio for analysis, where they were used to make determinations of a site's activity profile and occupational history. They wi17 also provide a comparative collection for the reanalys is of previously recorded sites, now in progress as part of the integrated report on the prehistory of the Park.

\section{Descriptive Categories}

For the purposes of classification and analysis control, each site collection was initially subdivided into three classes: chipped stone, rough stone and pottery. Each of these classes was then further subdivided into categories designed by a technological, morphological or functional descriptor. The lack of classificatory consistency at the category level results more from a desire to communicate effectively by using commonly employed terms than from any personal preference for inexactness on the part of the analyzers.

There are six categories of chipped stone artifacts. The most numerous specimens are unretouched flakes, which include both completely unmodified chipped stone debris and those flakes exhibiting lateral edge modification but no retouching. Exhausted cores (Fig. 8,b) fall at the other end of the technological spectrum from flake debris, in that they are the last item to be discarded during the process of reducing a nodule to produce workable flakes. The very low frequency of these items suggests that little core reduction was done at High Country sites; it is postulated that the chert sources were not readily available, and flake blanks rather than nodules were carried into the High Country.

The category of flakes which have been intentionally altered along one or more edges or ends are designated marginally retouched flakes. Identifiable subcategories include those specimens which exhibit flake scars on only one face (Fig. 9,a), as opposed to those with marginal bifacial retouching (Fig. 9,c). While this distinction is basically a morphological one, most researchers would agree that functional implications are likely. Unifacial marginal retouching produces a steeply angled working edge more suitable for scraping than the more acute, knife-like edge produced by marginal bifacial retouching.

A special case among the marginally retouched artifacts is the use of tabular or slab-Tike sections of limestone rather than chert or related silicious minerals. The blanks are thin, and the retouching is bifacial along one lateral margin (Fig. 8,a). This type of implement is common in low frequencies throughout the region and has often been referred to as a "limestone chopper" (Taylor 1966:69, Fig. 7).

Flakes exhibiting primary overall unifacial retouching fall into the fifth chipped stone category. Subcategories include blanks (Fig. 10) and completed tools with steep, secondary marginal retouching (Fig. 9,b). The latter are generally presumed to have been used to perform scraping functions, and the marginal retouching occurs in various combinations of end and side locations. 

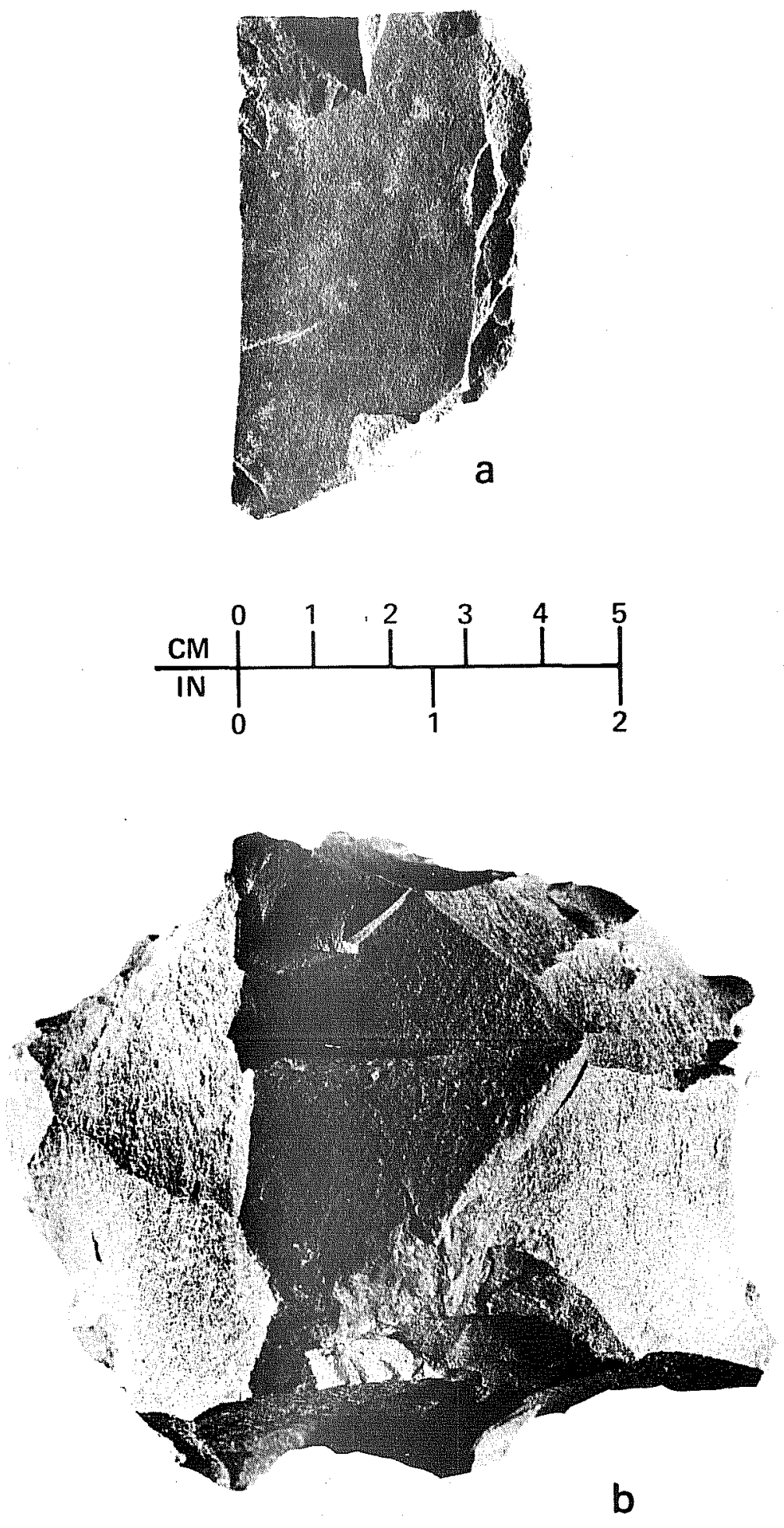

Figure 8. Chipped Stone Artifacts. a, limestone slab with marginal bifacial retouching; $b$, exhausted core. 


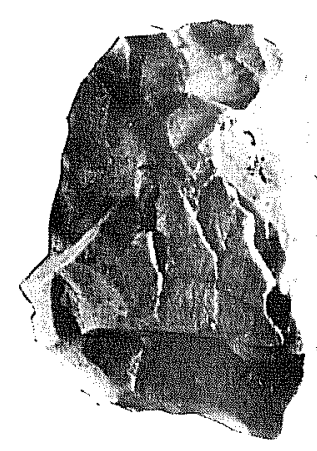

a

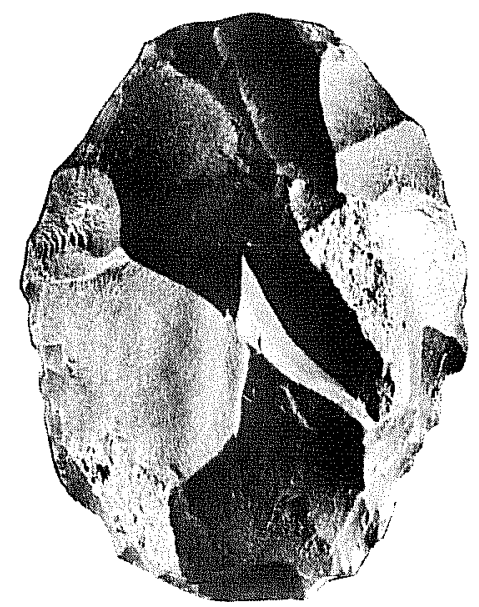

b
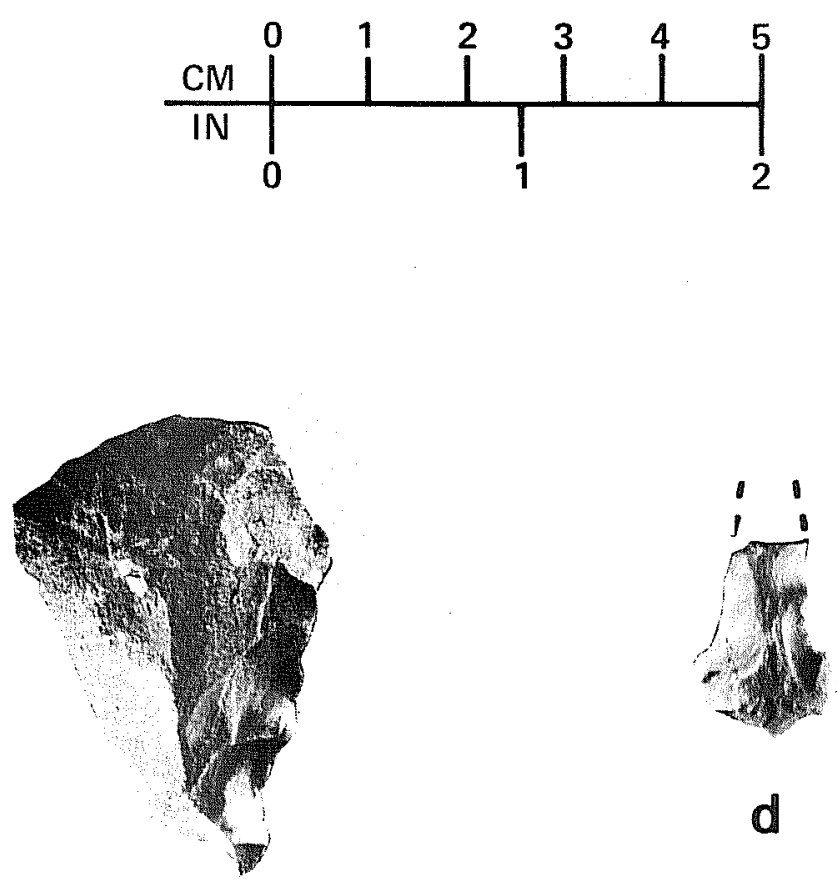

d

C

Figure 9. Chipped Stone Artifacts. a, flake with marginal unifacial retouching; $b$, flake with overall unifacial retouching; $c$, flake with marginal bifacial retouching; $d$, perforator. 


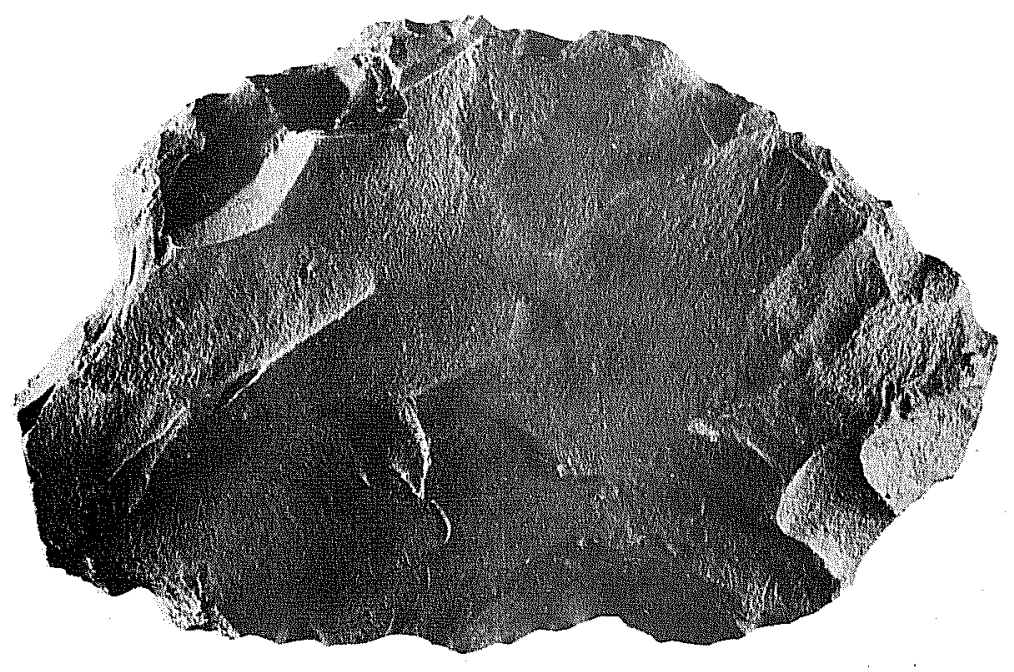

a
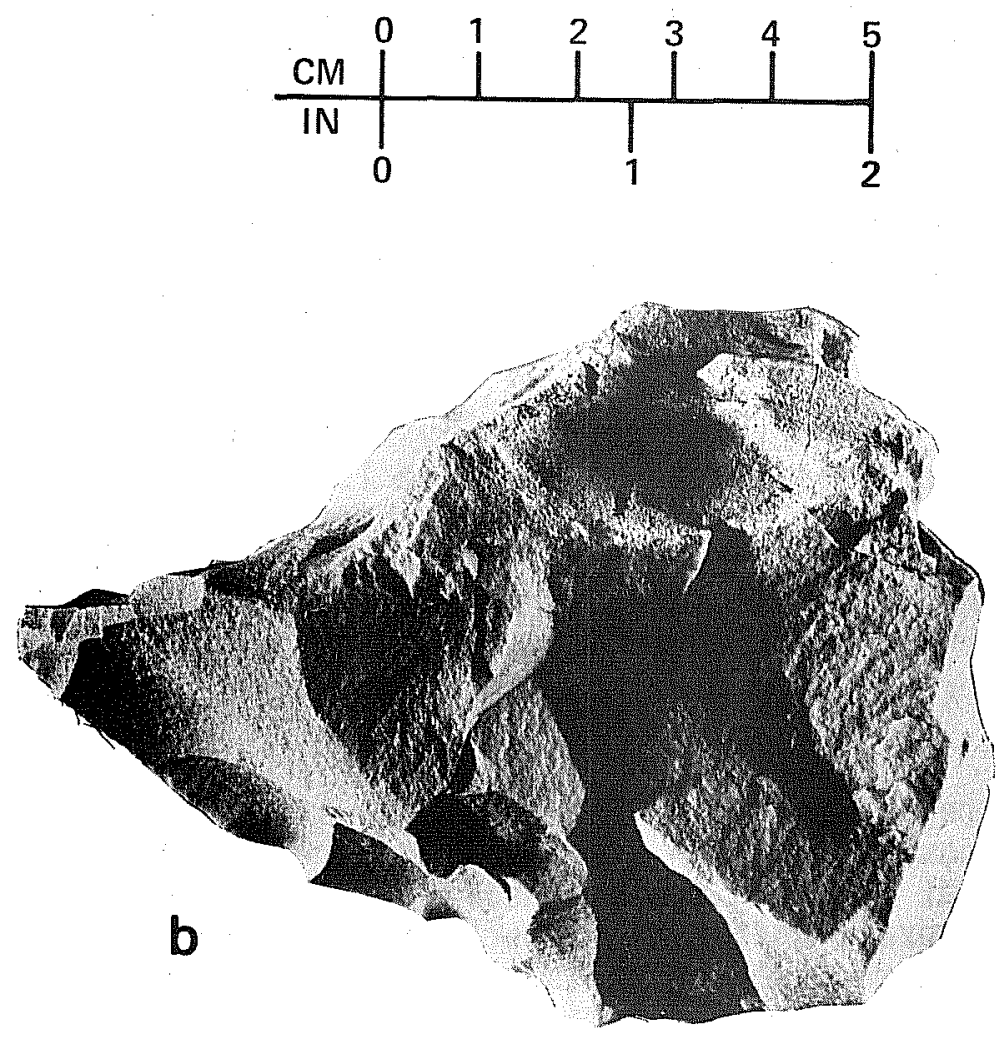

Figure 10. Chipped Stone Artifacts. $a, b$, overall unifacially retouched blank. 
The sixth and final chipped stone category is the most complex technologically and carries the most subcategories. Characteristic of all these specimens is primary bifacial overall retouching, and it is often difficult to determine from the final product whether the blank was a flake or the interior of a nodule. The small number of blanks (Fig. 11,a,b) collected are all flake blanks, however. Subsequent reduction of the blank mass and secondary marginal retouching results in a variety of tool forms: cutting tools (Fig. 11,c-e), which may also represent preforms for still further refined forms; perforators (Fig. 9,d), distinguished by their narrow, diamond-shaped blade; and projectile points (Figs. 1214), distinguishable by their blade forms and a variety of hafting modifications.

The class of rough stone artifacts has two categories: handstones and grinding slabs. While some initial shaping of these artifacts may have been carried out by percussion (a chipped stone technique), the primary modification was through abrasion due to utilization. Handstones are found in a variety of geometric shapes, from circuloid (Fig. 15,a) through ellipspoid to rectanguloid (Fig. $15, b)$, but their low frequency of occurrence precludes any interpretations as to the functional, temporal or cultural factors behind these variations in shape. Likewise, grinding slabs vary from simple tabular slabs of sandstone to wel1-formed ovoids, ellipsoids (Fig. 16) and rectanguloids. Pottery fragments are categorized as either body sherds (Fig. 17,a-c) or rim sherds (Fig. 17,d,e). The sample might also have been categorized by wares (e.g., brownware, grayware) or by surface treatment and decoration (e.g., plainware, painted wares), but its very small size argued for the simplicity of a morphological classification.

Onty two historic artifacts were recovered: a fragment of red bottle glass and a large-calibre cartridge casing dated 1918. Both specimens were recovered at $41 \mathrm{CU} 234$, and they are tabulated with pottery body sherds and projectile points, respectively.

An artifact count by category for every newly recorded site (Table 12), previously recorded site revisited in 1976 (Table 13), and collecting locality and isolated find (Table 14) is provided. As has been previously mentioned, while the unretouched flake totals are not statistically valid, they do represent a gross approximation of overall site assemblage sizes relative to each other. Since an effort was made to recover most of the retouched stone tools, however, their totals are perhaps more meaningful in attempting functional interpretations.

A glance at the category totals in Table 12 (new sites) points up some interesting data. Among those tools with steep marginal retouching on only one face, presumed to represent scraping tools, the marginally retouched flakes far outnumber those with overall unifacial retouching. But the opposite can be observed among the presumed cutting tools, where bifaces outnumber marginally retouched flakes by a similar proportion. If the functional interpretations of these morphological and technological variations are correct, then the production of scraping tools was a much more casual affair than was the manufacture of cutting tools. A similar situation may be observed at previously recorded sites (Table 13), even though these sites had been previously collected.

Another surprising observation was the number of projectile points and point fragments collected, far outnumbering any other tool subcategory. A11 but 15 of the 63 sites from which collections were made yielded at least one specimen 


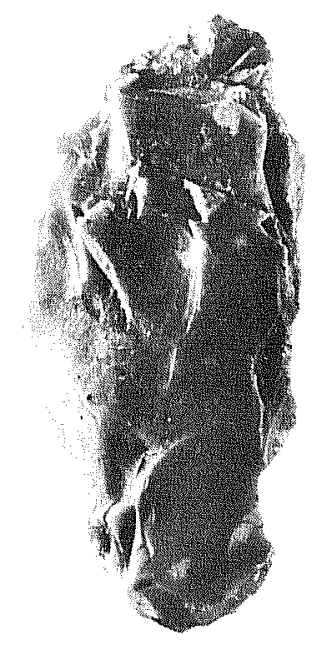

a

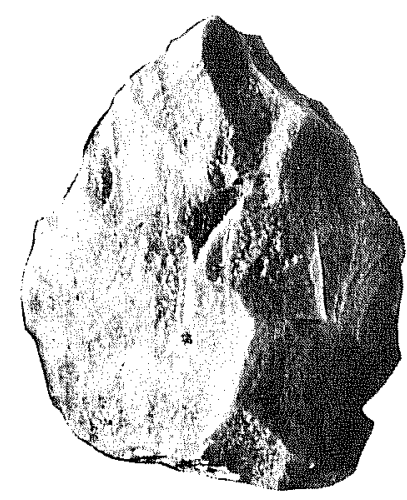

b

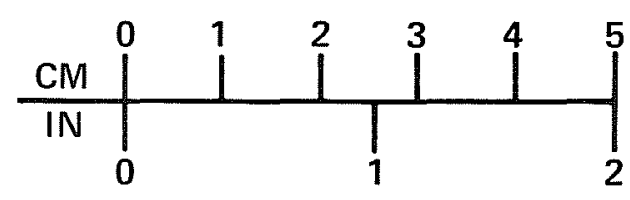

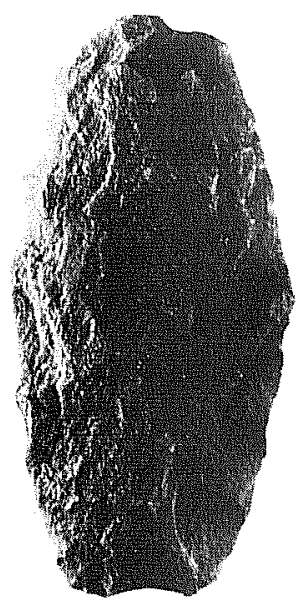

C

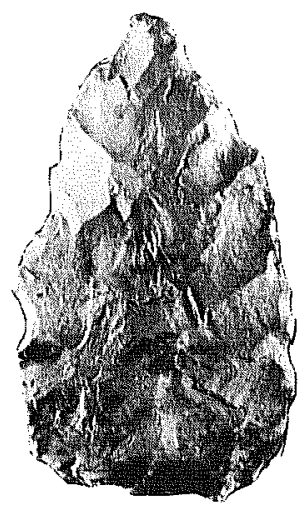

d

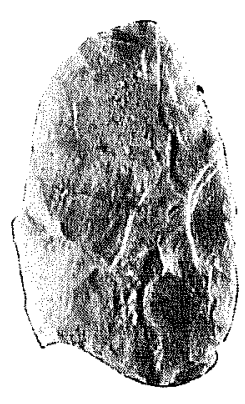

e

Figure 11. Chipped Stone Artifacts. a,b, overall bifacially retouched blank; c-e, overall bifacially retouched preform/cutting tool. 


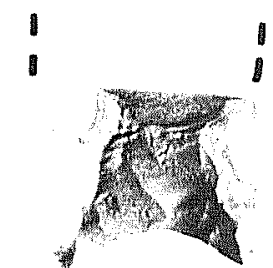

a

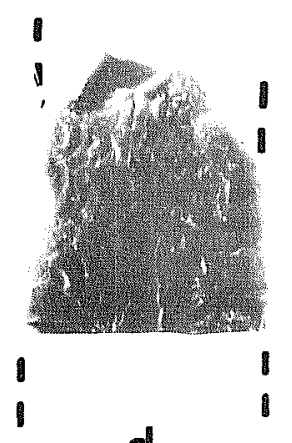

d

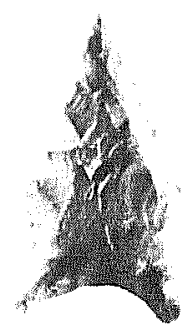

b

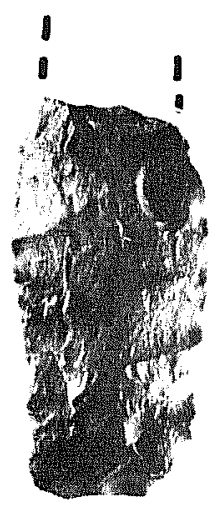

C

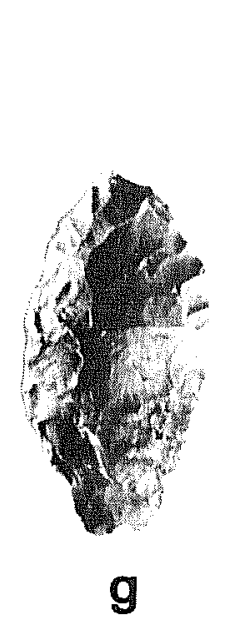

9

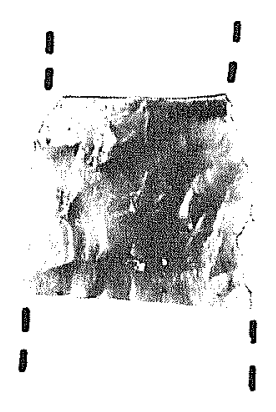

e

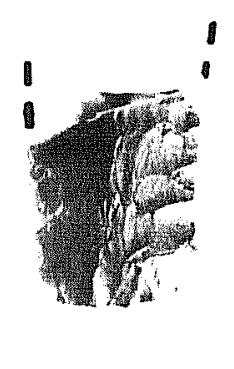

$f$
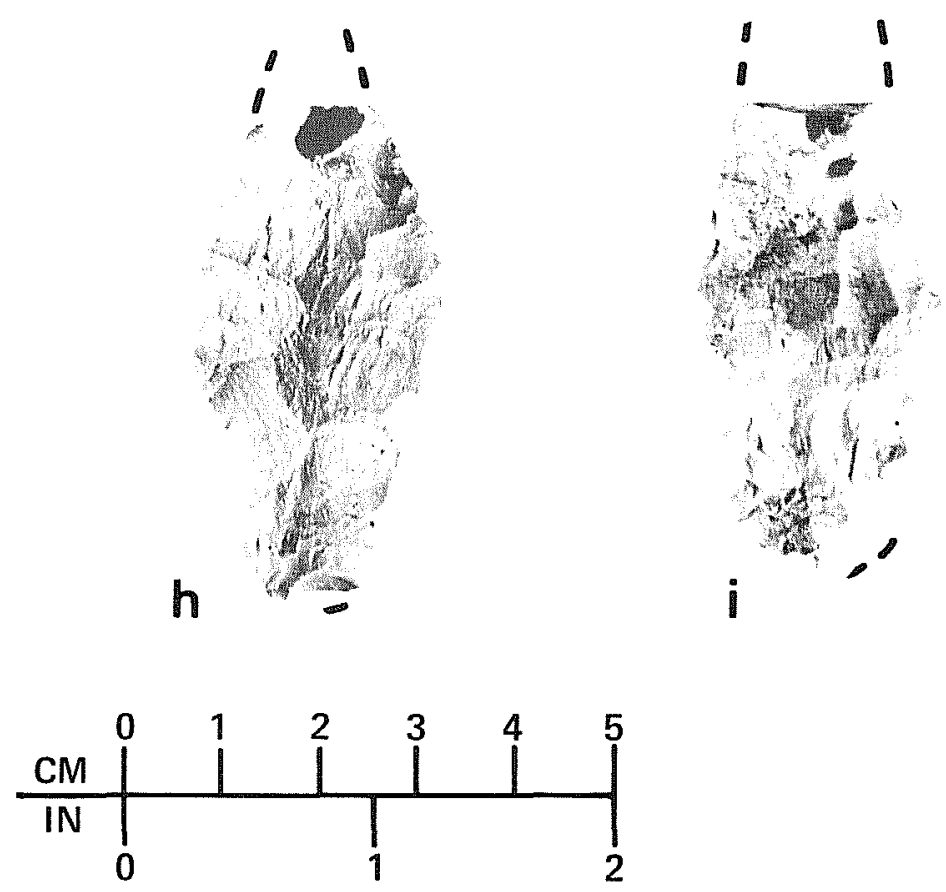

Figure 12. Projectile Points Diagnostic of the Late Paleo-Indian and Early Archaic Periods. a-f, Late Paleo-Indian (a, Plainview; b, Meserve; $c-f$, parallel-flaked blades); g-i, Early Archaic ( $g$, Pandale; $h$, Travis; i, Wells). 


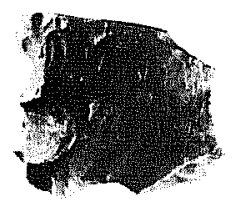

a

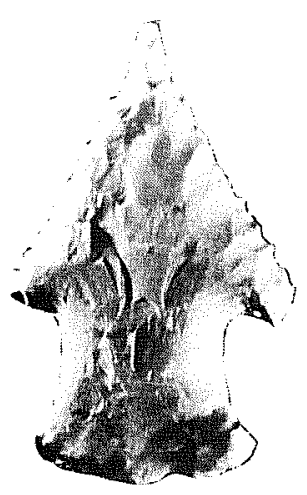

d

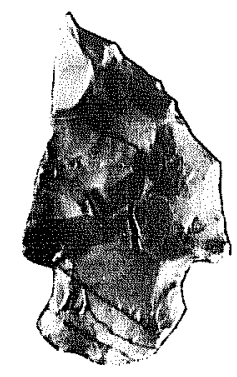

g

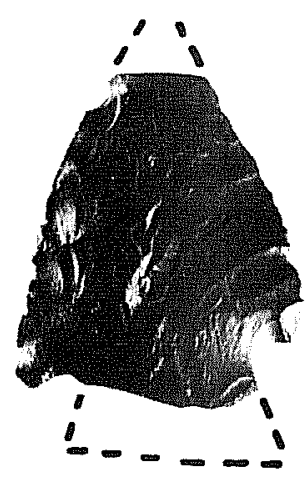

b
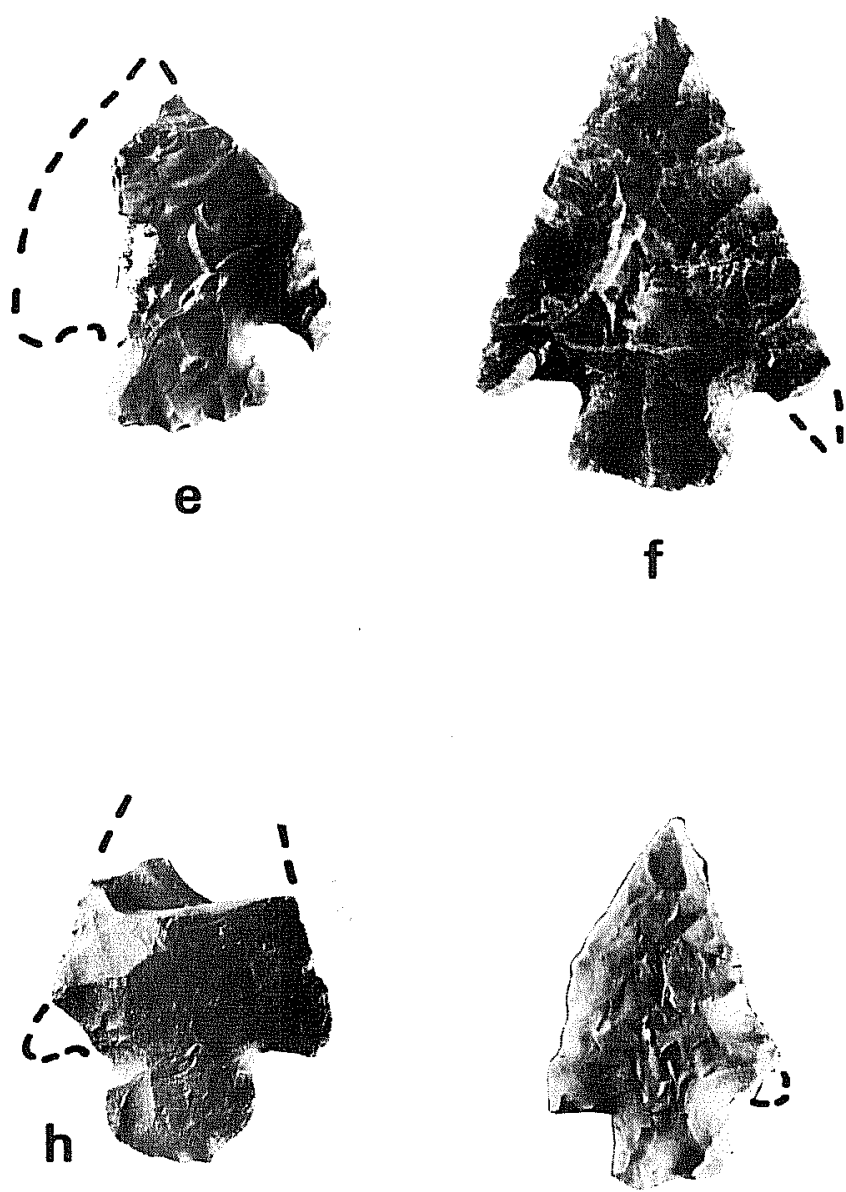

$f$

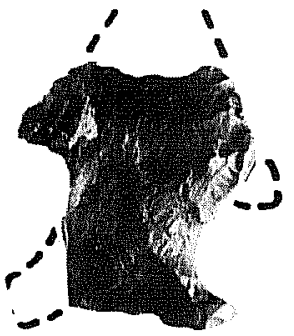

C

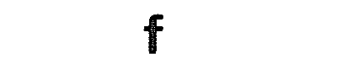

i

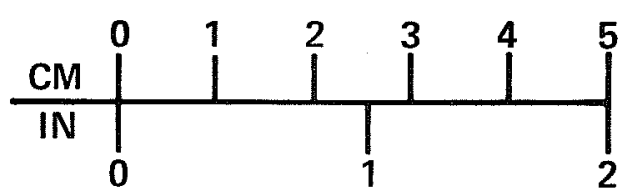

Figure 13. Projectile Points Diagnostic of the Middle and Late Archaic Periods. a, Middle Archaic (Pedernales); $b-i$, Late Archaic ( $b$, Castroville; c, Fairland; d, Lange; e, Marcos; f, Marshall; g, Palmillas; h, Williams; $i$, Yarbrough). 


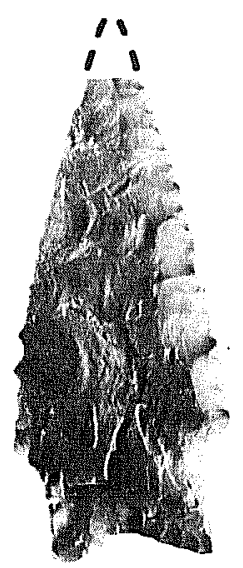

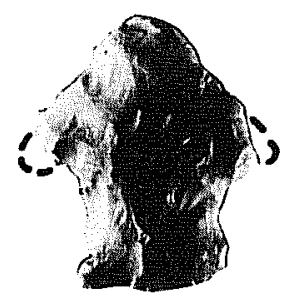

b

a

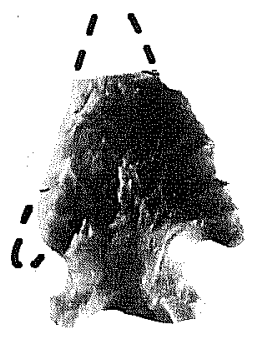

d
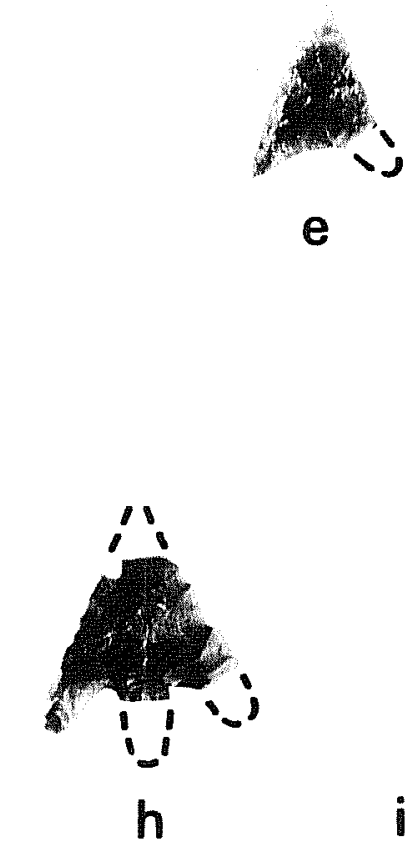

h

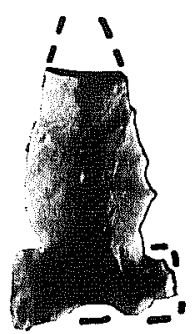

f
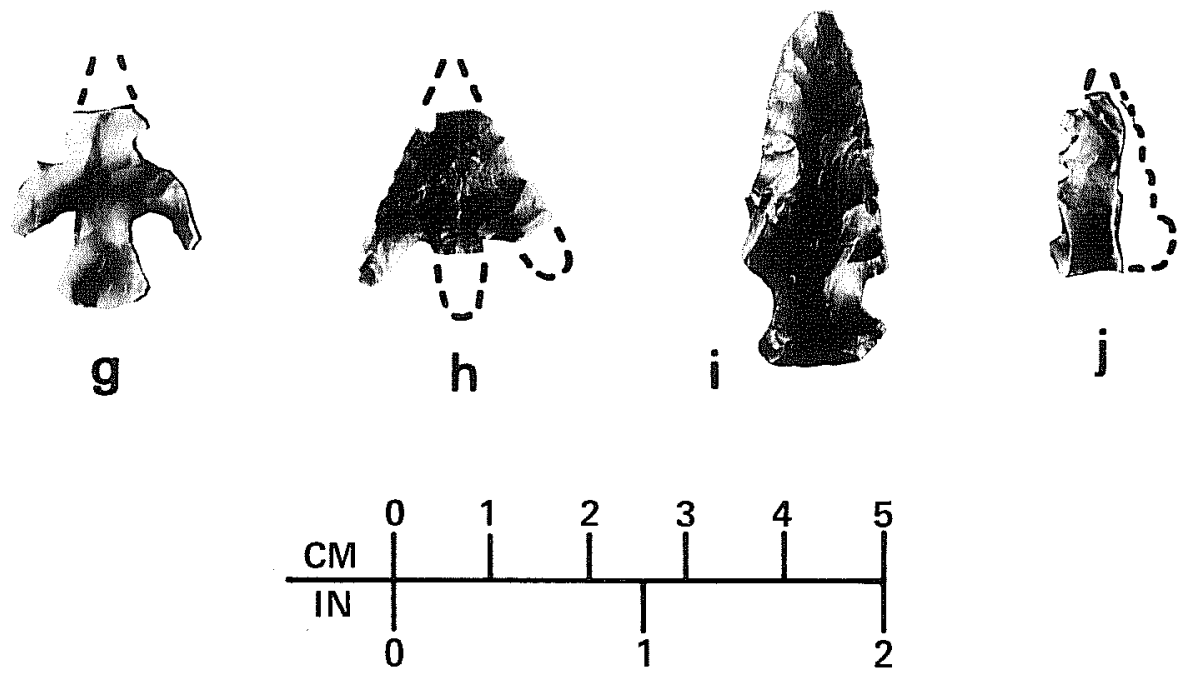

Figure 14. Projectile Points Diagnostic of the Transitional and Ceramic Periods. a-d, Transitional (a, Darl; b, Ellis; $c$, Ensor; d, Frio); e-j, Ceramic (e, Fresno; $f$, Harrell; g, Livermore; h, Perdiz; i, Scallorn; j, Toyah). 

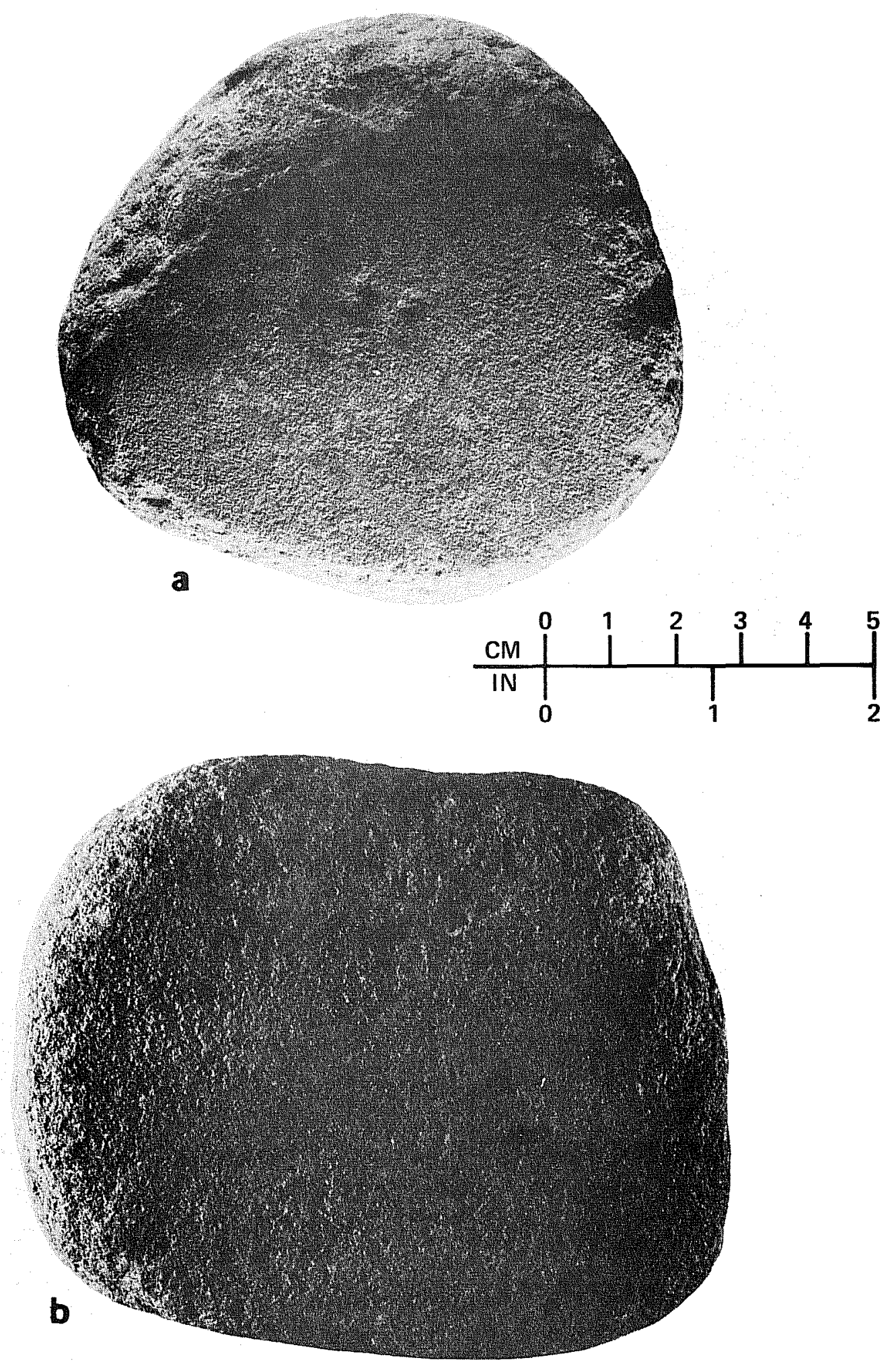

Figure 15. Handstones. a, circuloid variety; $b$, rectanguloid variety. 
17.

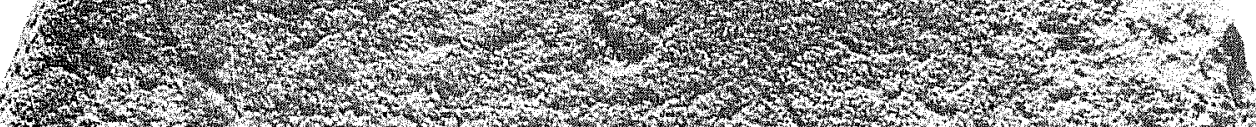

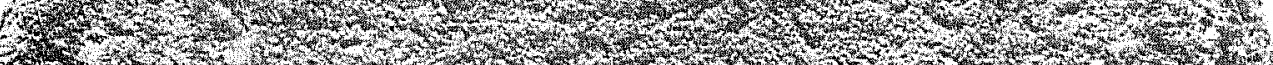

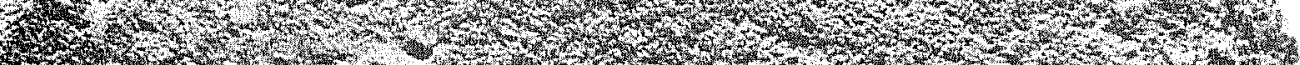

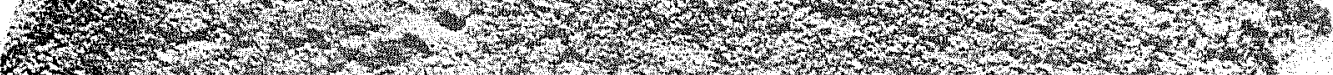

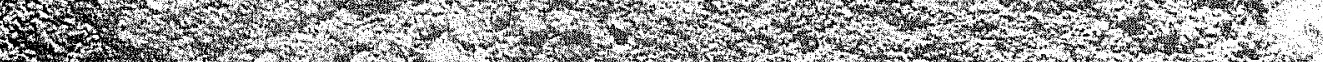
7.

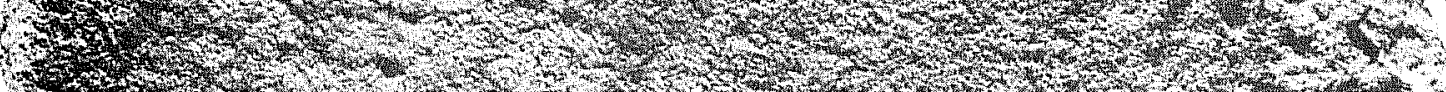

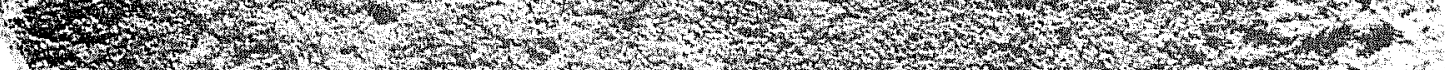

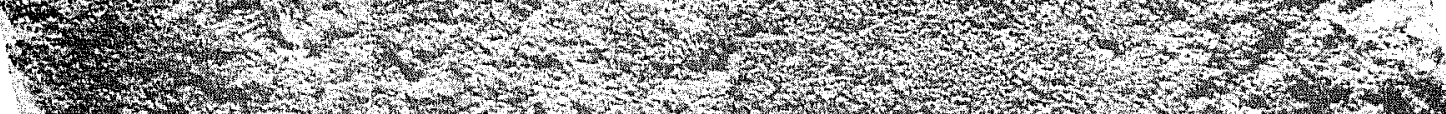

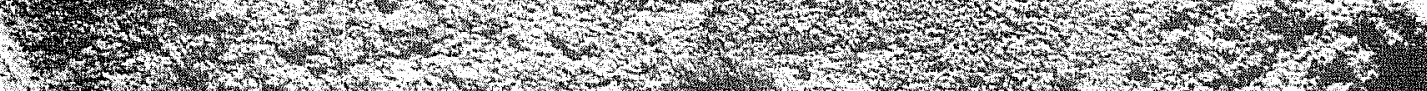

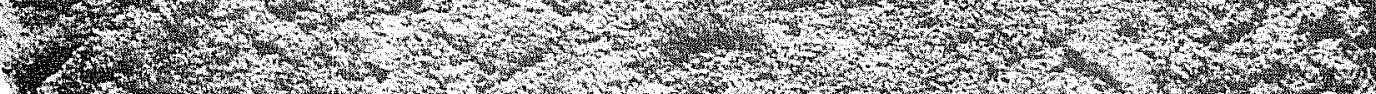

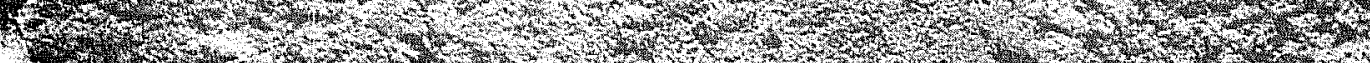
7.7.

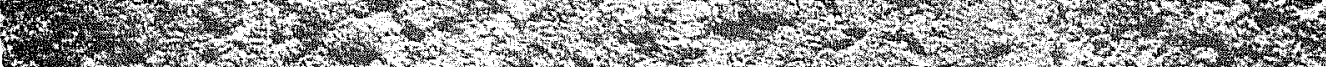

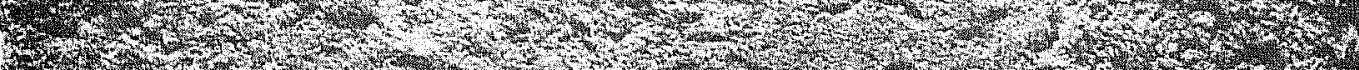
(7) 3
7

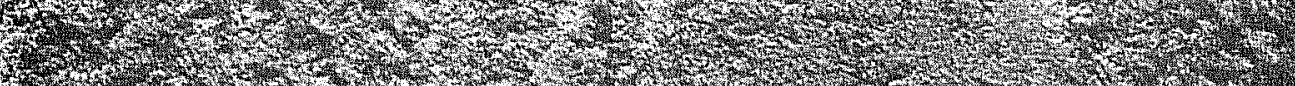

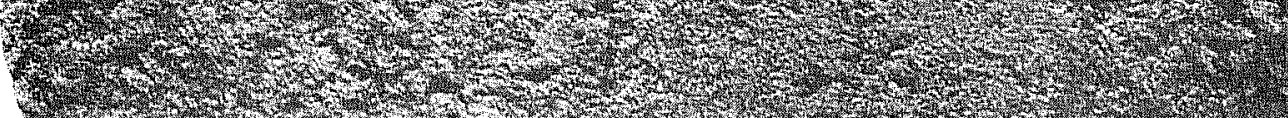

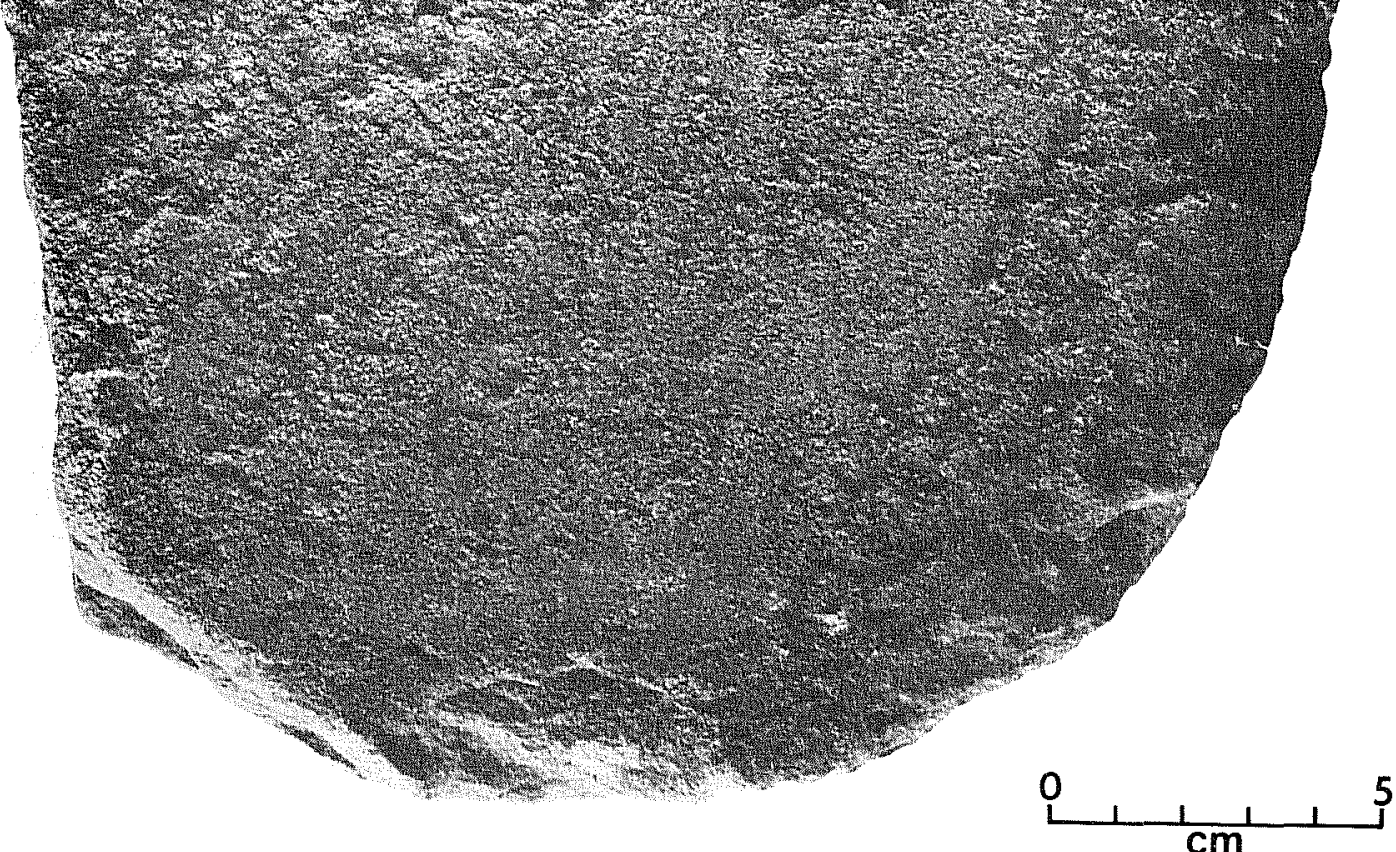

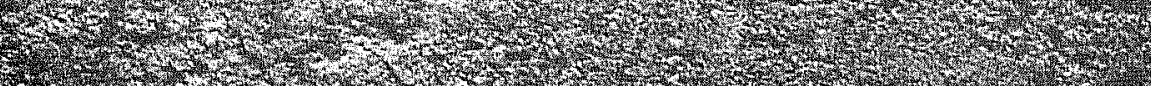

Figure 16. Grinding Slab. 

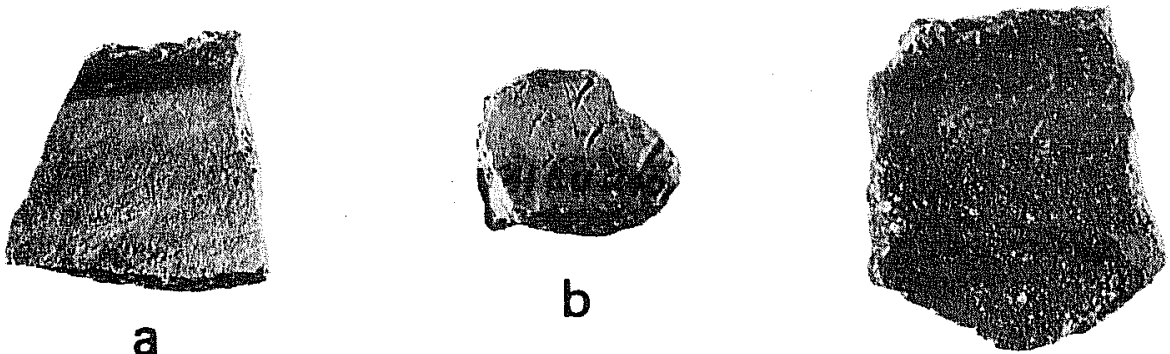

a

b

C
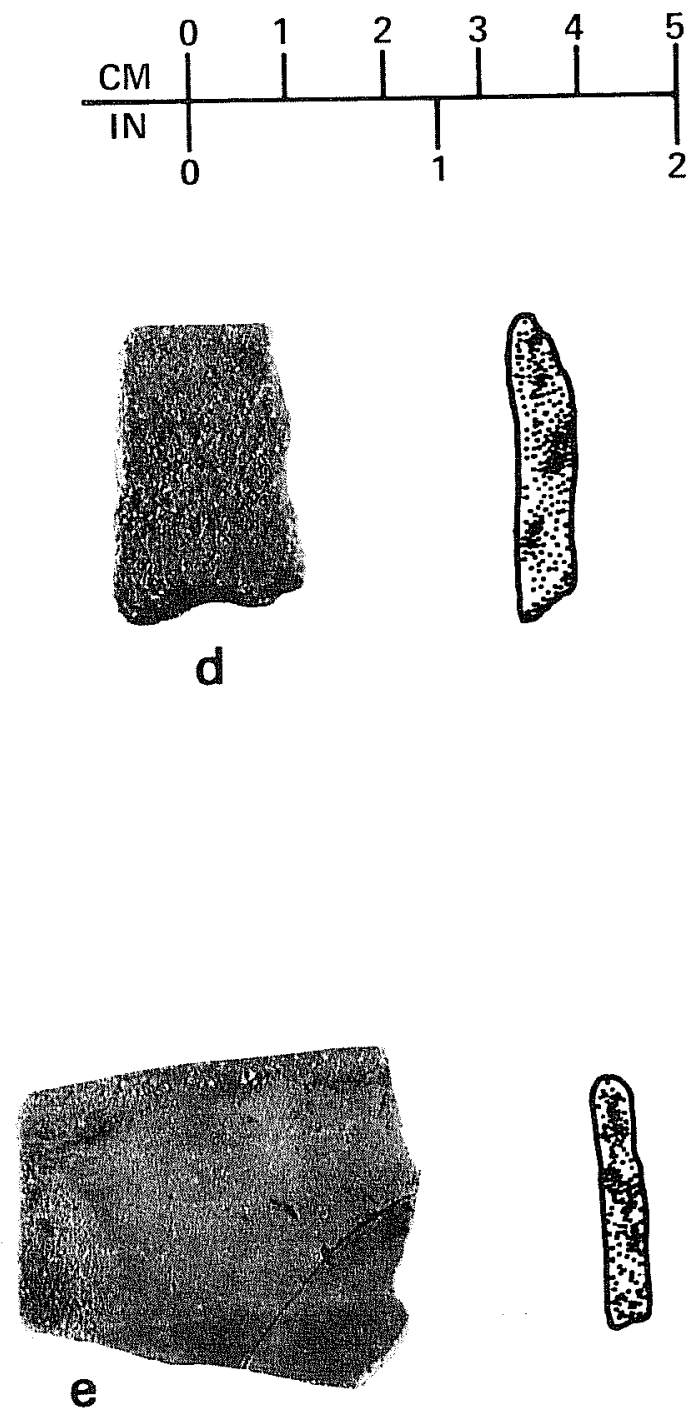

Figure 17. Representative Pottery Types Documented in the High Country in 1976. a, Chupadero Black-on-White body sherd; b, Three Rivers Red-on-Terracotta body sherd; c, El Paso Polychrome body sherd; d, El Paso Brown rim sherd; e, Jornada Brown rim sherd. 
TABLE 12. CORRELATION OF SITES WITH RECOVERED ARTIFACT CATEGORIES--NEW SITES

\begin{tabular}{|c|c|c|c|c|c|c|c|c|c|c|c|c|c|c|c|c|}
\hline & 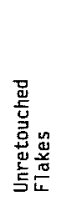 & 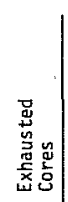 & 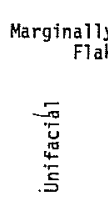 & 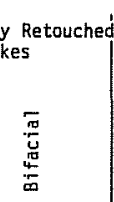 & 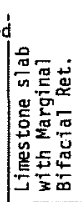 & $\begin{array}{l}\text { Unifac } \\
\text { Ret } \\
\end{array}$ & 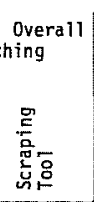 & 竘 & 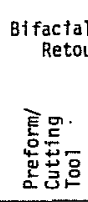 & 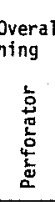 & 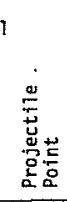 & 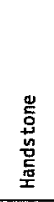 & 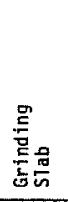 & 畣 & $\stackrel{E}{\Sigma}$ & 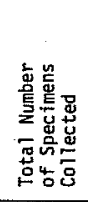 \\
\hline 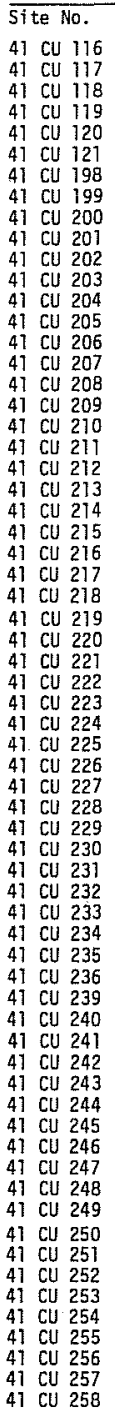 & $\begin{array}{c}28 \\
76 \\
10 \\
9 \\
13 \\
6 \\
42 \\
490 \\
190 \\
58 \\
57 \\
100 \\
8 \\
18 \\
17 \\
17 \\
22 \\
5 \\
6 \\
14 \\
34 \\
8 \\
29 \\
69 \\
95 \\
10 \\
89 \\
231 \\
33 \\
77 \\
327 \\
39 \\
19 \\
61 \\
34 \\
27 \\
7 \\
25 \\
17 \\
33 \\
155 \\
29 \\
23 \\
25 \\
148 \\
24 \\
16 \\
81 \\
51 \\
30 \\
22 \\
99 \\
31 \\
57 \\
75 \\
N 0 \\
53 \\
79 \\
23 \\
75 \\
29 \\
55 \\
123 \\
18 \\
71 \\
95\end{array}$ & $\begin{array}{l}1 \\
2\end{array}$ & $\begin{array}{c}1 \\
\\
1 \\
1 \\
2 \\
1 \\
1 \\
2 \\
\text { S LOCATED } \\
1 \\
\\
4 \\
1 \\
1 \\
2 \\
1 \\
2 \\
4 \\
6 \\
1 \\
15 \\
1 \\
1 \\
9 \\
2 \\
1 \\
3 \\
2 \\
1 \\
5 \\
1 \\
6 \\
6 \\
1 \\
2 \\
4 \\
2 \\
2 \\
3 \\
1 \\
1 \\
\\
\end{array}$ & $\begin{array}{c}1 \\
1 \\
\text { ON SURFACE } \\
1 \\
3 \\
1 \\
1 \\
1 \\
2 \\
1\end{array}$ & ${ }^{1}$ & $\begin{array}{l}1 \\
2\end{array}$ & 5 & 1 & $\begin{array}{c}3 \\
2 \\
1 \\
3 \\
\\
2 \\
1 \\
1 \\
1 \\
1 \\
2 \\
5 \\
5 \\
1 \\
20 \\
2 \\
15 \\
2 \\
2 \\
1 \\
3 \\
1 \\
1 \\
2 \\
8 \\
\\
4 \\
\\
6 \\
1 \\
1 \\
8 \\
3 \\
1 \\
1 \\
1 \\
1 \\
1 \\
2 \\
4 \\
3 \\
3\end{array}$ & $\begin{array}{l}1 \\
1\end{array}$ & $\begin{array}{c}4 \\
3 \\
\\
1 \\
2 \\
2 \\
7 \\
1 \\
2 \\
1 \\
1 \\
1 \\
1 \\
1 \\
1 \\
1 \\
2 \\
3 \\
2 \\
8 \\
1 \\
5 \\
27 \\
6 \\
1 \\
24 \\
3 \\
1 \\
4 \\
6 \\
1 \\
1 \\
1\end{array}$ & $\begin{array}{l}1 \\
2 \\
3\end{array}$ & $\begin{array}{l}2 \\
2\end{array}$ & $\begin{array}{l}25 \\
1^{*} \\
2 \\
1 \\
2 \\
2\end{array}$ & 1 & $\begin{array}{r}33 \\
83 \\
11 \\
13 \\
15 \\
8 \\
58 \\
501 \\
62 \\
66 \\
60 \\
0 \\
8 \\
22 \\
18 \\
19 \\
27 \\
5 \\
9 \\
17 \\
39 \\
14 \\
43 \\
76 \\
115 \\
13 \\
96 \\
302 \\
40 \\
82 \\
396 \\
47 \\
21 \\
74 \\
44 \\
30 \\
11 \\
32 \\
19 \\
37 \\
211 \\
34 \\
5 \\
38 \\
167 \\
27 \\
21 \\
94 \\
55 \\
32 \\
26 \\
117 \\
37 \\
60 \\
79 \\
0 \\
55 \\
84 \\
26 \\
79 \\
32 \\
69 \\
142 \\
22 \\
77 \\
122 \\
122 \\
\end{array}$ \\
\hline $\begin{array}{l}\text { Category } \\
\text { Total }\end{array}$ & 3327 & 7 & 107 & 12 & 8 & 13 & 7 & 27 & 117 & 4 & 203 & 17 & 8 & 58 & 2 & 3917 \\
\hline
\end{tabular}

* = historic artifact 
TABLE 13. CORRELATION OF SITES WITH RECOVERED ARTIFACT CATEGORIES--PREVIOUSLY RECORDED SITES REVISITED IN 1976

|
Artifact


TABLE 14. CORRELATION OF COLLECTING LOCALITIES AND ISOLATED FINDS WITH RECOVERED ARTIFACT CATEGORIES

\begin{tabular}{|c|c|c|c|c|c|c|c|c|c|c|c|c|c|c|c|c|}
\hline $\begin{array}{l}\text { Artifact } \\
\text { Categories }\end{array}$ & 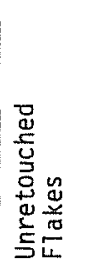 & 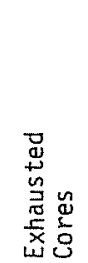 & 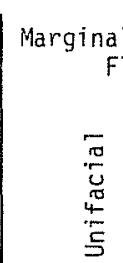 & $\begin{array}{l}\text { Retouched } \\
\text { s } \\
\bar{\sigma} \\
\frac{\pi}{4} \\
\frac{\pi}{6} \\
\bar{\infty}\end{array}$ & 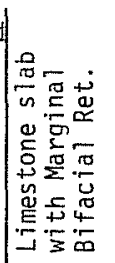 & $\begin{array}{l}\text { Unifaci } \\
\text { Reto }\end{array}$ & 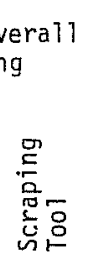 & 总 & 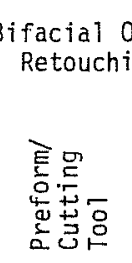 & 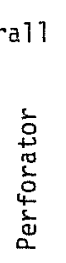 & 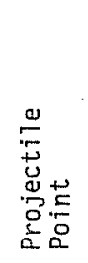 & 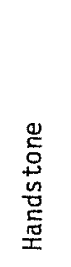 & 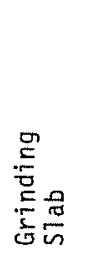 & Sher & $\underset{\square}{E}$ & 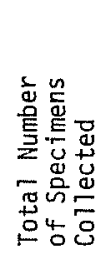 \\
\hline 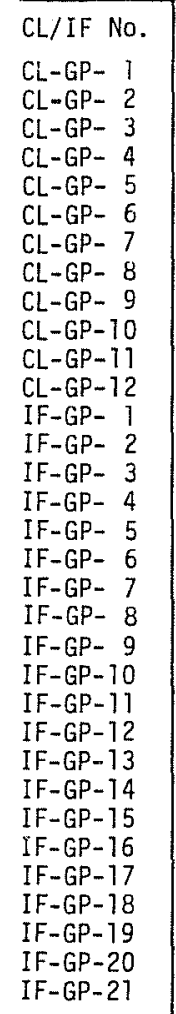 & $\begin{array}{r}25 \\
15 \\
14 \\
10 \\
2 \\
2 \\
10 \\
18 \\
6 \\
5 \\
9 \\
9 \\
9 \\
36 \\
4 \\
2 \\
1 \\
3\end{array}$ & 1 & $\begin{array}{l}2 \\
\\
2 \\
1 \\
2 \\
2 \\
1 \\
1 \\
3 \\
1 \\
6 \\
1 \\
1 \\
1\end{array}$ & 1 & & & & 1 & $\begin{array}{l}2 \\
2 \\
2 \\
1 \\
1\end{array}$ & 1 & $\begin{array}{l}2 \\
1 \\
1 \\
1 \\
1 \\
3 \\
5 \\
1 \\
1 \\
1 \\
2 \\
2 \\
1 \\
1 \\
1 \\
1 \\
1 \\
1 \\
1 \\
1 \\
1 \\
1 \\
1\end{array}$ & & & & & $\begin{array}{r}30 \\
15 \\
14 \\
12 \\
5 \\
15 \\
20 \\
10 \\
7 \\
73 \\
15 \\
15 \\
48 \\
5 \\
3 \\
4 \\
5 \\
2 \\
2 \\
1 \\
1 \\
1 \\
4 \\
2 \\
1 \\
3 \\
1 \\
2 \\
10 \\
5 \\
1 \\
1 \\
1 \\
1 \\
3\end{array}$ \\
\hline $\begin{array}{l}\text { Category } \\
\text { Totals }\end{array}$ & 189 & 2 & 27 & 1 & 0 & 0 & 0 & 2 & 10 & 1 & 30 & 0 & 0 & 0 & 0 & 262 \\
\hline
\end{tabular}


identified as a point; an average of four points was collected at each site, with a range of from one to 27. Again, the use of total count figures may mask certain functional or chronological variations between the sites, but the widespread occurrence of this particular tool throughout the High Country is undeniable. In fact, of the 13 sites yielding five or more points, nine of them are midden sites and only four are featureless lithic scatters. It is suggested that whatever other subsistence activities were being pursued, products of the hunt were always a component of aboriginal economy in the High Country.

\section{Chronologically Diagnostic Artifacts}

Projectile points and potsherds have been the traditional mainstays for assigning a surface collection (and thus the site from which it was recovered) to a chronological period, or for constructing a chronological profile of a multicomponent site. Toward this end, separate tabulations are provided which 1 ist every projectile point (Tables 15-17) and potsherd (Table 18) collected in 1976.

The subdivision of the chipped stone subcategory of projectile points into varieties was accomplished by reference to the type descriptions of Suhm and Jelks (1962). This type classification system has recently been tested and shown to exhibit a high degree of morphological and chronological accuracy. Weir's (1976a; 1976b) recent study of the Central Texas Archaic involved a reanalysis of 13,000 artifacts from 17 sites. The resultant regional phase sequence seems firmly grounded on data from controlled stratigraphic excavations, radiocarbon dates and detailed comparative analyses; the basic model has even been expanded recently with the inclusion of climatic data (Gunn and Weir 1976). One result has been a refinement of the chronological range of many projectile points from that in the type description (Weir 1976b:Table 1). The morphological and technological characteristics of points assigned to most of the type categories was also recently tested (Gunn and Prewitt 1975). The general conclusion was that the type descriptions can be used to sort collections of points, providing the analyzer pays strict attention to certain critical attributes when dealing with some of the more loosely defined groups.

Guadalupe Mountains National Park lies within the Trans-Pecos archaeological region (Lehmer 1960) and, although some of the sites studied by Weir occur near the mouth of the Pecos River, care must be exercised in extending the economic and settlement aspects of Weir's Central Texas Archaic model too far west. Since almost all of the projectile point types associated with Weir's phases are interregional in distribution, however, the chronological framework which they establish is more widely applicable. Consequently, the identifiable point types collected from the High Country have been chronologically ordered within Tables 15-17, at least to the extent that they are grouped within sequent temporal periods. The resultant chronological framework basically reflects that developed from the 1973 survey, with refinements made possible by reference to Weir's data.

Figures 12-14 illustrate each of the named projectile point types 1isted in Tables 15-17; the five varieties of hafting elements recovered and assorted blade fragments (tips and mid-sections) are listed but not illustrated. Morphological characteristics of the haft element and shoulder region were the primary sorting criteria employed to identify types and assign chronological periods, with a single exception. The presence of parallel, transverse, diagonal ribbon 
TABLE 15. CORRELATION OF PROJECTILE POINT TYPES WITH NEWLY RECORDED HIGH COUNTRY SITES

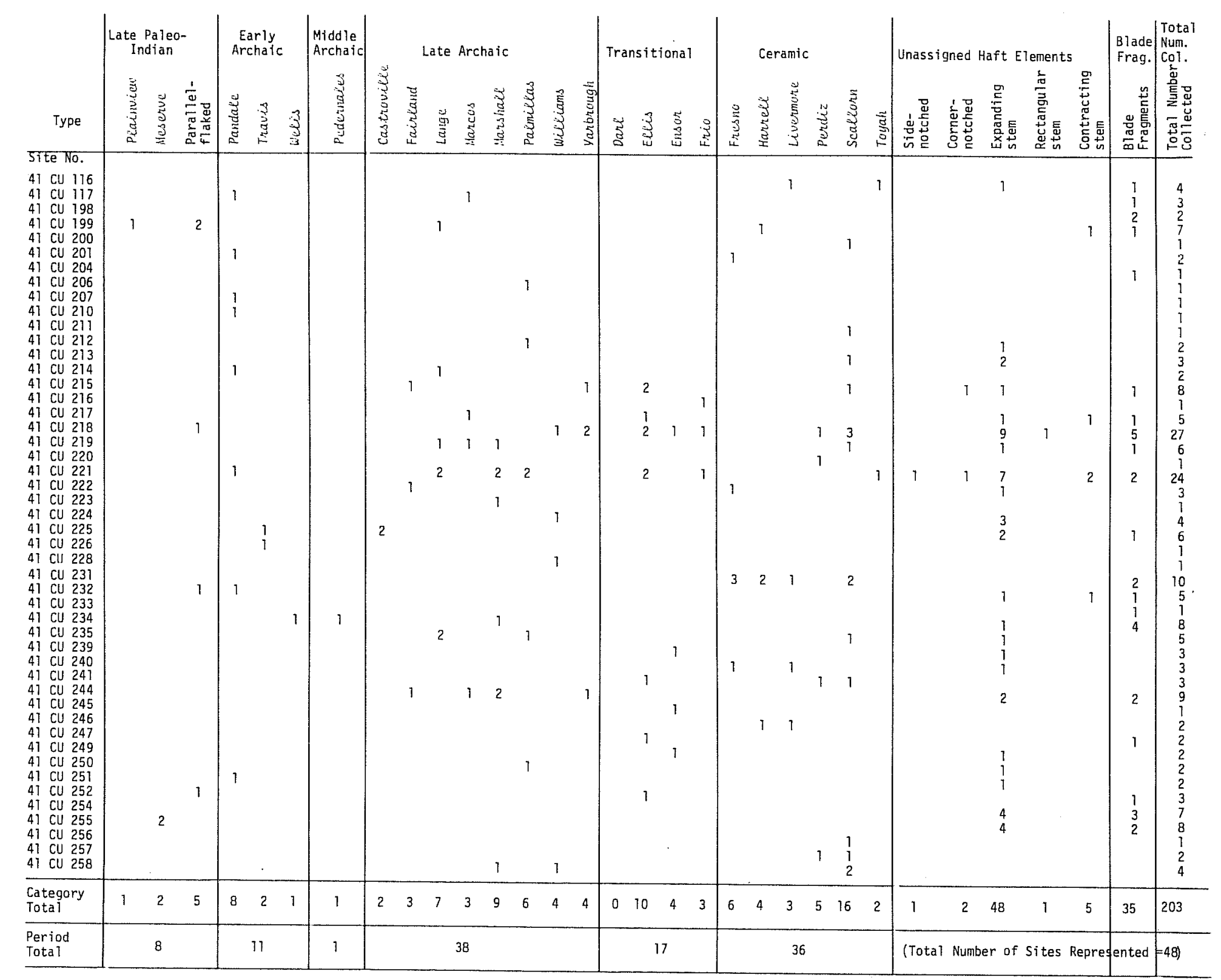


TABLE 16. CORRELATION OF PROJECTILE POINT TYPES WITH PREVIOUSLY RECORDED SITES REVISITED IN 1976

\begin{tabular}{|c|c|c|c|c|c|c|c|c|c|c|c|c|c|c|}
\hline Type & $\begin{array}{l}0 \\
\text { హ్త } \\
\text { క్త }\end{array}$ & $\frac{\$}{\frac{3}{3}}$ & $\begin{array}{l}\$ \\
\vdots \\
\vdots \\
\vdots \\
2\end{array}$ & ફે & 疋 & 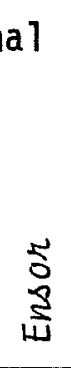 & $\begin{array}{l}\text { ఫั } \\
\vdots \\
\text { ఫ }\end{array}$ & $\begin{array}{l}\text { בे } \\
\text { క్ } \\
\text { క్ }\end{array}$ & $\begin{array}{l}\text { ָे } \\
\text { ఫ } \\
2\end{array}$ & ई & 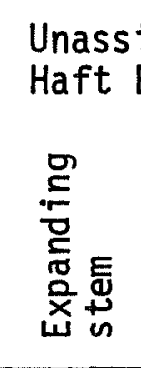 & 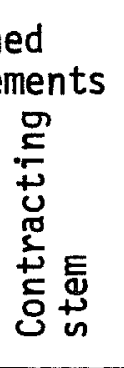 & 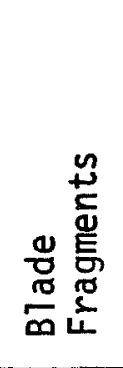 & 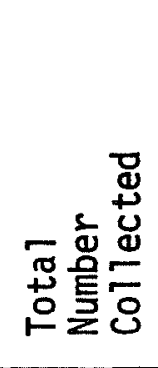 \\
\hline \multicolumn{15}{|l|}{ Site No. } \\
\hline \multicolumn{15}{|c|}{ Sites Recorded By The 1970 TAS Survey } \\
\hline $\begin{array}{lll}41 & C U & 80 \\
41 & C U & 96 * \\
41 & C U & 97 * \\
41 & C U & 98 *\end{array}$ & & & & 1 & 1 & & 1 & $\begin{array}{l}4 \\
1\end{array}$ & 2 & 1 & $\begin{array}{l}1 \\
1\end{array}$ & 1 & & $\begin{array}{l}3 \\
1 \\
9 \\
1\end{array}$ \\
\hline \multicolumn{15}{|c|}{ Sites Recorded By The 1973 TTU Survey } \\
\hline \begin{tabular}{lll||}
41 & $C U$ & 140 \\
41 & $C U$ & 169 \\
41 & $C U$ & 175 \\
41 & $C U$ & 177
\end{tabular} & 1 & 1 & 1 & & 1 & 1 & & $\begin{array}{l}2 \\
1\end{array}$ & & & $\begin{array}{l}2 \\
1\end{array}$ & 1 & $\begin{array}{l}1 \\
1\end{array}$ & $\begin{array}{l}6 \\
3 \\
2 \\
3\end{array}$ \\
\hline \multicolumn{15}{|c|}{ Sites Recorded By The 1974 TTU Field School } \\
\hline $\begin{array}{lll}41 & C U & 184 \\
41 & C U & 185 \\
\end{array}$ & 2 & & & & 1 & & & & & 1 & $\begin{array}{l}1 \\
1\end{array}$ & & 1 & $\begin{array}{l}4 \\
3 \\
\end{array}$ \\
\hline $\begin{array}{l}\text { Category } \\
\text { Tota1 }\end{array}$ & 3 & 1 & 1 & 1 & 3 & 1 & 1 & 8 & 2 & 2 & 7 & 2 & 3 & 35 \\
\hline $\begin{array}{l}\text { Period } \\
\text { Total }\end{array}$ & & 5 & & & 5 & & & & & & $\begin{array}{l}\text { (Tot } \\
\text { Num }\end{array}$ & ber & $S \mathrm{Rg}$ & $n$ ted $=10$; \\
\hline
\end{tabular}

*Not in High Country as defined 
TABLE 17. CORRELATION OF PROJECTILE. POINT TYPES WITH COLLECTING LOCALITIES AND ISOLATED FINDS

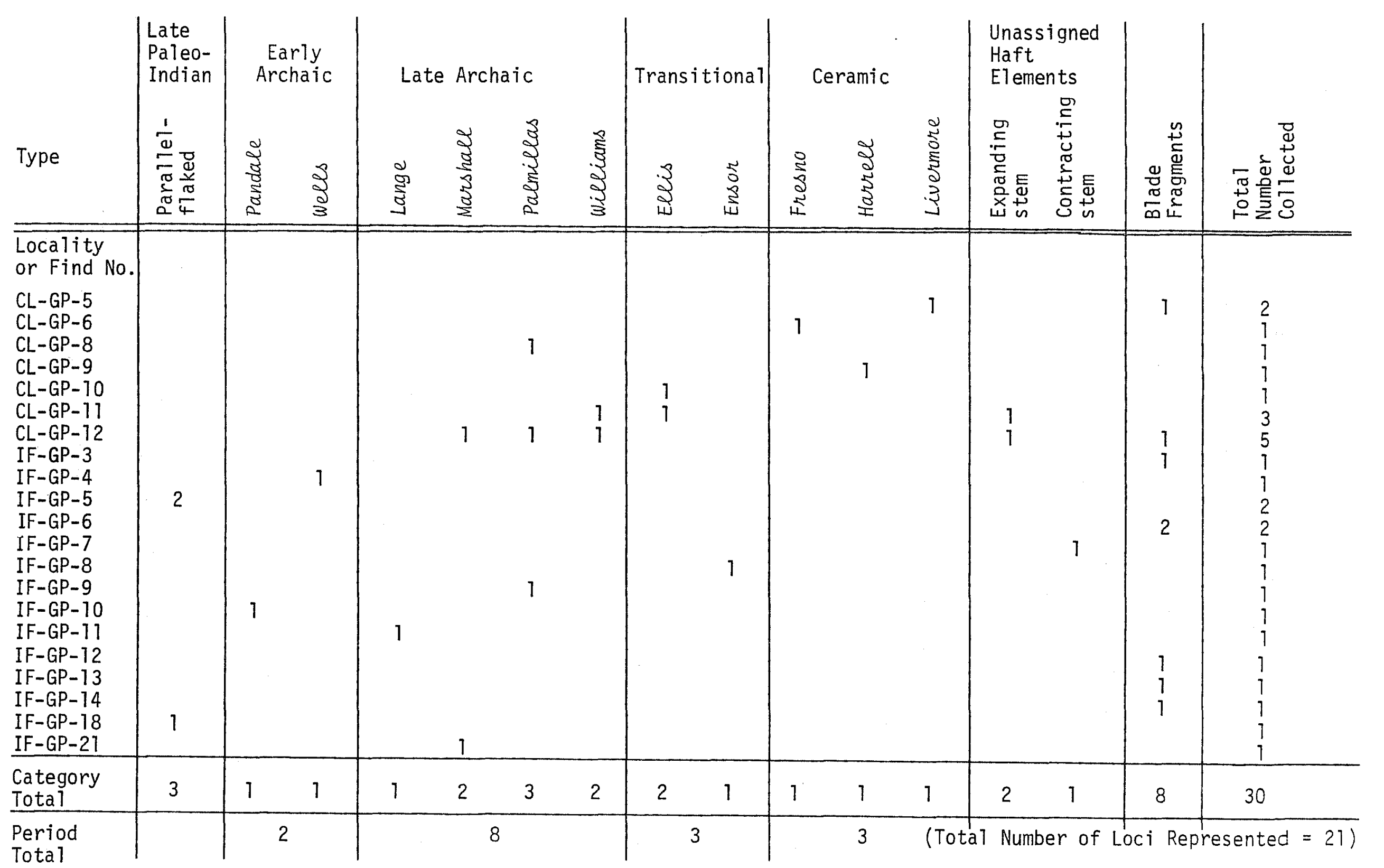


TABLE 18. SHERD CLASSIFICATION BY SITE

\begin{tabular}{|c|c|c|c|c|c|c|c|c|c|c|c|c|c|c|c|c|c|}
\hline \multirow[b]{3}{*}{ Site No. } & \multicolumn{12}{|c|}{ Newly Recorded Sites } & \multicolumn{5}{|c|}{ Previously Recorded Sites } \\
\hline & \multirow[b]{2}{*}{117} & \multirow[b]{2}{*}{209} & \multirow[b]{2}{*}{213} & \multirow[b]{2}{*}{221} & \multirow[b]{2}{*}{225} & \multirow[b]{2}{*}{231} & \multirow[b]{2}{*}{234} & \multirow[b]{2}{*}{236} & \multirow[b]{2}{*}{240} & \multirow[b]{2}{*}{243} & \multirow[b]{2}{*}{245} & \multirow[b]{2}{*}{258} & \multicolumn{3}{|c|}{1970 TAS } & \multicolumn{2}{|c|}{1973 TTU } \\
\hline & & & & & & & & & & & & & $96 *$ & $97 *$ & $98 *$ & 169 & 185 \\
\hline $\begin{array}{l}\text { Pottery } \\
\text { Classification }\end{array}$ & & & & & & & & & & & & & & & & & \\
\hline $\begin{array}{l}\text { Jornada } \\
\text { Brown }\end{array}$ & 2 & & 3 & 2 & 1 & $7^{(1)}$ & & & & & 2 & 5 & 2 & 8 & 5 & & \\
\hline $\begin{array}{l}\text { El Paso } \\
\text { Brown }\end{array}$ & & 2 & & 1 & & 15 & & & & 2 & & $7^{(1)}$ & & $23^{(3)}$ & & & 3 \\
\hline $\begin{array}{l}\text { Unidentifiable } \\
\text { Brownware }\end{array}$ & & & & 1 & & & & & & & & & & 2 & & & \\
\hline $\begin{array}{l}\text { El Paso } \\
\text { Polychrome }\end{array}$ & & & & & & & & 1 & & & & & & $6^{(1)}$ & $2^{(1)}$ & & \\
\hline $\begin{array}{l}\text { Three Rivers } \\
\text { Red/Terracotta }\end{array}$ & & & & & & & & & 1 & & & 1 & & & & & \\
\hline $\begin{array}{l}\text { Chupadero } \\
\text { Black/white }\end{array}$ & & & & & & 2 & & & & & & & & 2 & & 1 & 1 \\
\hline $\begin{array}{l}\text { Unidentifiable } \\
\text { Grayware }\end{array}$ & & & & 1 & & 2 & & & & & & & & & & & \\
\hline $\begin{array}{l}\text { Unidentifiable } \\
\text { Ware }\end{array}$ & & & & & & & & 1 & & & & & & & & & \\
\hline Historic Glass & & & & & & & 1 & & & & & & & & & & \\
\hline $\begin{array}{l}\text { Total Number } \\
\text { Collected }\end{array}$ & 2 & 2 & 3 & 5 & 1 & 26 & 1 & 2 & 1 & 2 & 2 & 13 & 2 & 41 & 7 & 1 & 4 \\
\hline
\end{tabular}


flake scars on the blade element of a large point was considered diagnostic of the Late Paleo-Indian period, even if a specific type name could not be applied.

The sherd collection from the High Country survey is quite sma11, with 60 specimens recovered from newly recorded sites and an additional five from previous ly recorded High Country sites (Table 18). Typological identification was limited to only the most obvious and commonly occurring types, due to the small size of the sherds, their often eroded condition and the absence of an adequately-sized comparative collection.

The five identifiable pottery types are those most commonly recovered from lower altitudes within the Park itself (S. Katz and P. Katz 1974; Phelps 1974) and from localities completely surrounding the Park (Applegarth 1976; Corley 1965; Henderson 1976; Katz and Lukowski n.d.; Whalen 1977). These include: Jornada Brown (Fig. 17,e); El Paso Brown (Fig. 17,d); El Paso Polychrome (Fig. 17, C); Chupadero Black-on-White (Fig. 17,a); and Three Rivers Red-on-Terracotta (Fig. 17,b). One unidentifiable brownware and three unidentifiable grayware sherds might represent any of several regional types (e.g., Roswell Brown or Crosby $B / W)$; one sherd was so carbonized that it could only be identified as pottery by virtue of visible tempering particles.

\section{DISCUSSION}

The extent to which projectile points will be used to construct patterns and make interpretations of time, space and prehistoric behavior deserves comment. Perhaps in no other part of the state could one rely as heavily on their presence and in situ recovery as is possible in the Guadalupe Mountains. Between the difficulty of gaining access to the High Country on purely topographic grounds and the fact that it was we11-protected private property prior to becoming a National Park, the incidence of relic collecting is truly negligible. A number of extensive private collections exist in the area, but the material was collected at lower elevations. Not one of the local residents with whom we talked had even been in the High Country in their lives.

To illustrate the above point, a comparison between the 1973 and 1976 collections is instructive. In 1973, 85 projectile points out of 8,011 artifacts were collected, or $1.7 \%$ of this assemblage; in 1976 the percentage was 5.2 , with 203 points out of 3,917 artifacts recovered. Even if the High Country bore more than its share of the hunting relative to other portions of the Park, we suspect that one-fifth the proportion of points elsewhere in the Park reflects a collecting bias due to relic hunting in the lower elevations.

\section{Tempora] Aspects}

Subsequent to the development of a chronological framework for Guadalupe Mountains National Park (S. Katz and P. Katz 1974:Table 4), other researchers in the region have provided their own schemes. Applegarth (1976:164), for example, feels it "is possible to identify, however tentatively, four distinct cultures and occupations of the region, "which "are separable in terms of time and content." These are designated Paleo-Indian, Archaic, the "Mogollon" and Apache. Beckett, Strong and Strong (1977) organize their prehistoric sequence according to the 
developmental stages of Willey and Phillips (1958), i.e., Lithic, Archaic and Formative Stages. The nature of the discussion indicates, however, that historical periods are intended. Henderson's (1976) scheme involves five periods, four of which concern the aboriginal populations in his locality: PaleoIndian, Archaic, Ceramic, Historic Aboriginal and Anglo-American.

While no two researchers in this region employ the same temporal scheme, none are in conflict with the sequence of periods suggested for the Park: PaleoIndian, Archaic, Transitional, Ceramic and Historic. In fact, the postulated Transitional period represents a refinement lacking in the above examples, intended to recognize a period of time during which new influences (midden features, ceramics, perhaps the bow and arrow) and possibly new peoples first began appearing in the locality.

The addition of High Country data to that collected at lower altitudes within the Park in no way alters the basic framework. The modifications that are suggested represent inter-period refinements, which now need to be tested with a reanalys is of previously collected data. Figure 18 presents a visual display of the High Country chronological framework. Each of the periods and subperiods represented can be documented by appropriate diagnostic artifacts recovered from the High Country; the date ranges follow those previously established, with substantiation provided by recent independent research.

The Paleo-Indian period is represented in the High Country only during its later portion. The basis for this determination is the recovery of Plainview, Meserve and parallel-flaked points and the failure to document clovis or Folsom projectile points. The very real problem of establishing the presence or absence of a group of people in a locality solely on the basis of accidental 1oss or discard of a particular artifact is pointed up by the recovery of several Folsom points from the Park's west side. Early Paleo-Indian activity in the High Country can thus be extrapolated, but at present it cannot be documented.

The long Archaic period has been subdivided into at least two subperiods (Early and Late), with a Middle Archaic tentatively suggested. Again, projectile point types provided the primary criteria. Suhm and Jelks (1962) were the principal source for identification of all points, but Weir $(1976 a, b)$ was heavily relied upon for refinements specific to the Archaic period. Twelve distinct projectile point types are associated with this general period (Table 15). While this is significantiy larger than the number associated with any other period, the time span of the Archaic is comparably longer.

The projectile point types associated with the Transitional period in the Park are the very ones assigned by Weir $(1976 a, b)$ to the final phase of the Central Texas Archaic (i.e., Twin Sisters): Dare, Ellis, Ensor and Frio. The 1973 data used to establish this period was reinforced by the 1976 data. The size of Transitional points is smaller than that of the previous Late Archaic period, suggesting technological differences, if not cultural ones. The postulated introduction of ceramics into the Park area during this period is supported by the presence of pottery at two of the 11 sites with Transitional point types, but only the undecorated brownware that precedes the painted varieties characteristic of the subsequent Ceramic period. Nine of these 11 sites have midden features present, moreover, and five of the nine are single feature sites. No midden mounds have been identified at any site from which Transitional points 


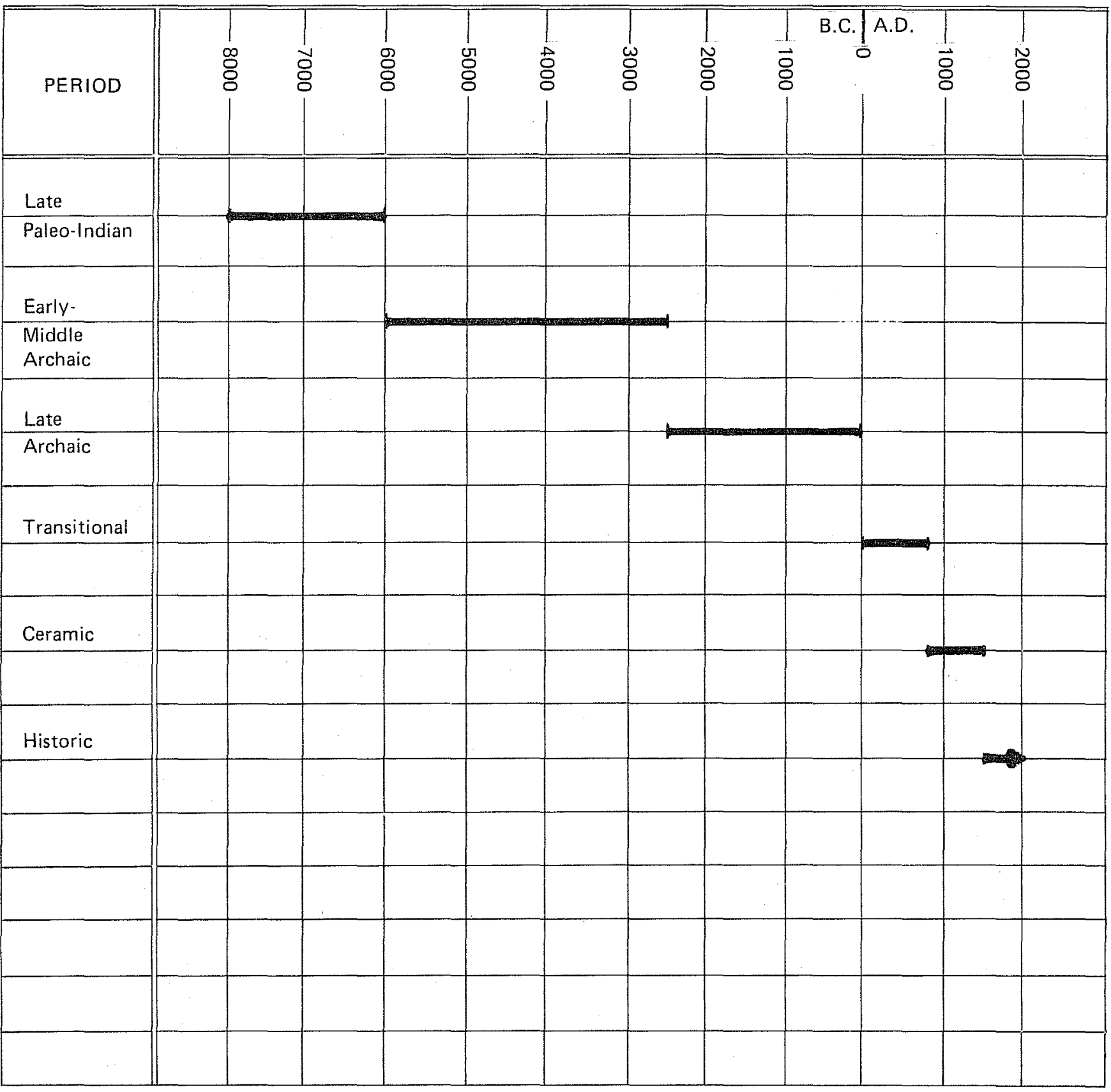

Figure 18. Chronological Framework for the High Country. 
were collected. The date range for this period of from A.D. 1 to 800 is now substantiated by the recent publication of radiocarbon dates from a Mesilla phase pithouse containing El Paso Brown sherds (Whalen 1977:Fig. B,6). The three dates have a total range of from A.D. 450 to 950 , with a mean of A.D. 640 .

The Ceramic period is represented by a variety of pottery types, with painted wares present for the first time. The associated projectile point types are the sma 11, triangular "arrow points" diagnostic of the Late Prehistoric period in other regions of Texas and the Southwest. Fourteen of the 20 sites from which Ceramic points were recovered have midden features present, and two of the three sites with midden mounds are included among them. Middens are present at nine of the 11 sites from which pottery was recovered; the exceptions include a Tithic scatter (41 CU 240) and one of the two rockshelters documented (41 CU 236).

Schemes for subdividing the Ceramic period exhibit much greater diversity than was the case for the Archaic period, primarily due to the resolution provided by ceramic attributes. The EI Paso local phase sequence established by Lehmer (1948) continues in use today (e.g., Hammersen 1972; Whaten 1977), consisting of three phases designated Mesilla, Doña Ana and El Paso. Jelinek's work (1967) in the middle Pecos Valley to the northeast resulted in the establishment of the Crosby and Roswell phases: Corley's (1965) extension of the Jornada occupation across the Pecos necessitated a new three-phase scheme based on local ceramic variations and a slight time lag. His Querecho, Maljamar and ochoa phases are dated about 50 years later than the corresponding Mesilla, Doña Ana and El Paso phases farther west.

For reasons of proximity and similarities between ceramic assemblages, the ET Paso local phase sequence is most appropriate for Guadalupe Mountains National Park. The Mesilla phase would correspond to the latter part of the Transitional period and continue into the early Ceramic period. The end of the El Paso phase and the Ceramic period are probably synchronous.

Henderson's (1976) "Historic Aboriginal" period certainly applies to the Park, although artifactual evidence is presently limited to a small number of Apachean sherds recovered at lower altitudes (Helene Warren, personal communication). Historical accounts attest to the presence of Mescalero and probably other Apachean groups in the Guadalupe Mountains (Levy 1971); one informant states that Apaches occupied site 41 CU 140 in the High Country in 1911 (Sam Hughes, Sr., as told to Roger Reisch, personal communication). To date, all researchers agree that Apachean sites are present in the southern Guadalupe Mountains, but not one such site has been positively identified. It can only be assumed that Apachean sites are very similar to Ceramic period sites and as such have been misidentified, or rather remain unidentified. A great deal of archaeological and ethnographic research will be necessary to reduce these two similar occupations to their distinguishing characteristics, research currently being undertaken by Susanna R. Katz as part of her doctoral dissertation.

Spatial Aspects

The assignment of specific sites to one or more chronological periods is usually based on the presence at the site of temporally diagnostic artifacts. Without 
the benefit of absolute dates for a particular site, however, one is never certain whether a diagnostic artifact might not have been lost within a site perimeter at a time other than that at which the site was active. This is especially critical when artifacts representative of more than one period are recovered, for the tendency in this case is to apply the designation "multicomponent" and to search for those factors which might account for the site's continual popularity. The problem becomes one of degree, of deciding how many artifacts diagnostic of a specific temporal period must be present for that site to be "dated" in this manner.

There is an intermediate step which can be taken, however, involving considerations of territory rather than specific site locations. By observing the distributions of sites having artifacts assigned to different periods, it is possible to identify variations in territories and changes in focal points for each temporal period. For example, Late Paleom Indian activity in the High Country seems to have been limited to the western third, covering an area from the BTue Ridge east to the head of South McKittrick Canyon (Fig. 1). No focus of activity is apparent, as the two sites (41 CU 199 and 255) with multiple Late Paleo-Indian points associated are situated at either side of the proposed territory (Fig。2).

Continuing to correlate point/site data (Table 15) with site locations (Fig. 2), it can be seen that Early Archaic points are distributed all over the High Country. Sites located at the limits of this extensive territory include: 41 CU 251 in the north; 41 CU 201 in the east; 41 CU 117 along the southeastern escarpment; 41 CU 234 in the Bowl; and 41 CU 226 on the Guadalupe Ridge. No focus of activity can be determined, in that none of the sites considered is characterized by more than a single point.

Only 41 CU 234 yieided a projectile point identifiable as a type assigned to the Middle Archaic period. Territorial utilization cannot be determined on the basis of such insufficient data; based on the relative number of points recovered for the various periods, it would seem that Middle Archaic activity is the most poorly represented in the High Country.

Late Archaic points exhibit an even greater spatial distribution than was observed during the Early Archaic. Diagnostic specimens extend from 41 CU 199 and 228 in the west to 41 CU 235 atop Wilderness Ridge in the far northeast corner of the Park. The clustering about the head of South McKittrick Canyon has become much more obvious, in that Late Archaic points were collected at 12 of the 19 sites associated with this locale. Five of these 12 sites, moreover, were characterized by multiple points. The only site which might be considered as single component Late Archaic, however, is 41 CU 244, located along the north arm of Devils Den Canyon. The previously recorded $41 \mathrm{CU}$ 140, located along the south arm of Devils Den Canyon, was also a multiple Late Archaic point site.

Utilization of the Devils Den Canyon area appears to increase during the Transitional period, with five sites associated. Correspondingly, the South McKittrick Canyon cluster decreases from 12 sites in the Late Archaic to 4 in the Transitional, although three of the four are multiple point sites suggesting definite occupation. Only two outliers are represented, 41 CU 252 in the north and $41 \mathrm{CU}$ 239 in the south. 
Campground trail, is the densest cluster, with 10 sites. Three of them (41 CU 218,231 and 258) have more than one point associated; 41 CU 231 is considered a single component Ceramic site based on the absence of points from other periods. The head of Devils Den Canyon and its two arms are represented by four sites, three of them with multiple Ceramic period points. A third area is the Bowl and that area known as the McKittrick Canyon Overlook, lying between the eastern escarpment and the south wall of South McKittrick Canyon. Four sites, two with multiple points, are located here. The neighboring 41 CU 199 and 200, situated along the Blue Ridge between Bush Mountain and Cox Tank, make up the fourth and last cluster of sites with Ceramic points.

Sites from which pottery was recovered reflect the three primary Ceramic point clusters: Devils Den, South McKittrick and the Bowl/Mckittrick Overlook area. If only painted sherd distribution is observed (thus factoring out the postulated Transitional period/brownware pottery association), four sites are applicable: 41 CU 240, a multiple Ceramic point site at the head of Devils Den Canyon; 41 CU 236, a neighbor of the multiple Ceramic point 41 CU 116 in the McKittrick Overlook area; the multiple Ceramic $41 \mathrm{CU} 258$ at the head of south McKittrick Canyon; and the postulated single component Ceramic 41 CU 231, also in the South McKittrick cluster.

If the recovery of temporally diagnostic projectile points is employed to establish territories of activity rather than to "date" specific sites, certain patterns are apparent and change through time. Late Paleo-Indian interests seem to be limited to the western third (eight points at five sites), while the Early Archaic manifestations are lightly scattered throughout most of the High Country (11 points at 11 sites). During the Late Archaic period, even more territory seems to have been utilized in comparison with the Early Archaic distribution, while at the same time an obvious clustering of associated sites develops for the first time (38 points at 20 sites, seven of which have more than one point). Transitional period territorial interests apparently focus on only two specific areas, with the western third of the High Country not included at al1 among the 11 sites (with 17 points) considered. Ceramic period interest in the High Country reflects both the extensive amount of territory utilized during the Late Archaic as well as the emphasis on specific areas which coalesced during the Transitional period. The ceramic period numerical data ( 36 points at 20 sites) corresponds quite closely with that of the Late Archaic, while the two primary site clusters of the Ceramic period are in the same place as the two Transitional clusters.

Aspects of Subsistence and Settlement

The subsistence pattern in the High Country during prehistoric times is undoubtedly one of hunting and gathering. The artifact assemblage consists solely of those tools designed to obtain and process animals and wild plants, and the sites contain only those tool kits and features associated with these activities. Absent are the horticultural tools and architectural remains which characterize a horticultural subsistence component in other localities of the region.

The settlement patterns corresponding to a hunting and gathering 1 ifeway would either be a series of temporary camps occupied by a constantly moving small 
group, or a larger, more intensively occupied base camp from which hunting and gathering parties operated. It is extremely probable that the High Country was occupied during only part of the year; the winter season is inhospitable enough to suggest that only unusual pressures would force a group away from the more temperate lower elevations into the High Country during the winter months.

The general patterns of subsistence, settlement and seasonality can be applied to a11 five of the well-documented temporal periods. At the same time, each can be expected to exhibit their own variations of the patterns, resulting in the addition of a behavioral dimension to the temporal and spatial characteristics of each period. This serves to further distinguish and contrast each period with the others, within the overall framework of a small area and its consistent environmental attributes and limitations.

In attempting to assess the nature and degree of inter-period variations in subsistence and settlement, the question of assigning a site to a temporal period on the basis of diagnostic artifacts must be raised again. While no problem was encountered when single artifacts were employed to establish the extent of territorial utilization, a stronger case could be made for specific site occupation if two or more artifacts diagnostic of the same period were recovered there. The best case could obviously be made if multiple artifacts diagnostic of only one period were associated with a particular site. Although the number of sites with multiple artifacts diagnostic of a period is a much smaller sample than the total number of sites with diagnostic artifacts, their locations have already been shown to correspond to the focal points (or lack of them) characteristic of each period.

Two sites had multiple Late Paleo-Indian points associated: 41 CU 199 on the B Tue Ridge trail and 41 CU 255 on the Mescalero Campground trait (Fig. 2). Both sites are classified as Tithic scatters. Because 41 CU 199 also had Late Archaic and Ceramic period points present and four expanding stems from Late Archaic points were recovered at $41 \mathrm{CU} 255$, neither site was designated as a single component Late Paleo-Indian site. The two grinding slabs recovered at 47 CU 255 indicate vegetal processing activities occurred at this site in addition to hunting, although the assignment of these artifacts to one or another temporal period is not possible at this time. The presence of cutting and scraping tools and a relatively large number of debris flakes suggests that both sites were the scene of repetitive subsistence activities and may have served as regular campsites during successive seasonal exploitations of the High Country.

No sites are characterized by more than one point diagnostic of either the Early or Middle Archaic periods. The single identifiable Middle Archaic point was recovered from 41 CU 234 along with an Early Archaic point. Also recovered, however, were a Late Archaic point, a piece of historic glass and a 1918 cartridge casing. The site is a small lithic scatter, otherwise characterized by very few tools and a limited amount of flake debris. It seems to represent a temporary campsite used by small groups or even individuals at all periods of human occupation of the High Country. Its location in the Bowl (Fig. 2), along a current trail, further emphasizes the probability of constant, but light, visitation.

The six sites with more than one point diagnostic of the Late Archaic period are al1 characterized by the presence of midden features. This includes the one 
site (41 CU 244) which might be designated as single component, employing projectile point criteria only; it has three midden rings. The other five sites are characterized by midden rings, indeterminate middens or both. While midden rings are known from the Late Archaic period in central Texas (Weir 1976a), the most common type of burned rock feature by far is what is here referred to as the midden mound. In southeastern New Mexico and western Texas, however, the most common feature is the midden ring; its constant association with painted pottery types and radiocarbon dates ranging from A.D. 1100 to 1500 (Greer 1967) rather firmly relates this feature in the Guadalupe Mountains region to the Ceramic period, although Greer (1965:Fig. 3) illustrates Late Archaic and Transitional points which have been recovered from midden features.

As one of the characteristics of the Transitional period is the presumed introduction of the midden feature into the Park locality, it is not surprising to find that the three sites with multiple Transitional points are midden feature sites as well. A11 three $(41$ CU 215, 218, 221) are multiple and polytypic midden sites, having both midden rings and indeterminate middens present. A11 three are also situated at the head of South McKittrick Canyon (Fig. 2), two along the Mescalero Campground trail and the third in the canyon.

Five of the nine sites with either multiple Ceramic period points or painted pottery are also midden feature sites. Two of these five have one indeterminate midden only (41 CU 241 and 257); three are multiple midden sites, two of them having the relatively rare midden mound feature along with the midden ring (41 CU 231 and 258). 41 CU 231, moreover, would be considered as a single component Ceramic period site on the basis of projectile points alone. As three of the remaining sites are lithic scatters and the fourth is a rockshelter (41 CU 236), it would seem that the Ceramic period in the High Country is less characterized by the midden feature than are both the Late Archaic and Transitional periods.

Two alternative interpretations of the above data can be advanced: (1) midden features are diagnostic of the Ceramic period and hypothetically of the Transitional period also, and the presence of Late Archaic activity at sites with these features represents a prior use of the same location; or (2) midden features might be associated with any of the periods from Late Archaic through Ceramic. The weight of the evidence from surrounding localities in the region favors the former alternative. But it must be emphasized that, based on the evidence presently available in the High Country, either alternative might be acceptable.

By distinguishing between the various types of midden features, however, it is possible to suggest that the midden mound may have developed later in time than the midden ring. Although the sole single midden mound site (41 CU 210) had an Early Archaic point in association, all other mounds recorded are characterized by multiple Ceramic period points and painted pottery. While the feature is not very common, it most often appears at multiple midden sites in association with midden rings. As rings and mounds are thought to represent distinct types of features, the assumption can be made that mounds represent a subsistence activity different from, and supplemental to, that characterized by the midden ring.

The problem of the earliest introduction of the midden ring must be put aside, subject to a controlled excavation program. What is apparent from the preced- 
ing discussion is that the same locations were being exploited by different groups of people over a long period of time. Beginning in the Late Archaic period and continuing until the end of the Ceramic period and even later, three areas of the High Country exhibit increasing evidence of exploitation: the head and arms of Devils Den Canyon; the Mescalero Campground trail and the head of South McKittrick Canyon; and from the Bowl east along the Mckittrick Ridge Overlook (Figs. 1,2). Of the three, the Mescalero Campground trail appears to be the focal point of High Country activity; it is characterized by the largest absolute number of sites and by the largest number of those with multiple diagnostic artifacts indicating actual occupation.

Climatic data indicate that conditions became warmer and drier beginning in Late Archaic times (Gunn and Weir 1976:32). There would thus be a correlation between these changing climatic conditions, the expansion of grassland vegetative types into the High Country (including fiber plants), and the appearance and multiplication of midden features employed in the exploitation of fiber plants. Current vegetative maps (e.g., NPS 1976:32) indicate the grassland vegetation extends as far into the High Country as the Mescalero Campground trail area.

The picture which emerges from a study of High Country site and artifact distribution is one of continued hunting and increasing exploitation of vegetal products of the grassland type. Certain locations favor these products, due to a combination of altitude, drainage, sunlight and topography. Human exploitative requirements add the variables of open space, relatively flat terrain and access. All these factors serve to limit the possible locations favorable for subsistence activities, regardless of population size and energy needs. Most of the High Country sites are small and compact; larger sites do exist, however, in those areas where the terrain permitted the successful exploitation of subsistence activities over a wider area. These latter locations are the ones which show evidence of use during more than a single temporal period. Contrary to other opinions (e.g., Banks 1975, 1977), no differences seem to exist between upland and lowland sites with respect to subsistence, settlement, seasonality and presumably, therefore, group size and lifestyle.

\section{HIGH COUNTRY SITES CONSIDERED ELIGIBLE FOR LISTING IN THE NATIONAL REGISTER OF HISTORIC PLACES}

Based on the results of the 1976 survey and a review of previously recorded High Country sites, 16 of the 85 sites are considered eligible for listing in the National Register of Historic Places. This assessment is based on a comparative evaluation of all sites recorded in the Park, most of which have been personally visited by the writer. Several years of research into the prehistory of the region, moreover, provide an even broader base on which to make eligibility determinations.

A11 16 of the sites considered eligible for listing satisfy, in the writer's opinion, National Register Criterion (4), that portion of 36 CFR 800.10(a) which states that they" ". . have yielded, or may be likely to yield, information important in prehistory..." In that the National Register Criteria are "national." it is felt that more specific criteria should be developed and presented, criteria which pertain directly to this Park and the sites under con- 
sideration. It was this series of criteria, within the framework of National Register Criterion (4), which enabled the writer to recommend 16 out of 85 sites for eligibility. Table 19 provides descriptive and locational data for these 16 sites, and Table 20 indicates which of the criteria are applicable for each site.

The six criteria applied to all the sites in order to facilitate the selection process are the following:

1. Cultural Affiliation-the site can be associated with one or more temporal periods on the basis of multiple temporally diagnostic projectile points or pottery recovered within the site perimeter.

2. Unusual Feature(s)--the occurrence at the site of a unique feature or features which occur in low frequencies throughout the High Country as a whole. The midden mound would be an example of an "unusual feature."

3. Three or More Features--the presence at a site of at least three features, indicating extensive activity within a short period of time or repeated visits to the site over a longer period of time. "Feature" in this instance applies only to midden features, although the specific type is less important than the total number.

4. Feature Intersection--the occurrence at a site with multiple midden features of two or more of these features intersecting or overlapping. This indicates a periodicity of activity and presumably some time depth for the site.

5. Location--a site meeting other criteria which is situated on the periphery of the High Country area, or at least apart from the two major site clusters at the heads of Devils Den and South McKittrick Canyons. This criterion is intended to provide documentation of activities in these peripheral areas and of the territorial extent of occupation at certain periods.

6. Large Areal Extent--sites whose area exceeds 5000 square meters. The assumption is that large areas more likely indicate a long duration of occupation, multiple activities carried on concurrently, or a sizable group of people utilizing the site; any one of these interpretations is important for assessing the nature of High Country prehistoric behavior patterns.

None of the 16 sites considered eligible for listing satisfies all six of the above criteria of significance. All of the sites satisfy at least two criteria, however, and many have three or four of the criteria applicable (Table 20).

During the course of the survey, any evidence of disturbance at the sites was duly noted. All of the disturbance variables were studied and a classification established. Table 21 correlates a11 recorded High Country sites with these categories of disturbance.

Disturbances can be viewed along a continuum from actual to possible to unlikely; the agents of disturbance are either human or natural. A site located inside 


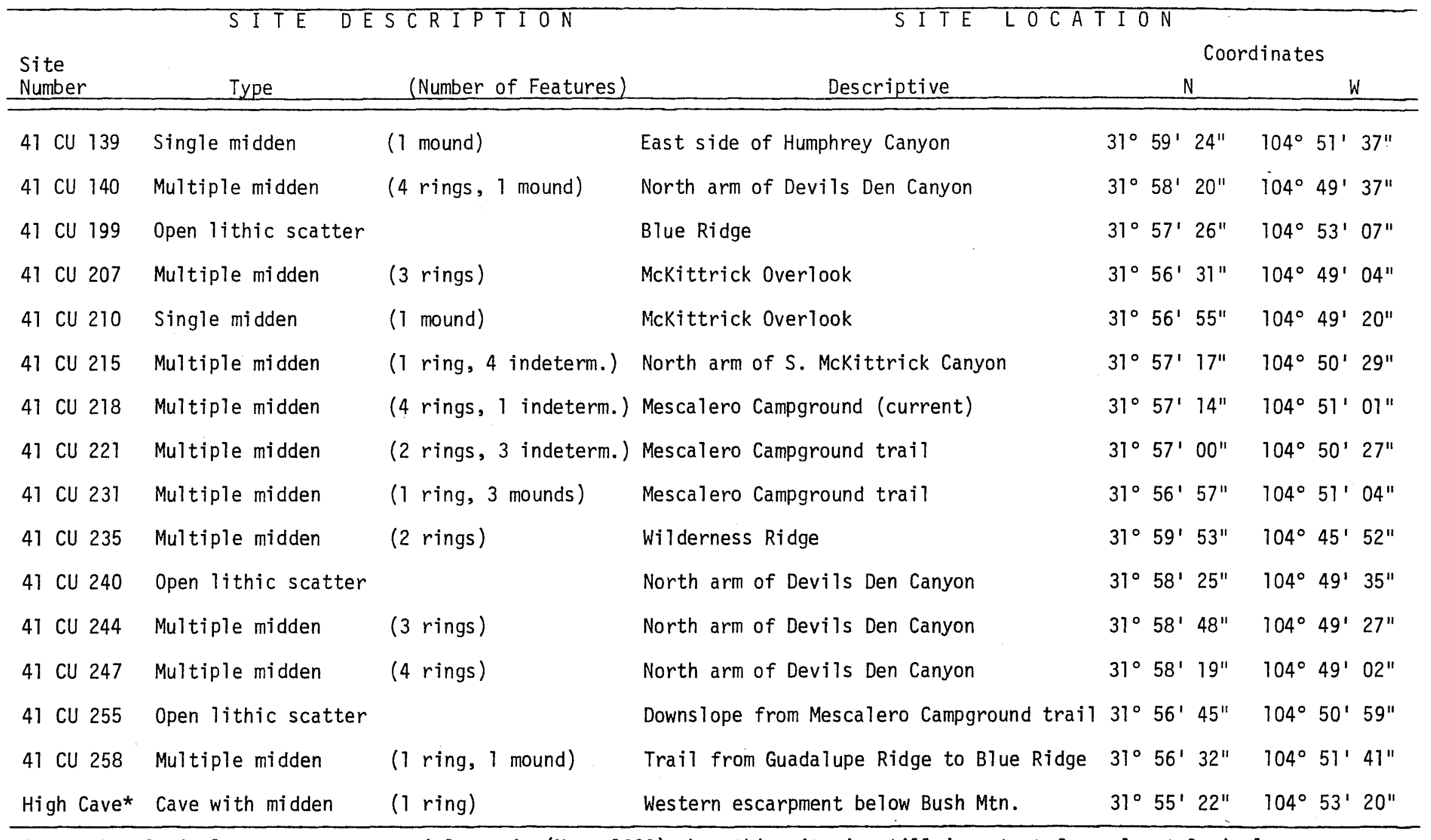

* No archaeological features or material remain (Mera 1938), but this site is still important for paleontological reasons. 
TABLE 20. SPECIFIC SITE IMPORTANCE FACTORS FOR DETERMINING NATIONAL REGISTER ELIGIBILITY

\begin{tabular}{|c|c|c|c|c|c|c|}
\hline $\begin{array}{l}\text { Site } \\
\text { Number }\end{array}$ & $\begin{array}{l}\text { Cultural } \\
\text { Affiliation } \\
\end{array}$ & $\begin{array}{l}\text { Unusual } \\
\text { Feature(s) }\end{array}$ & $\begin{array}{c}\text { Three or } \\
\text { more } \\
\text { Features } \\
\end{array}$ & $\begin{array}{l}\text { Feature } \\
\text { Intersection }\end{array}$ & Location & $\begin{array}{l}\text { Large } \\
\text { Area1 } \\
\text { Extent } \\
\end{array}$ \\
\hline 41 CU 139 & & $x$ & & & $x$ & \\
\hline 41 CU 140 & $x$ & & $x$ & $x$ & & $x$ \\
\hline 41 CU 199 & $x$ & & & & $x$ & $x$ \\
\hline 41 CU 207 & & $x$ & $x$ & $x$ & $x$ & \\
\hline \multicolumn{7}{|l|}{$41 \mathrm{CU} 210$} \\
\hline $41 \mathrm{CU} 215$ & $x$ & & $x$ & $x$ & & $x$ \\
\hline $41 \mathrm{CU} 218$ & $x$ & & $x$ & $x$ & & $x$ \\
\hline 41 CU 221 & $x$ & & $x$ & & & $x$ \\
\hline 41 CU 231 & $x$ & $x$ & $x$ & & & \\
\hline 41 CU 235 & $x$ & & & & $x$ & \\
\hline $41 \mathrm{CU} 240$ & $x$ & & & & & \\
\hline 41 CU 244 & $x$ & & $x$ & $x$ & & \\
\hline 41 CU 247 & & & $x$ & $x$ & & \\
\hline $41 \mathrm{CU} 255$ & $x$ & & & & & \\
\hline 41 CU 258 & $x$ & $x$ & & & & \\
\hline High Cave & & $x$ & & & $x$ & \\
\hline
\end{tabular}


TABLE 21. SITE DISTURBANCE VARIABLES AFFECTING ALL RECORDED HIGH COUNTRY SITES

\begin{tabular}{|c|c|c|c|c|c|c|c|c|c|c|c|c|c|c|}
\hline \multirow{14}{*}{$\begin{array}{l}\text { Sites } \\
(41 \mathrm{CU}---)\end{array}$} & \multirow{2}{*}{\multicolumn{2}{|c|}{$\begin{array}{ccc}\text { C A } & \text { M P I } \\
\begin{array}{c}\text { Designated } \\
\text { for }\end{array} & \begin{array}{c}\text { Designated } \\
\text { for Park }\end{array} \\
\text { Visitors } & \text { Personnel }\end{array}$}} & \multirow{2}{*}{$\begin{array}{l}N \quad G \\
\text { Unauthorized } \\
\text { (211) }\end{array}$} & \multirow{2}{*}{$\begin{array}{c}\begin{array}{c}\text { Passes } \\
\text { Through }\end{array} \\
* 139\end{array}$} & \multirow{2}{*}{$\begin{array}{c}T \\
\begin{array}{c}T \\
\text { Passes } \\
\text { By }\end{array} \\
\times(80)\end{array}$} & \multirow{2}{*}{$\begin{array}{l}A \\
P \text { a s } \\
30 \mathrm{~m} \\
\left(100^{\prime}\right) \\
175\end{array}$} & \multirow{2}{*}{$\begin{array}{c}\mathrm{L} \\
\mathrm{se} \mathrm{s} \\
60 \mathrm{~m} \\
\left(200^{\prime}\right) \\
\end{array}$} & \multirow{2}{*}{ 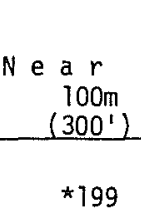 } & \multicolumn{2}{|c|}{$\begin{array}{l}\text { Evidence of } \\
\text { Erosion or } \\
\text { Sheetwash }\end{array}$} & \multirow{2}{*}{$\begin{array}{c}\begin{array}{c}\text { High } \\
\text { Visibility }\end{array} \\
118\end{array}$} & \multicolumn{3}{|c|}{$\begin{array}{c}\text { No } \\
\text { Foreseeable } \\
\text { Problem }\end{array}$} \\
\hline & & & & & & & & & 80 & 220 & & 23 & 201 & 242 \\
\hline & & 257 & [249] & (185) & (94) & 181 & 200 & 205 & 94 & 222 & 236 & 24 & 204 & 243 \\
\hline & & & & (186) & (184) & 206 & & (213) & 116 & 226 & & 117 & *207 & *244 \\
\hline & & & & 198 & 203 & (219) & & (230) & 184 & 230 & & 119 & 208 & 245 \\
\hline & & & & 202 & 217 & (226) & & $\star(255)$ & 185 & 232 & & 120 & 209 & 246 \\
\hline & & & & *221 & 224 & (249) & & & 186 & 249 & & 121 & $\star 210$ & $\star 247$ \\
\hline & & & & (222) & 225 & & & & 211 & 250 & & $* 140$ & 214 & 248 \\
\hline & & & & 227 & 228 & & & & 212 & 251 & & 169 & $\star 215$ & 253 \\
\hline & & & & ${ }^{+} 229$ & ${ }^{x}(232)$ & & & & 213 & $\star 255$ & & 176 & 276 & 256 \\
\hline & & & & *231 & 233 & & & & 219 & $\star 258$ & & 177 & 223 & *High Cave \\
\hline & & & & 234 & 254 & & & & & & & 180 & $\star 235$ & \\
\hline & & & & 239 & & & & & & & & 182 & $* 240$ & \\
\hline & & & & 252 & & & & & & & & 183 & 241 & \\
\hline
\end{tabular}

KEY TO SYMBOLS

* = Considered eligible for National Register listing

$x=$ Evidence of relic hunting observed

$+=$ Unused trail

$=$ Also Tisted in erosion category

[] = Listed in both trail and erosion categories 
a designated camping area (e.g., $41 \mathrm{CU} 218$ ) is subjected to actual disturbance by human agents, whereas a rockshelter may have only the potential of being disturbed by human agents if it is highly visible. Sites affected only by erosion or sheetwash, while disturbed, are not considered to be in as critical danger as those affected by human agents. Moreover, disturbance by natural agents is not sufficient cause to recommend mitigation measures, unless the site is of unusual significance or is also affected by human disturbance.

Four sites eligible for listing in the National Register of Historic Places are currently experiencing "adverse effects" as a direct result of visitor activity [36 CFR 800.9(a)]. $41 \mathrm{CU} 218$ is located within the perimeter of the Mescalero Campground; the Mescalero Campground trai1 passes through 41 CU 221 and 231; $41 \mathrm{CU} 139$ is crossed by the trail from Upper Dog Canyon to Humphrey Canyon (Figs. 2,7). In the case of $41 \mathrm{CU} 139$, it is recommended that the trail be shifted so as to avoid this site; as it is characterized by a single midden mound, trail realignment should satisfactorily mitigate the current adverse effect.

Sites 41 CU 218, 221 and 231 are characterized by multiple middens (Table 9), and the first two are among the largest in areal extent recorded in the High Country (Table 6). As the Mescalero Campground trail follows a ridge 1 ine, realignment would not seem to be practical. It is recommended that a program of archaeological testing be carried out at these three sites to recover the maximum possible amount of scientific information and to collect the surface artifacts. The midden features at these sites might then form the basis of an archaeological interpretive walk, thus capitalizing on their presence, proximity to the trail and the fact that, taken together, they encapsulate the entire prehistoric occupation of the High Country portion of Guadalupe Mountains National Park. 



\section{REFERENCES CITED}

Applegarth, S. M.

1976 Prehistoric Utilization of the Environment of the Eastern Slopes of the Guadalupe Mountains, Southeastern New Mexico. Doctoral dissertation, Department of Anthropology, University of Wisconsin. Madison.

Ayer, M. Y.

1936 The Archaeological and Faunal Material from Williams Cave, Guadalupe Mountains, Texas. Academy of Natural Sciences, Proceedings 88:599-619. Philadelphia.

Bailey, V.

1913 Life Zones and Crop Zones of New Mexico. North American Fauna 35. Washington.

Banks, K.

1975 Prehistoric Settlement in the Three-Mile and Sulphur Draw Watersheds. Report on file with the Soil Conservation Service, Temple and Department of Anthropology, Southern Methodist University, Dallas.

1977 Prehistoric Settlement in the Salt Basin, Texas. Transactions of the 12th Regional Archeological Symposium for Southeastern New Mexico and Western Texas:1-14. E1 Paso.

Beckett, P. H., M. C. Strong and S. M. Strong

1977 Nomad Trail Archaeological Project in Eddy County, New Mexico. Cultural Resources Management Division, Department of Sociology and Anthropology. New Mexico State University, Report 64. Las Cruces.

Clark, J.W., Jr.

1974 Rock Art of the Guadalupe Mountains National Park Area. Bulletin of the Texas Archeological Society 45:97-119.

Corley, J. A.

1965 Proposed Eastern Extension of the Jornada Branch of the Mogollon. Transactions of the First Regional Archeological Symposium for

Southeastern New Mexico and Western Texas:30-36. Hobbs, New Mexico.

Greer, J. W.

1965 A Typology of Midden Circles and Mescal Pits. Southwestern Lore 31:47-55. Boulder. 
1967 Some C-14 Dates from Midden Circle Sites. Manuscript provided courtesy of the author.

Griggs, $W$.

1973 A Survey of Historic Structures in Guadalupe Mountains National Park. Report on file with the National Park Service, Southwest Regional Office, Santa Fe and Department of Park Administration, Texas Tech University, Lubbock.

Gunn, J. and E. R. Prewitt

1975 Automatic Classification: Projectile Points from West Texas. Plains Anthropologist 20:139-149. Lincoln.

Gunn, J. and F. A. Weir

1976 Tool Kit Hypotheses: A Case of Numerical Induction. Newsletter of Lithic Technology V:37-39. San Antonio.

Hammersen, M. M.

1972 The Prehistoric Mogollon Culture and Its Regional Aspects in the E1 Paso Area. The Artifact 10(1):15-26. E1 Paso.

Henderson, $M$.

1976 An Archaeological Inventory of Brantley Reservoir, New Mexico. Department of Anthropology, Southern Methodist University, Contributions in Anthropology 18. Dallas.

Jelinek, A. J.

1967 A Prehistoric Sequence in the Middle Pecos Valley, New Mexico. Museum of Anthropology, University of Michigan, Anthropological Papers 31. Ann Arbor.

Katz, P.R. and S.R. Katz

1974 Preliminary Report of Activities of the 1974 Texas Tech University Archaeological Field School in Guadalupe Mountains National Park, Texas. Report on file with the National Park Service, Southwest Regional Office, Santa Fe and Department of Anthropology, Texas Tech University, Lubbock.

1975 A Summary of Recent Archaeological Investigations in Guadalupe Mountains National Park by the Department of Anthropology, Texas Tech University. Texas Archeology 19(2):11-15. Midland.

1977 Recent Investigations in the Southern Guadalupe Mountains Locality of West Texas. Transactions of the 12th Regional Symposium for Southeastern New Mexico and Western Texas:138-144. E1 Paso. 
Katz, P. R. and P. D. Lukowski

n.d. Results of Archaeological Survey in the Salt Flat Locality of Northern Hudspeth County, Texas. Report prepared for publication by the Natural Areas Survey, The University of Texas at Austin.

Katz, S. R. and P. R. Katz

1974 An Inventory and Interpretation of Prehistoric Resources in Guadalupe Mountains National Park, Texas. Report on file with National Park Service, Southwest Regional Office, Santa Fe and Department of Anthropology, Texas Tech University, Lubbock.

Lehmer, D. J.

1948 The Jornada Branch of the Mogollon. University of Arizona, Social Science Bulletin 17. Tucson.

1960 A Review of Trans-Pecos Texas Archeology. Bulletin of the Texas Archeological Society 29 (for 1958):109-144. Austin.

Levy, B.

1971 Guadalupe Mountains National Park: Historic Resource Study. Report prepared by Division of History, Office of Archeology and Historic Preservation, National Park Service. Washington.

Mera, H. P.

1938 Reconnaissance and Excavation in Southeastern New Mexico. American Anthropological Association, Memoir 51. Menasha.

National Park Service

1975 Natural Resources Plan for Guadalupe Mountains National Park. Report prepared by Guadalupe Mountains National Park staff and Southwest Regional Office of Natural Science. Santa Fe.

1976 Final Environmental Statement of the Proposed Master Plan for Guadalupe Mountains National Park, Texas. Prepared by the National Park Service. Washington.

Phelps, A. L.

1974 An Analysis of the Ceramics of the Guadalupe Mountains National Park. Bulletin of the Texas Archeological Society 45:121-150. Austin.

Schroeder, A. (ed.)

1965 Pratt Cave Studies, Guadalupe Mountains, Texas. Report on file with National Park Service, Southwest Regional Office, Santa Fe and Carlsbad Caverns/Guadalupe Mountains National Parks Headquarters, Carlsbad. 
Shafer, H. J.

1970 A Preliminary Report of an Archeological Survey in the Guadalupe Mountains National Park by the Texas Archeological Society in June 1970. Texas Archeology 14(3):10-17. Dallas.

Shelford, V. E.

1963 The Ecology of North America. University of Illinois Press, Urbana.

Suhm, D. A. and E. B. Jelks

1962 Handbook of Texas Archeology: Type Descriptions. Texas Archeological Society. Special Publication 1 and Texas Memorial Museum, Bulletin 4. Austin.

Taylor, W. W.

1966 Archaic Cultures Adjacent to the Northeastern Frontiers of Mesoamerica. In: Handbook of Middle American Indians, Volume 4: Archaeological Frontiers and External Connections (G. F. Ekholm and G. R. Willey, eds.):59-94. The University of Texas Press, Austin.

Warnock, B. H.

1974 Wildflowers of the Guadalupe Mountains and the Sand Dune Country, Texas. Sul Ross State University, Alpine.

Weir, F. A.

1976a The Central Texas Archaic. Doctoral dissertation, Department of Anthropology, Washington State University, Pullman.

1976b The Central Texas Archaic Reconsidered. In: The Texas Archaic: A Symposium (T. R. Hester, ed.):60-66. Center for Archaeological Research. The University of Texas at San Antonio, Special Report 2.

Whalen, M. E.

1977 Settlement Patterns of the Eastern Hueco Bolson. Centennial Museum, The University of Texas at El Paso, Anthropological Paper 4.

Willey, G. R. and P. Phillips

1958 Method and Theory in American Archaeology. University of Chicago Press. 


\section{APPENDIX I}

\section{CU 12}

At the request of Regional Archaeologist Ron Ice, a multiple midden site in the mouth of McKittrick Canyon was officially recorded as 41 CU 12. Ice first identified this site in conjunction with an on-site planning session concerned with the location of a parking area for McKittrick Canyon visitors. Although the parking area and $41 \mathrm{CU} 12$ overlap to some degree (Fig. 19), protective measures will be instituted so that no adverse effects will be incurred.

The site is located approximate1y 500 meters north of the Park boundary and 100 meters west of the proposed access road (Fig. 19,a). It is situated on a gentle terrace on the western edge of McKittrick Canyon and immediately north of the confluence of an intermittent stream and the canyon floor. It is bounded by the 5020-foot and 5040-foot contours; its dimensions are 150 meters north-south by 80 meters east-west for an area of 12,000 square meters.

Six prehistoric features were documented, all characterized by burned rock (Fig. 19,b). Two (Nos. 1 and 6) are complete ring middens; two (Nos. 3 and 5) may have functioned as ring middens but are actually crescents, with their horns oriented upslope; one (No. 2) seems to have been a midden mound, but, with a historic dugout constructed in its center, it is perhaps best to classify it as indeterminate; and one (No. 4) is a burned rock scatter.

Discussions concerning the function of burned rock features and the interrelationship of the various feature types may be found elsewhere in this report.

Table 22 summarizes the recorded data for each of the six prehistoric features, which were numbered according to the preliminary notation provided by Ice. 


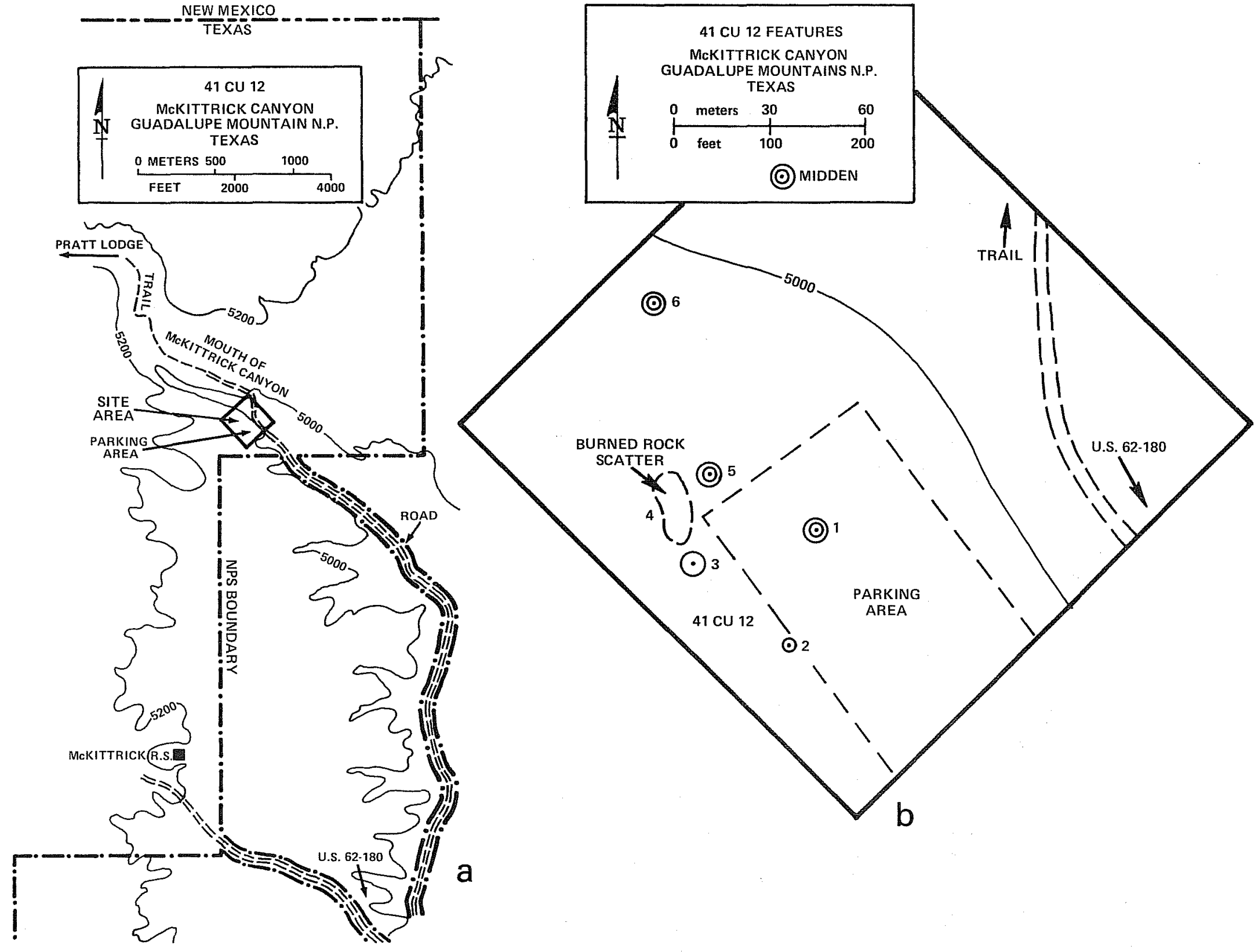

Figure 19. Site $41 \mathrm{Cu}$ 12. a, location; b, features. 
TABLE 22. 41 CU 12 FEATURE DATA

\begin{tabular}{|c|c|c|c|c|c|}
\hline $\begin{array}{l}\text { Feature } \\
\text { Number }\end{array}$ & $\begin{array}{l}\text { Feature } \\
\text { Type }\end{array}$ & $\begin{array}{c}\text { External } \\
\text { Diameter } \\
(\mathrm{m})\end{array}$ & $\begin{array}{l}\text { Height above } \\
\text { Ground Surface } \\
(\mathrm{m})\end{array}$ & Condition & $\begin{array}{l}\text { Associated } \\
\text { Artifacts }\end{array}$ \\
\hline 1 & Midden Ring & 9.0 & 1.2 & Fair to good & None \\
\hline 2 & $\begin{array}{l}\text { Midden Mound or } \\
\text { Indeterminate }\end{array}$ & 8.0 & 1.0 & $\begin{array}{l}\text { Poor; dugout } \\
\text { in center }\end{array}$ & $\begin{array}{l}\text { Historic } \\
\text { only }\end{array}$ \\
\hline 3 & Midden Crescent & 8.0 (est.) & 1.2 & Fair to good & None \\
\hline 4 & $\begin{array}{l}\text { Burned Rock } \\
\text { Scatter }\end{array}$ & $\begin{array}{l}\text { Indeter- } \\
\text { minate }\end{array}$ & None & Poor & None \\
\hline 5 & Midden Crescent & 10.0 (est.) & 1.2 & Good & None \\
\hline 6 & Midden Ring & 9.0 & 1.0 & Good & None \\
\hline
\end{tabular}





\section{APPENDIX II}

\section{CU 197}

Subsequent to our 1973 survey work in the Park, a number of individuals began reporting evidence of prehistoric occupation to us. These locations were visited whenever possible during the course of subsequent visits to the Park, and official recording procedures were carried out if appropriate. These informants consisted primarily of Park personnel and Texas Tech University researchers. One site, however, was reported to us by Mike Bilbo of El Paso, a member of the El Paso Rock Art Research Society engaged in an extensive pictograph documentation project in the region. Bilbo provided us with sketches of some of the pictographs, a brief report on the site itself and directions as to its location. We first visited the site in 1975 with the former Park Area Manager, John Chapman, and Susan Chapman, although its recording in the official Texas site numbering system was not accomplished until the summer of 1976.

The site consists of a shallow overhang formed in the east wall of Pine Springs Canyon immediately north of the fork in the stream bed where the Shumard Peak and Bush Mountain branches join. The overhang is situated about 10 meters above the stream bed and measures approximately 10 meters wide by 10 meters high. $A$ small cavity extending back into the vertical face was not explored for fear of disturbing the current resident.

While some ash was located on the shelf in front of the wall, just outside the roof line, no prehistoric artifacts were recovered. The only evidence of prehistoric activity at the site is the pictographs on the rear wall of the overhang.

While 20 individual pictographs were counted, only 10 were distinct enough for identification. One is a circle with eight lines radiating out from it, which may be a representation of the sun. There is one representation of a sheep, and there are eight identifiable human figures (Fig. 20). No color other than black was noted; there were no petroglyphs present.

The representational nature of the depictions at 41 CU 197 suggests closest correspondences with Park sites $41 \mathrm{CU} 24,41 \mathrm{CU} 48$ and 41 CU 70, rather than with the abstract depictions in Smith Cave (41 CU 15) (Clark 1974). As such, it most probably falls within the Jornada Style as defined by Schaafsma (1972), despite the presence of only monochrome depictions.

The absence of archaeological material at 41 CU 197 would correspond with a characteristic noted by Clark (1974:116) for other pictograph sites east of Guadalupe Peak:

Sites of the Jornada style consist of a wide range of motifs. Those on the east side of the Guadalupe Mountains massif consist of the least representational elements of the style and are not associated with archeological deposits. The styles on the west side of the massif and those in the Delaware Mountains consist of representational motifs and are associated with extensive midden deposits. The Jornada style paintings on the east side of the massif may have been ritualistic sites. 

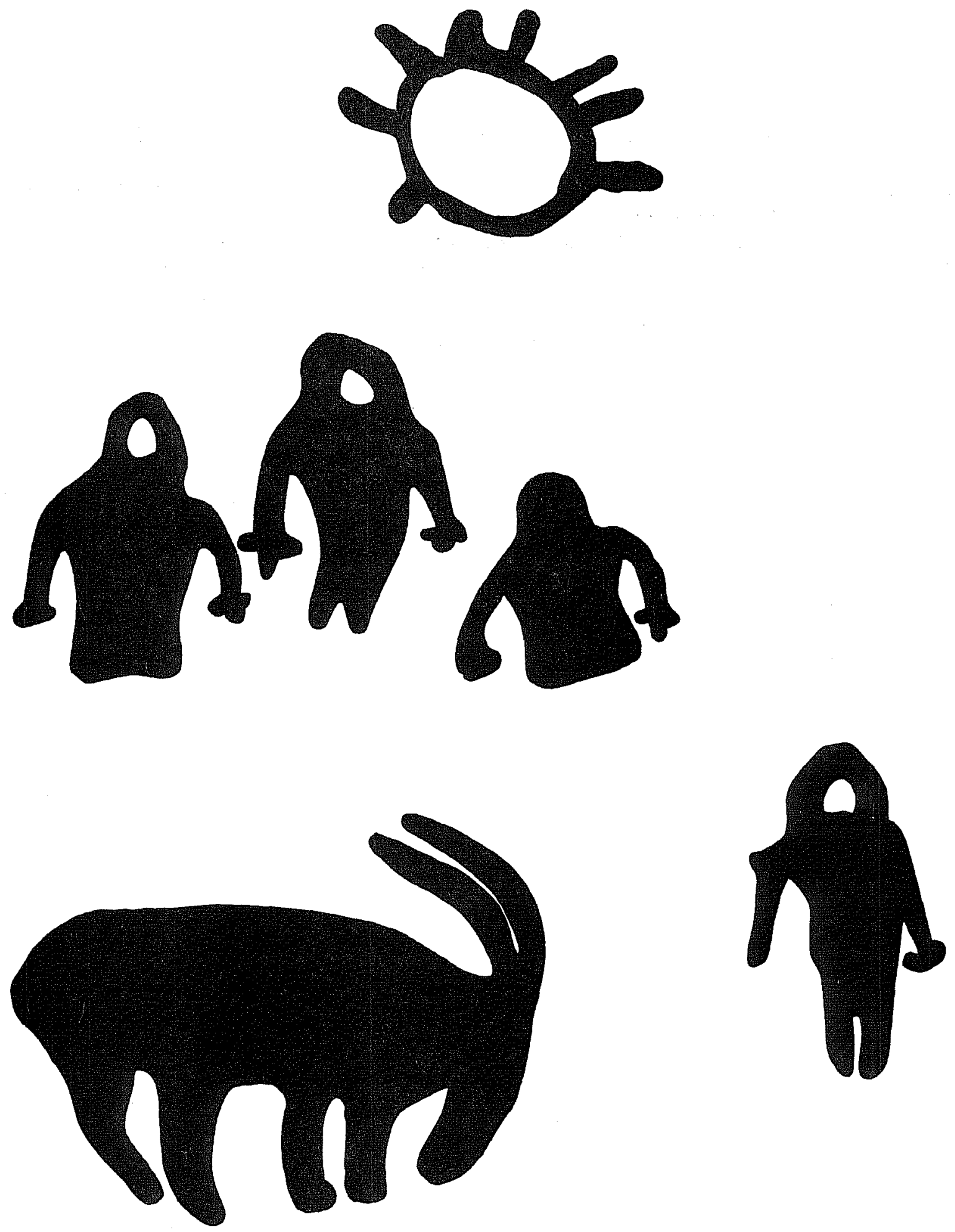

SELECTED PICTOGRAPHS AT 41 CU 197

Guadalupe Mountains, N.P., Texas

0

3

6

9

\section{Centimeters}

Figure 20. Selected Pictographs at 41 CU 197. 
The occurrence of a sheep depiction at 41 CU 197 has not yet been documented elsewhere in the Park, but this animal does appear at several localities at Hueco Tanks (Davis and Toness 1974).

In conclusion, the set of attributes at $41 \mathrm{CU} 197$, consisting of no artifacts and monochromatic representational depictions which include a sheep, makes this site unusual within the Park but certainly not unique within the region.

\section{REFERENCES CITED}

Clark, J. W., Jr.

1974 Rock Art of the Guadalupe Mountains, National Park Area. Bulletin of the Texas Archeological Society 45:97-119. Austin.

Davis, J. V. and K. S. Toness

1974 A Rock Art Inventory at Hueco Tanks State Park, Texas. El Paso Archaeological Society, Special Report 12.

Schaafsma, P.

1972 Rock Art in New Mexico. State Planning Office, Santa Fe. 
, 


\author{
APPENDIX III \\ TEST EXCAVATIONS AT 41 CU 97 \\ Susanna R. Katz
}

\title{
INTRODUCTION
}

Two features at site 41 CU 97 in Guadalupe Mountains National Park were tested during August 1976 at the request of Ron Ice, Regional Archaeologist, Southwest Region, National Park Service. These features would be adversely affected by access road improvements proposed for Upper Dog Canyon. Feature 5, a probable ring midden feature, was deemed close enough to the proposed road for heavy equipment to damage the feature; Feature 10, a burned rock concentration, would be completely destroyed by the road construction.

The site is $3 / 4$ mile in length, having a linear, north-south orientation paralleling the arroyo. Although the east side of the arroyo has a broader, flatter terrace than the west side, all the features and artifacts are situated on the west side of the arroyo. Sites $41 \mathrm{CU} 98,41 \mathrm{CU} 99$ and $41 \mathrm{CU} 198$ are also on the east side of the creek. Only one small collecting locality, CL-GP-5, has been located on the east side of the arroyo (Fig. 21,a).

Ten features are present7y associated with 41 CU 97; all but one of these are suspected to be ring middens. Similar features are known ethnographically as ovens for baking sotol (Dasilyrion leophyllum) and Agave sp. A light scattering of artifacts occurs over the entire site surface. As most of these artifacts represent cutting (Fig. 22,b), scraping (Fig. 22,a) and grinding tools (Fig. 16) which would have been used in conjunction with the ring middens, the primary function of the site is interpreted as the collection and preparation of vegetal matter.

It is our opinion that ring middens were constructed as close as possible to the source of the sotol. As sotol grows most profusely on well-drained slopes that receive a maximum amount of suniight, it would follow that the ring middens would be located on the west (i.e., east-facing slope) side of this north-south oriented, steep-sided canyon; although this side offers fewer flat and open areas in which to construct a ring midden, it has better conditions for the growth of sotol.

Information resulting from previous surveys (Shafer 1971; S. Katz and P. Katz 1974) and this excavation indicates that the earliest occupation of Upper Dog Canyon was during the Transitional Period (A.D. 1-800). The presence of expanding stemmed projectile points (Fig. 22,e) and other Transitional artifacts is, however, very rare. The major occupation appears to have been during the Ceramic Period (A.D. 800-1500), as evidenced by painted ceramics (Fig. 22,i) and small triangular arrow points (Fig. 22,c,d). It is assumed that most of the ring midden features also date to this period. There is some indication of a later Apachean occupation at the site, al though actual evidence for this is limited to two sherds from the surface $(F i g .22, g)$.

Sites 41 CU 98, 41 CU 99 and 41 CU 138 are also essentially Ceramic Period sites, suggesting that the canyon was utilized most intensively during this time. 

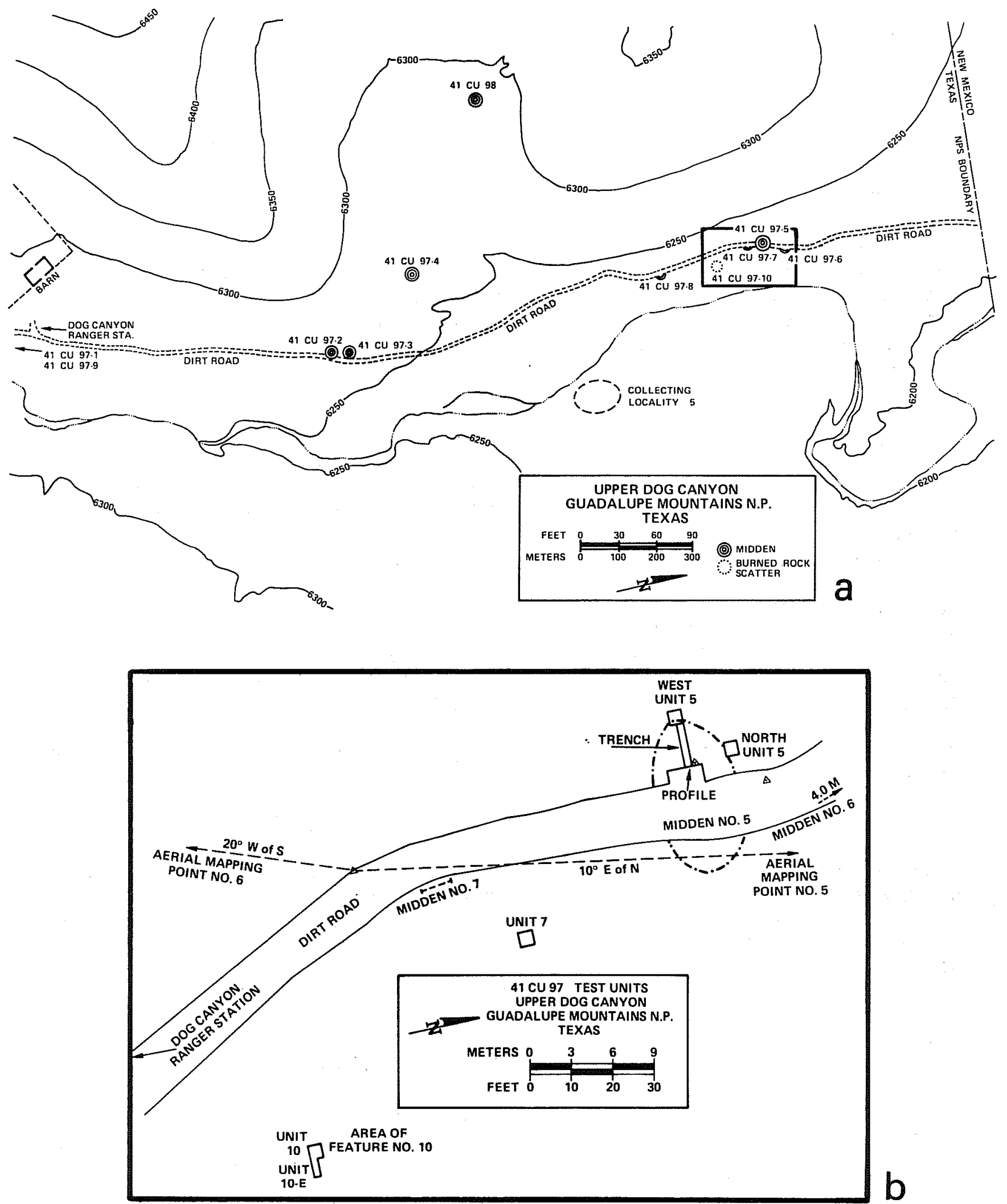

Figure 21. Site $41 \mathrm{cu}$ 97. a, location of site and features; b, excavation units. 


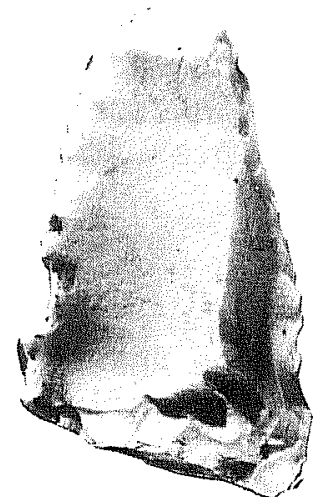

a

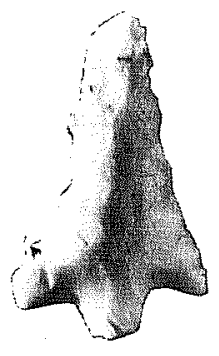

C

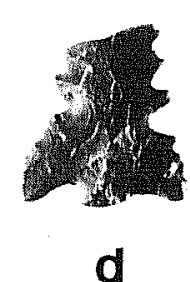

d

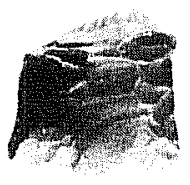

e

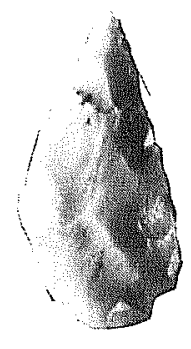

b
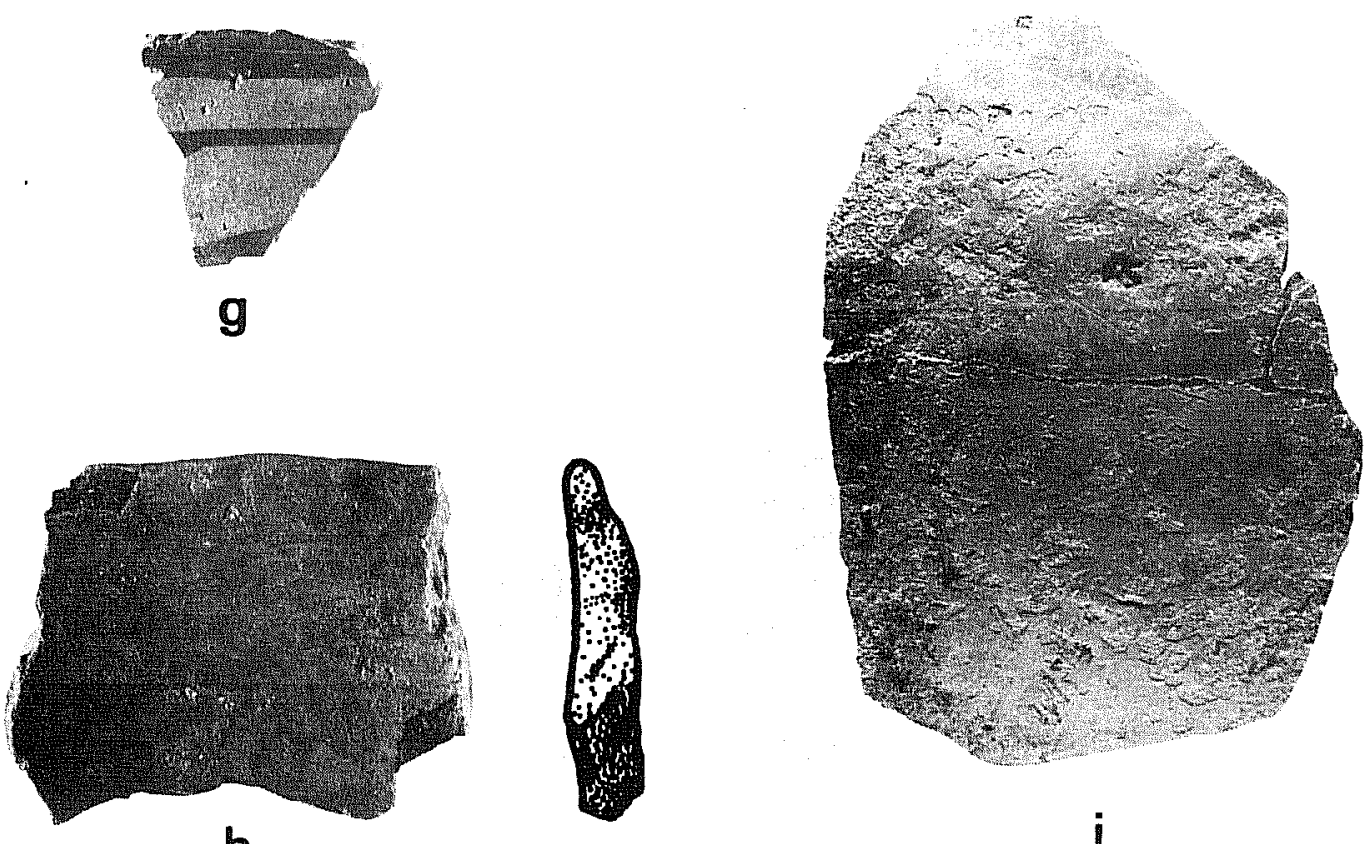

h

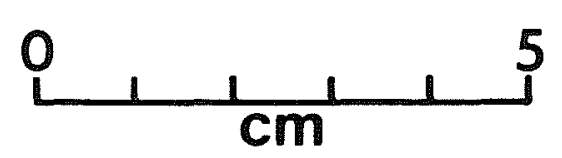

Figure 22. Selected Artifacts from $41 \mathrm{cl} 97$. a, side scraper; b, preform; $c$, Livermore point; d, Livermore point; e, expanding stem; $f$, rectangular or slightly contracting stem; g, painted body sherd; $h$, Jornada Brown rim sherd; i, El Paso Polychrome body sherd. 
Given that the four sites date to the same time period and that three are characterized by ring middens ( $41 \mathrm{CU} 138$ is a 1 ithic scatter only), we suggest that they may have formed an integrated activity complex.

\section{PREVIOUS ARCHAEOLOGICAL WORK}

The site was first recorded by a crew headed by Eugene Anderson during the Texas Archeological Society's 1970 Field School. At the time, five midden features were noted along the west side of the canyon. Feature 1 was a badly eroded midden on the west edge of the Upper Dog Canyon arroyo; Features 2, 3 and 5 had been cut by the road from the Texas-New Mexico state line to the ranger station and campground; Feature 4 was (and remains) the only fully intact feature at the site, located in a sloping meadow west of the access road (Fig. 21,a).

In 1973 the Katzes visited the site while conducting a prehistoric resource inventory of the Park, funded by the National Park Service through Texas Tech University (S. Katz and P. Katz 1974). Three additional features (numbered 6-8), all disturbed middens, were recorded at that time. These three had also been cut by the road, but the remains are located across the road from the middens recorded by Anderson; we suspect that the light and the time of day has a great deal to do with observing feature remnants ( $F i g .21, a)$.

During the 1976 High Country survey, funded by the National Park Service through The University of Texas at San Antonio, the Katzes recorded two more features. One was a partially eroded midden (Feature 9) located southwest of the Dog Canyon Ranger Station at the beginning of the Lost Peak Trail; the other, Feature 10, was a burned rock scatter east of the road on the side of a lateral arroyo, between the areas of Features 7 and 8 ( $F i g .21, a)$. The latter feature. (10) was first documented by Ron Ice in 1976, when he visited the canyon in conjunction with road planning.

\section{FEATURE 5 TESTING}

Feature 5 is a ring midden with the west side intact; most of the central depression and east side have been removed by construction of the present road and by natural erosion ( $F i g .21, b$ ). The intact (west) portion shows that the north-south dimension of the feature was at least 3 meters (Fig. 23,a); 2.7 meters remain of the east-west extent. A reconstruction of the midden would give a maximum east-west length of 6.75 meters (Fig. 21,b). Unlike the majority of ring middens found in this area, Feature 5 appears to have been asymmetrical, or at least not circular.

Exploratory work on the midden consisted of clearing a full north-south profile paralleling, but recessed from, the road (Fig. 23,b), and a $50-\mathrm{cm}$ wide east-west trench dug through the midden, bisecting the center of the north-south profile and extending to the end of the feature (Fig. 24,a). Two $1 \times 1$ meter units (5-west and 5-north) were placed at the surface extent of the burned limestone, both to locate the subsurface periphery of the feature and to observe construction details. This excavation plan allowed us to obtain the maximum amount of information on construction and use of the feature with the least amount of damage to its remaining section ( $F i g, 27, b)$. 


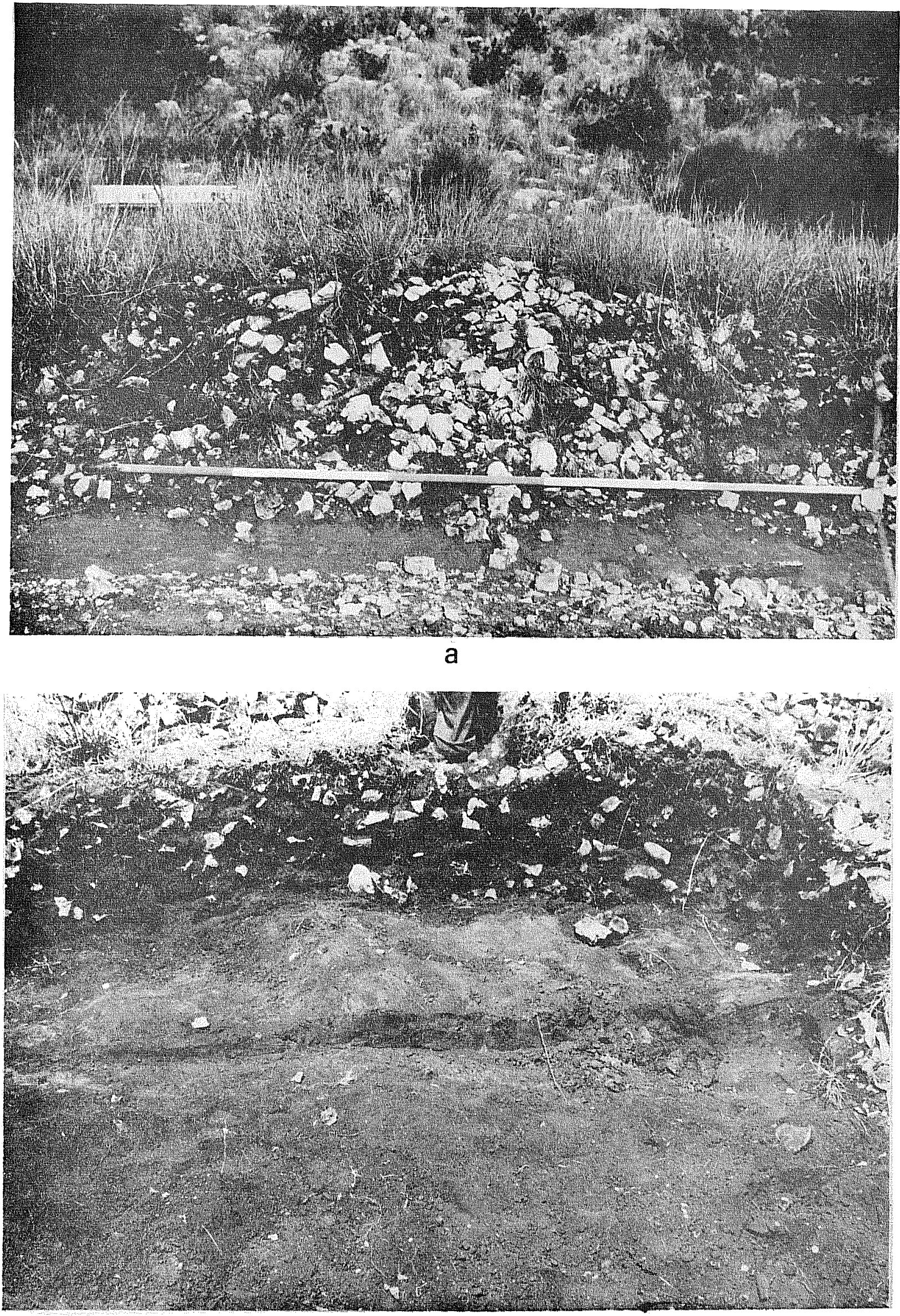

Figure 23. $41 \mathrm{Cu}$ 97, Midden \#5. Roadcut, looking west. a, before 


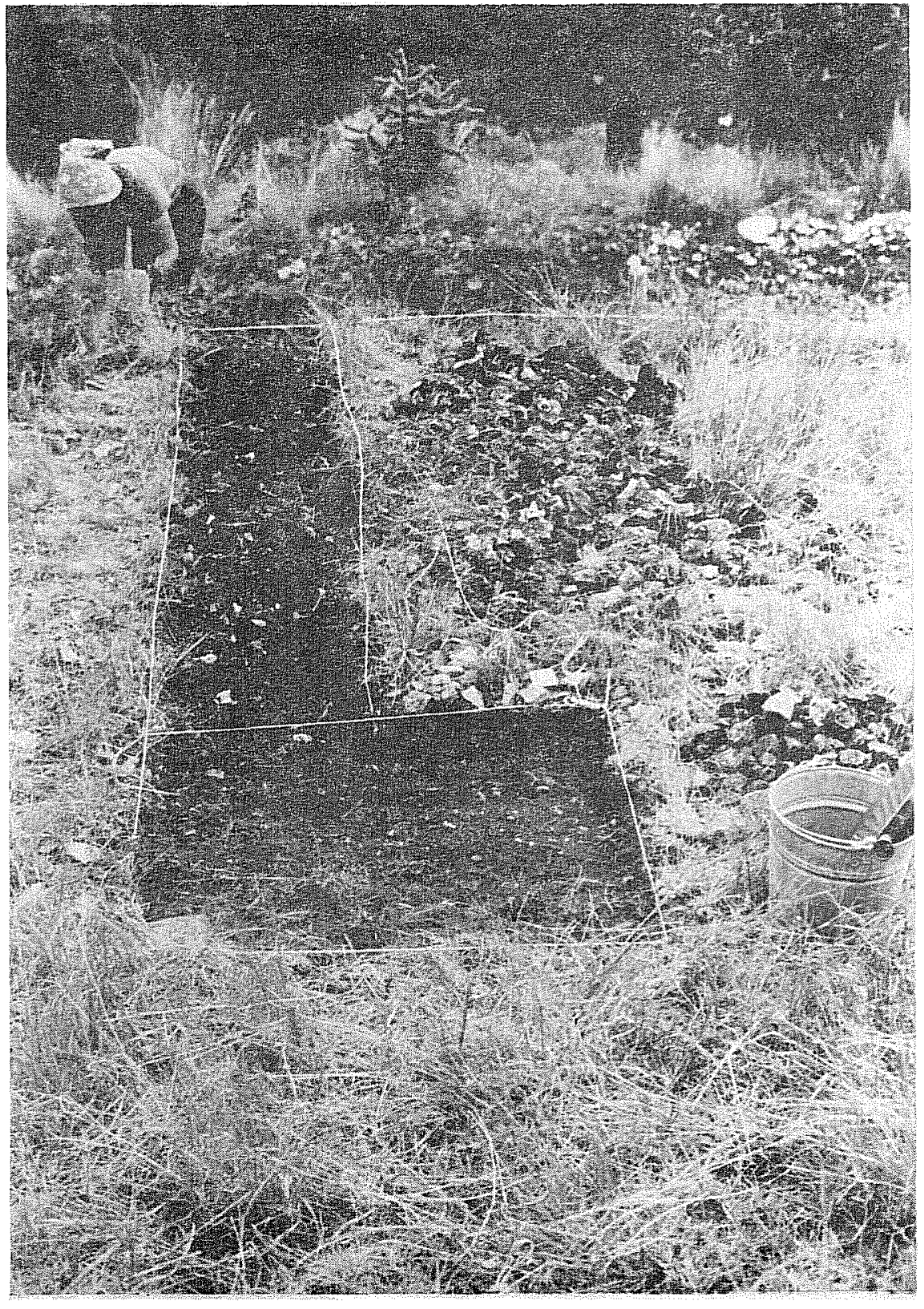

a

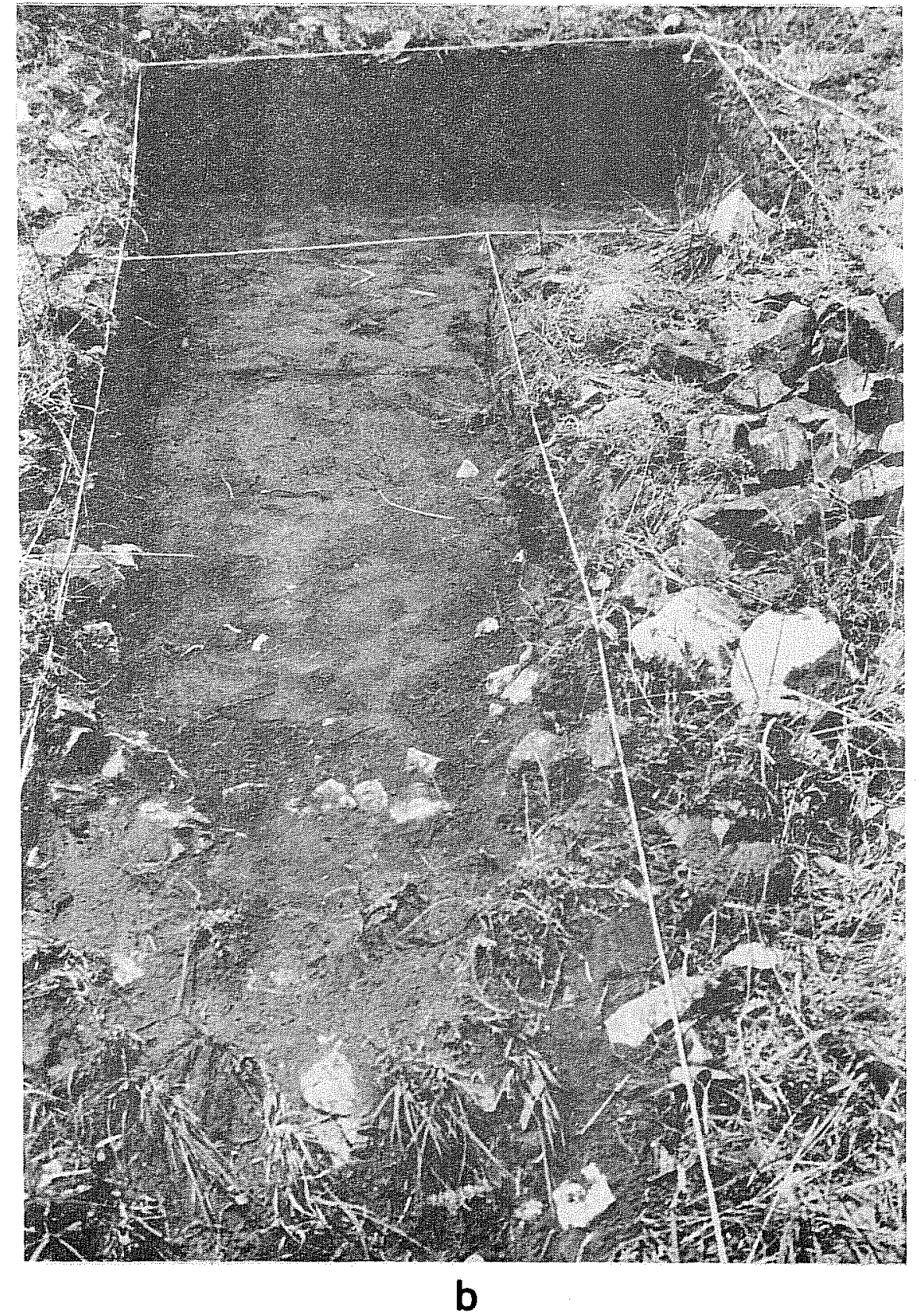

Figure 24. Selected Excavation Units at $41 \mathrm{cu} 97$. a, Midden \#5 trench and Unit 5-West, looking east; b, Units 10 and 10-East, looking west. 
The excavation indicated that the ring had been constructed directly on the existing ground surface, a red, sandy clay. No subsurface pit was present, although a clearing or slight "scooping-out" of the central portion was indicated by a slight inward slope noted in the profile of the trench (Fig. 25). A mound of red subsoil was observed at 150 to $180 \mathrm{~cm}$ in the east-west trench profile; this may be the soil that was removed from the center of the feature in the "scoopingout" or clearing procedure. A situation similar to this was noted in the middens excavated by the Texas Tech University Archaeological Field School in Pine Springs Canyon (P. Katz and S. Katz 1974, 1975).

Mixed in with the burned limestone and charcoal that forms the walls of the feature were occasional burned chert flakes, handstone fragments, projectile points and pottery. There was no apparent patterning to their distribution. There was also no indication of internal, ring wall stratigraphy (Fig. 25).

A charcoal sample taken from the interior of the feature was submitted to the Radiocarbon Laboratory at The University of Texas at Austin. The sample (TX2806) yielded a date of $710 \pm 50$ B.P. g or A.D. 1240. Similar features in Eddy County, New Mexico, have been dated at A.D. 1160-1490 (TX367-370) (Greer 1967). Although we believe that the ring midden is not restricted to this chronological period, the presence of El Paso Polychrome and Jornada Brown sherds (Fig. 22, $h, i$ ), as well as Perdiz and Livermore points from the midden, suggests that such a date will be likely for Feature 5. Other ring middens at 41 CU 97 are suspected of being from different time periods. Feature 3, for example, has an expanding stem from a projectile point (Fig. 22,e) and no pottery in association with it. The feature may date from the Transitional Period; ceramics identified as Apache (Fig. 22,g) ( $H$. Warren, personal communication) and found at the site suggest that other ring middens may be of a more recent date.

\section{INTER-FEATURE ACTIVITY AREAS}

One $1 \times 1$ meter test unit was excavated in an effort to locate a possible activity area (either processing or camping) in the vicinity of the midden. Designated Unit 7 because of its proximity to Feature 7, this square was placed nearer the creek bed and between Features 5 and 7 (Fig. 21,b).

The possibility of the presence of activity areas adjacent to middens has not been as thoroughly investigated in the past as it might have been; rather, archaeologists have focused their attention on the midden features themselves. As these features are often devoid of artifacts, the general opinion is that "ring middens aren't worth excavating." Excavations at 41 CU 78 by the Texas Tech University Archaeological Field School in Pine Springs Canyon did, however, locate a small activity area adjacent to one of these features. The ring itself contained no artifacts, but the activity area exhibited a heavy concentration of such materials.

Unit 7 was excavated to a depth of $40 \mathrm{~cm}$. There was a scraping tool on the surface and a flake and burned limestone in the first $10 \mathrm{~cm}$. The remainder of the unit was culturally sterile.

It is not surprising that no specific evidence of the activities which must have taken place peripheral to the midden rings was documented in Unit 7 . This unit 


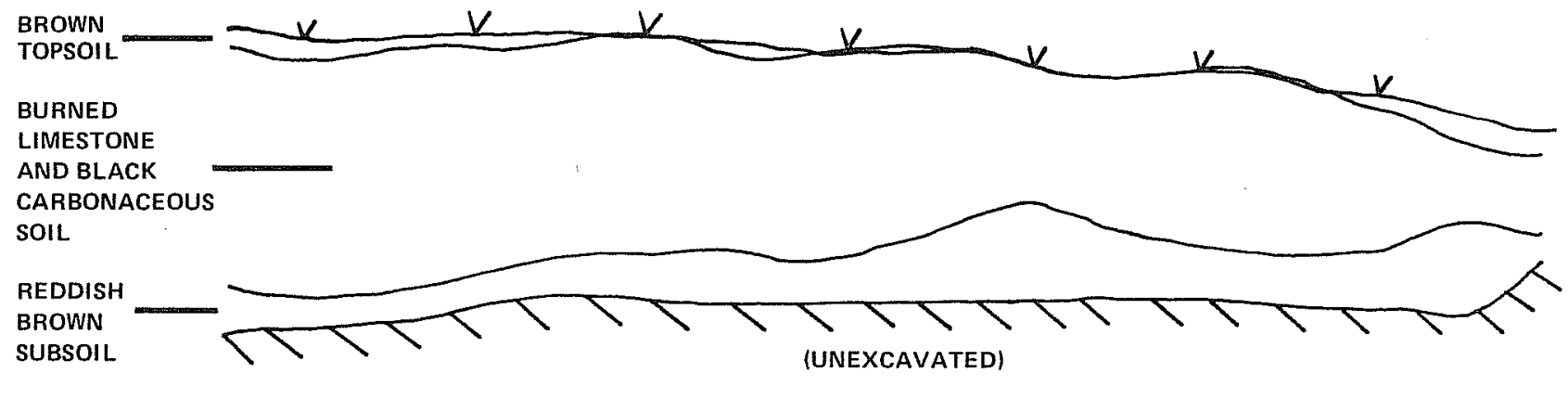

EAST-WEST TRENCH - SOUTH WALL

a

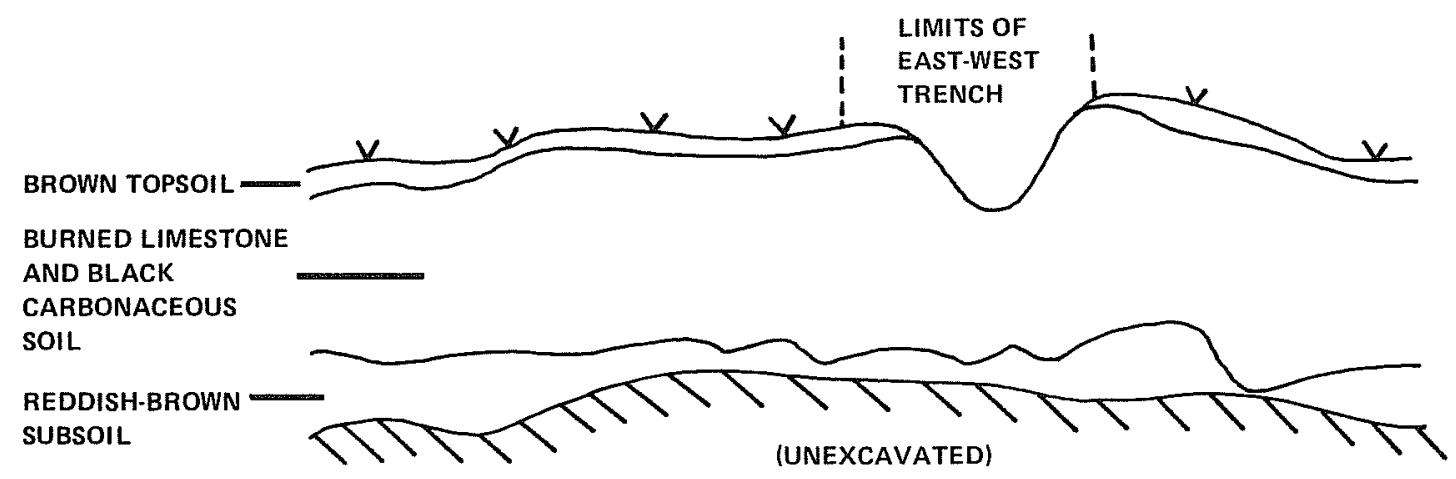

NORTH-SOUTH ROADCUT PROFILE - WEST WALL

41 CU 97 - MIDDEN NO. 5 PROFILES UPPER DOG CANYON GUADALUPE MOUNTAINS N.P. TEXAS

b

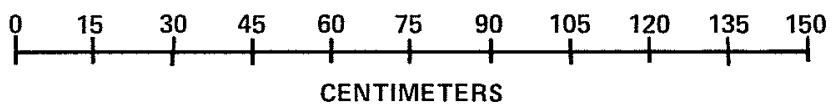

Figure 25. Selected Excavation Profiles at $41 \mathrm{cu} 97$, Midden \#5. a, south wall of east-west trench; b, west wall of north-south road cut profile. 
represents only one square meter of subsurface investigation. The light but consistent artifact scatter, extending almost $3 / 4$ mile, suggests that the entire area may have been suitable for supporting subsistence activities; no one particular spot need be used very heavily, or even more than once.

FEATURE 10 TESTING

Feature 10 is a burned rock concentration, 15 meters in diameter, located southeast of Feature 5 and close to the west bank of Upper Dog Canyon arroyo (Fig. 21,a). A smal1 lateral gully cuts through the feature.

The surface appearance of this feature resembles a midden mound, although the burned rock accumulation conformed to the slope of the ground and was not noticeably higher than the ground surface around it. There were several prom jectile points (Livermore and Perdiz) on the surface, along with El Paso Brown, El Paso Polychrome and Jornada Brown sherds, chert flakes and several handstones.

A trench was dug, bisecting the feature, and a $1 \times 1$ meter test unit was placed near the center of the concentration (Figs. 21,b; 24,b). The results indicated little activity below the single layer of burned limestone that formed the feature. Some ash and charcoal were noted to a depth of $10 \mathrm{~cm}$ below ground surface in the center of the concentration, but not elsewhere. Flakes were restricted to this upper level as well. This central unit was also in part destroyed by rodent activity.

Feature 10 may represent either a large hearth area or a ring midden in the early stages of construction and/or use. The lack of charcoal and ash anywhere but in the center of the feature, as well as the lack of a central pit or even a "scooped-out" central area, leads us to conclude that the area' was a large hearth rather than a seldom used ring midden.

\section{RECOMMENDATIONS}

Two of the ten known features at site 41 CU 97 have been tested. A sufficient amount of information has been gathered from them so that no further mitigation procedures are considered necessary with regard to construction of the improved road. Of the remaining features, we feel that Features 2, 3 and 4 cannot help but be adversely affected by the proposed development plan. Features 2 and 3 are proximate to a proposed parking area and 4 is in the campground area. Improvements to the road and facilities and increased visitor usage of the campground and environs will hasten the deterioration of these features.

It is recommended, therefore, that a further testing period be considered for Upper Dog Canyon. The testing period, of a proposed one week duration, would include an intensive surface collection of site $41 \mathrm{CU} 97$ and profiling of middens 2 and 3. Although these middens have been cut by the road, more than half of the original feature remains.

The information gained from this testing could result in an interpretive display similar to the botanical trail developed by Norman Stephan for Upper Dog Canyon. The trail could utilize the stabilized profiles of Features 2 and 3 to 
explain construction and use of the features and then lead to Feature 4, the intact midden. A final point on the trail could be site $41 \mathrm{CU} 98$, an extremely large ring midden 500 feet west of Feature 4 at site $41 \mathrm{CU} 97.41 \mathrm{CU} 98$ is considered eligible for listing in the National Register of Historic Places.

A side benefit of such an educational trail would be to eradicate the popular idea that ring middens are actually "treasure mounds" or storehouses of artifacts. By visually presenting the interiors of such features we feel that the point could be made that middens are essentially devoid of artifactual material yet played a critical role in the subsistence of previous inhabitants of this area.

\section{REFERENCES CITED}

Greer, J.W.

1967 Some C-14 Dates from Midden Circle Sites. Manuscript provided courtesy of the author.

Katz, P. R. and S. R. Katz

1974 Preliminary Report of Activities of the 1974 Texas Tech University Archaeological Field School in Guadalupe Mountains National Park, Texas. Manuscript on file with the National Park Service, Southwest Regional Office, Sante Fe and Department of Anthropology, Texas Tech University, Lubbock.

1975 A Summary of Recent Archaeological Investigations in Guadalupe Mountains National Park by the Department of Anthropology, Texas Tech University. Texas Archeology 19(2):11-15. Mid1and

Katz, S. R. and P. R. Katz

1974 An Inventory and Interpretation of Prehistoric Resources in Guadalupe Mountains National Park, Texas. Manuscript on file at National Park Service, Southwest Regional Office, Santa Fe and Department of Anthropology, Texas Tech University, Lubbock.

Shafer, H. J.

1971 A Preliminary Report of an Archaeological Survey in the Guadalupe Mountains National Park by the Texas Archeological Society in June, 1970. Texas Archeology 14(3):10-17. Dallas.

\section{ACKNOWLEDGMENTS}

Test excavations at 41 CU 97 in Upper Dog Canyon were conducted by members of the UTSA High Country field party. The north-south profile of Midden 5 was excavated and recorded by Susanna Katz, with the assistance of Bryan Aivazian; the latter also tested Feature 10. Paul Lukowski excavated Units 5-North and 7 and 
assisted with the field mapping of the excavation units. Paul Katz excavated Unit 5-West and was responsible for the field mapping. Ranger Harry Steed excavated the east-west trench connecting the Midden 5 profile with Unit 5-West; Anne Steed assisted with screening material recovered from the profile excavations. We are all especially grateful to Anne and Harry Steed for much iced tea and good company during our stay in Upper Dog Canyon.

\section{POSTSCRIPT}

Prior to our beginning work at $41 \mathrm{CU}$ 97, Regional Archaeologist Ron Ice provided us with a site analysis drawing indicating the proposed location of road and campground improvernents. Some of the midden features recorded in Upper Dog Canyon were drawn on this map and assigned working numbers. Table 23 below correlates these working numbers with those assigned to sites and features on file at the Texas Archeological Research Laboratory in Austin, Texas.

TABLE 23. CORRELATION OF SWRO WORKING NUMBERS AND OFFICIALLY RECORDED NUMBERS FOR SITES AND FEATURES IN UPPER DOG CANYON

Working Number

Midden \#1

Midden \#2

Midden \#3

Midden \#4

Midden \#5

Midden \#6

Midden \#7

Midden \#8
Official Designation

41 CU 97 -- Midden 5

41 CU 97 -- Feature 10

41 CU 97 -- Midden 8

Unrecorded

41 CU 97 -- Midden 3

41 CU 97 -- Midden 2

41 CU 97 -- Midden 4

41 CU 98 
